\author{
Aus der Abteilung Neurologie \\ (Prof. Dr. med. M. Bähr) \\ im Zentrum Neurologische Medizin \\ der Medizinischen Fakultät der Universität Göttingen
}

\title{
Der Effekt des Alterns auf funktionelle Deaktivierung im somatosensorischen System
}

\author{
der Medizinischen Fakultät \\ vorgelegt von \\ Jan Martin Sohns \\ aus \\ Göttingen
}

INAUGURAL - DISSERTATION

zur Erlangung des Doktorgrades

der Georg-August-Universität zu Göttingen

Göttingen 2009 
Dekan: Prof.Dr.med.C.Frömmel

I. Berichterstatter: Prof.Dr.med.A.Kastrup

II. Berichterstatter/in:

III. Berichterstatter/in:

Tag der mündlichen Prüfung: 


\section{Inhaltsverzeichnis}

$\begin{array}{ll}\text { 1. Einleitung } & 04\end{array}$

2. Material, Methode und Probanden 06

$\begin{array}{lll}2.1 & \text { Material } & 06\end{array}$

2.1.1 Grundlagen der fMRT basierend auf dem BOLD-Effekt 06

2.1.2 Repräsentation des sensorischen Kortex im fMRT 13

2.2 Probanden 14

2.3 Methode 16

2.3.1 Sensorische Stimulation mit $40 \mathrm{~Hz} \quad 16$

$\begin{array}{lll}2.3 .2 & \text { Finger-Tapping (FT) } 20\end{array}$

2.3.3 fMRT-Daten-Erfassung 21

2.3.4 fMRT-Daten-Analyse 22

2.3.5 Psychophysiologische Messungen (CPT) 24

2.3.5.1 Simultane Messungen 25

2.3.5.2 CPT-Testlauf 26

2.3.5.3 CPT-Messungen unter 40-Hz-Stimulation 27

3. Ergebnisse 29

3.1 Hämodynamische BOLD-Antwort beim FT 29

3.2 BOLD-Antworten der sensorischen Stimulation mit $40 \mathrm{~Hz} \quad 32$

3.2.1 Analyse des SI-Kortex 34

3.2.2 Analyse des SII-Kortex 36

3.2.3 Analyse weiterer Kortexareale 38

3.2.4 Gruppen-Analysen 41

3.2.5 Einzelprobanden-Analysen 43

3.2.6 Detaillierte Einzelbetrachtung der Probanden 46

3.3 Ergebnisse der CPT-Messungen 48

4. Diskussion $\quad 53$

5. Zusammenfassung $\quad 65$

$\begin{array}{ll}\text { 6. Literaturverzeichnis } & 67\end{array}$

$\begin{array}{ll}\text { 7. Abkürzungsverzeichnis } & 76\end{array}$ 


\section{1.) Einleitung}

Mit der Einführung der funktionellen Magnetresonanztomographie (fMRT) im vergangenen Jahrzehnt haben die Neurowissenschaften einen enormen Wandel erfahren. Die meisten fMRT-Techniken basieren auf dem Kontrast des BOLD-Effekts (BOLD: Blood ㅇxygenation Level Dependent) mit der Benutzung von paramagnetischem Desoxyhämoglobin (desoxy-Hb) als endogenem KontrastVermittler (Bandettini et al. 1992, Ogawa et al. 1992). Diese Methode ist eine der bedeutendsten geworden, um menschliche Gehirn-Areale funktionell darzustellen (Ogawa et al. 1990). Die fMRT-Technik ist sehr gut geeignet für nicht-invasive Messungen der menschlichen Gehirn-Aktivität und für die Bestimmung von deren Lokalisation in nahezu Echtzeit. Sie ist damit die ideale Methode zum Vergleich der Gehirn-Aktivität von unterschiedlichen Altersgruppen bei einem bestimmten Stimulus. Die Entwicklung der fMRT-Technik, basierend auf dem BOLD-Effekt, bietet besonders in der Neurologie, Psychiatrie und Neurochirurgie neue Möglichkeiten. Etabliert sind diese Techniken zur Lokalisation von aktivierten Gehirnarealen bereits im Bereich der neurokognitiven Forschung. Die BOLD-Technik wird aber auch zunehmend für die Darstellung von Reorganisationsprozessen nach zerebralen Infarkten oder für die prächirurgische Lokalisationsdiagnostik sensomotorischer und sprachlicher Funktionen bei Patienten mit Epilepsien, vaskulären Malformationen und Tumoren eingesetzt. Mit dem Einsatz dieser Methoden ist somit, neben neuen Erkenntnissen über den funktionellen Aufbau des Kortex und Ablauf des Alterungsprozesses, die Hoffnung auf eine verbesserte Patientenversorgung verbunden. Strukturen kortikaler Aktivierung und Deaktivierung hängen vom Alter eines Probanden ab, denn Altern ist verbunden mit strukturellen, neurochemischen, anatomischen, physiologischen und metabolischen Veränderungen im menschlichen ZNS (Kuhl et al. 1982, Leenders et al. 1990, Marchal et al. 1992). Das Wissen über physiologische Alterungsprozesse des somatosensorischen Kortex ist relativ gering und zum Teil widersprüchlich. Eine große Gruppe von neurologischen Defiziten tritt in den höheren Lebensdekaden (60-80 Jahren) auf und verursacht motorische Defizite. Dazu zählen Schäden zerebrovaskulärer oder neurodegenerativer Genese. Die Pathophysiologie ist nicht völlig erforscht und deswegen unumgänglicher Gegenstand intensiver Studien. Um pathophysiologische Prozesse besser verstehen 
zu können, ist es notwendig, erst einmal, wie in unserer Studie geschehen, das normal alternde menschliche ZNS an gesunden jüngeren und älteren Probanden zu untersuchen und physiologische Prozesse nachzuvollziehen (Sailer et al. 2000). Es ist allgemein anerkannt, dass positive BOLD-Antworten (PBR: Positive B $O L D$ Response) einen Indikator für Anstiege von neuronaler Aktivität im Gehirn darstellen. Neben dem Auftreten von positiven BOLD-Signal-Antworten wurden in mehreren Studien ebenfalls negative BOLD-Signal-Antworten (NBR: Negative B Response) beobachtet, so zum Beispiel im okzipitalen Kortex während verschiedener visueller Aufgaben und Stimulationen (Chen et al. 2005, Shmuel et al. 2002, Smith AT et al. 2004). Neben dem visuellen und motorischen Kortex wurde das Phänomen der NBR kürzlich im somatosensiblen System während taktiler und elektrischer Stimulation beobachtet (Arthurs et al. 2004, Hlushchuk und Hari 2006). Darüberhinaus wurde in jüngster Vergangenheit durch simultane fMRT- und elektrophysiologische Aufnahmen in Tierexperimenten deutlich gezeigt, dass es eine enge Korrelation zwischen NBR und lokalen Abfällen in neuronalen Aktivitäten gibt. Die Gruppe um Shmuel et al. (2006) zeigte diese Beobachtung zum Beispiel innerhalb des visuellen Kortex beim Affen. Doch wie genau verhält sich diese Korrelation zwischen NBR und Inhibition beim Menschen und wie verändert sich dieser Einfluss im höheren Lebensalter? Ziel dieser Studie ist die Bestimmung der funktionellen Bedeutung und Korrelation von ipsilateralen NBR und neuronaler Inhibition während unilateraler sensorischer Stimulation unter dem Einfluss des Alterns. Weil das somatosensible System die Möglichkeit der quantitativen Messung von sensorischen Funktionen bietet (Blankenburg et al. 2003, Davey et al. 2001, Yamashita et al. 2002), wurden von uns BOLD-Antworten während gleichzeitiger unilateraler Stimulation des rechten N. medianus gemessen. Direkte N. medianusStimulationen zeigten in vergangenen Studien eine deutliche Aktivierung des sensomotorischen Kortex (Davis et al. 1995, Baudewig et al. 1997, Büchert et al. 1997). Zusätzlich bestimmten wir gleichzeitig die Wahrnehmungs-Reizschwelle (CPT: urrent Perception Ihreshold) im linken Zeigefinger der Gegenseite als eine quantitative Messung der sensorischen Funktion durch elektrische Stimulation, um eine mögliche Korrelation von ipsilateralen NBR und sensorischer Inhibition konkret darstellen zu können. Die Abklärung der Entstehung sowie die funktionelle Bedeutung von länger anhaltenden NBR sind grundlegend für das Verständnis der Zusammenhänge von neuronalen Schaltwegen im Kortex, die sich im höheren 
Lebensalter verändern können, und bieten damit eine gute Betrachtungsweise für den Effekt des Alterns auf funktionelle Deaktivierung im somatosensorischen System.

\section{2.) Material, Methode und Probanden}

\subsection{Material}

\subsubsection{Grundlagen der fMRT basierend auf dem BOLD-Effekt}

Die Messungen unserer Studie wurden mittels fMRT durchgeführt. Dies ist eine Technik, die intrinsische Änderungen der Oxygenierung des Bluts im menschlichen Gehirn mit magnetischer Resonanz darstellt. In den letzten Jahren ist diese Technik der Bildgebung zu einer der erfolgreichsten Werkzeuge geworden, Aktivierungen des menschlichen Gehirns nicht-invasiv zu untersuchen. Das desoxygenierte Hämoglobin ist in einem paramagnetischem Zustand. Wird es dagegen oxygeniert, geht es in einen diamagnetischen Zustand über. Wie der Kontrast bei einer MRTAufnahme einer Blut-Probe ausfällt, ist von der Konzentration des desoxy-Hb abhängig. Je mehr desoxy-Hb sich in der Probe befindet, desto stärker interferiert das Magnetfeld des desoxy-Hb mit dem des MRT-Scanners und die $\mathrm{T}_{2^{-}}$ Relaxationszeit wird verändert (Thulborn et al. 1982). Dieser Effekt ist bekannt als BOLD-Effekt. Im gesunden und gut funktionierenden Gewebe wird dieser Effekt neben dem Blut in den Gefäßen ebenfalls durch das Zellgewebe um die Gefäße herum verursacht, wie es bei Ogawa et al. (1990) sowie Ogawa und Lee (1990) genauer beschrieben wird. Der BOLD-Effekt ist damit ein sehr guter Parameter um nachzuweisen, wo sich gerade besonders viel oder wenig desoxygeniertes oder oxygeniertes Blut zu einem bestimmten Zeitpunkt befindet. Die Konzentration von oxygeniertem, beziehungsweise von desoxygeniertem Blut zeigt an, ob bestimmte Kortexareale gerade aktiviert oder deaktiviert werden. Kurz gesagt ist der BOLDEffekt ein „endogenes Kontrastmittel“. Physiologischerweise steigt die Konzentration von desoxy- $\mathrm{Hb}$ im Normalfall vom arteriellen zum venösen Gefäßnetz um bis zu $40 \%$ an. Bei gleichzeitig durchgeführten fMRT-Aufnahmen führt die Zunahme des 
desoxy-Hb im Normalfall zu einer Abnahme des BOLD-Signals um bis zu 8 \%. Mit Zunahme der Aktivität im ZNS steigt allerdings die Intensität des BOLD-Signals, obwohl man eigentlich von einer erhöhten Aktivität mit einem größeren Sauerstoffverbrauch und einer Zunahme von desoxy-Hb, wie es aus der Körperperipherie und Diffusion am Kapillarbett bekannt ist, ausgehen müsste. Trotz eines stärkeren Sauerstoffverbrauchs und eines vermeintlichen Anstiegs des desoxy-Hb kommt es allerdings zu einer BOLD-Signal-Zunahme (Kwong et al. 1992). Dieses Phänomen liegt daran, dass sich die Konzentration von desoxy-Hb aus einem biophysikalischen und physiologischen Anteil zusammensetzt. Findet eine Aktivierung im Kortex statt, so steigt konsequenterweise der Sauerstoffverbrauch an und das von Stoffwechselprozessen gebildete desoxy-Hb verringert nun das MRT-Signal. Bei ZNS-Aktivitäten sinkt aber zur gleichen Zeit die Sauerstoffextraktionsfraktion und damit die Menge des angebotenen Sauerstoffs im Blut, die durch Diffusion von den Zellen entnommen wird. Die Abnahme der Sauerstoffextraktionsfraktion und der Anstieg des absoluten desoxy-Hb sind nur möglich, wenn gleichzeitig der lokale CBF überproportional und mit ihm das CBV ansteigt. Zusammenfassend erklärt sich der Prozess folgendermaßen: Wenn von Zellen des ZNS unter neuronaler Aktivität mehr Sauerstoff verbraucht wird, steigt dadurch die absolute Konzentration des desoxy-Hb zwar an, gleichzeitig steigt in größerem Maße aber auch der Blutfluss und die „relative“ Konzentration des desoxy$\mathrm{Hb}$ nimmt ab. Somit kommt es zu keinem Abfall des BOLD-Signals. Das desoxy-Hb erfährt in der Masse des zugeführten oxygenierten Bluts eine relative Abnahme und damit seinen verringernden Effekt auf das BOLD-Signal, obwohl das desoxy-Hb in absoluten Werten zunimmt. Das BOLD-Signal steigt also auf Grund des gesteigerten CBF bei neuronaler Aktivität an, fällt allerdings ab, bzw. bleibt gleich, wenn deaktiviert wird oder der CBF nicht steigt (Logothetis und Wandell 2004). In den Abbildungen 01 und 02 sind die oben aufgeführten Abläufe des BOLD-Effekts bildlich dargestellt. 


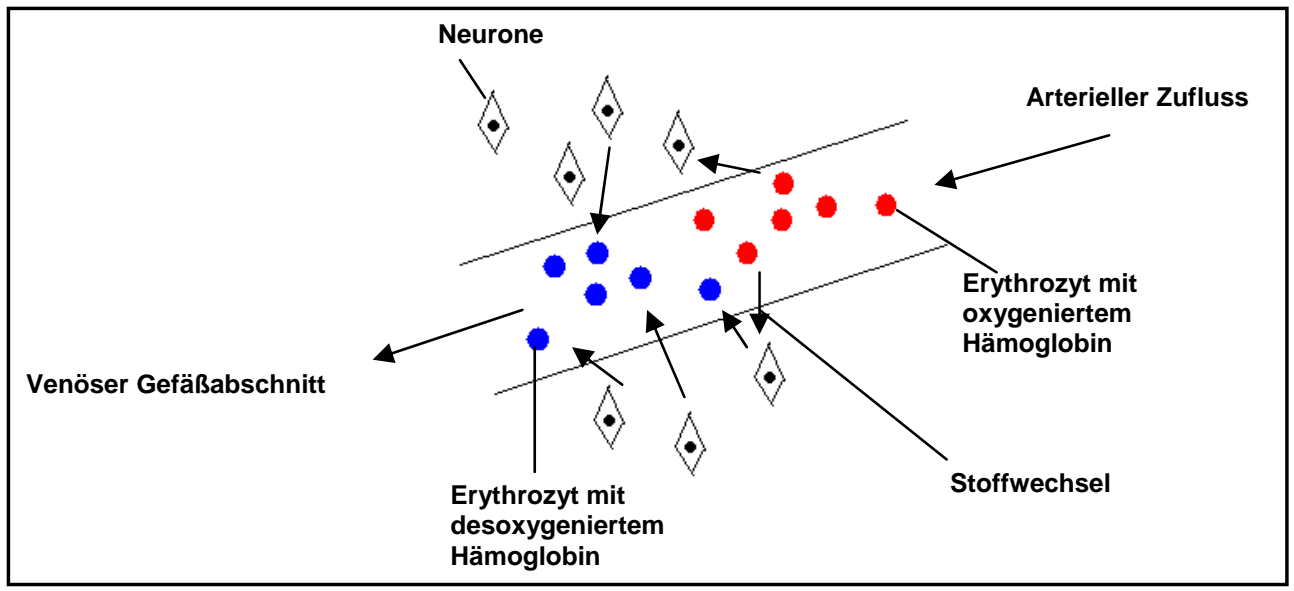

Abbildung 01: Ausgangszustand.

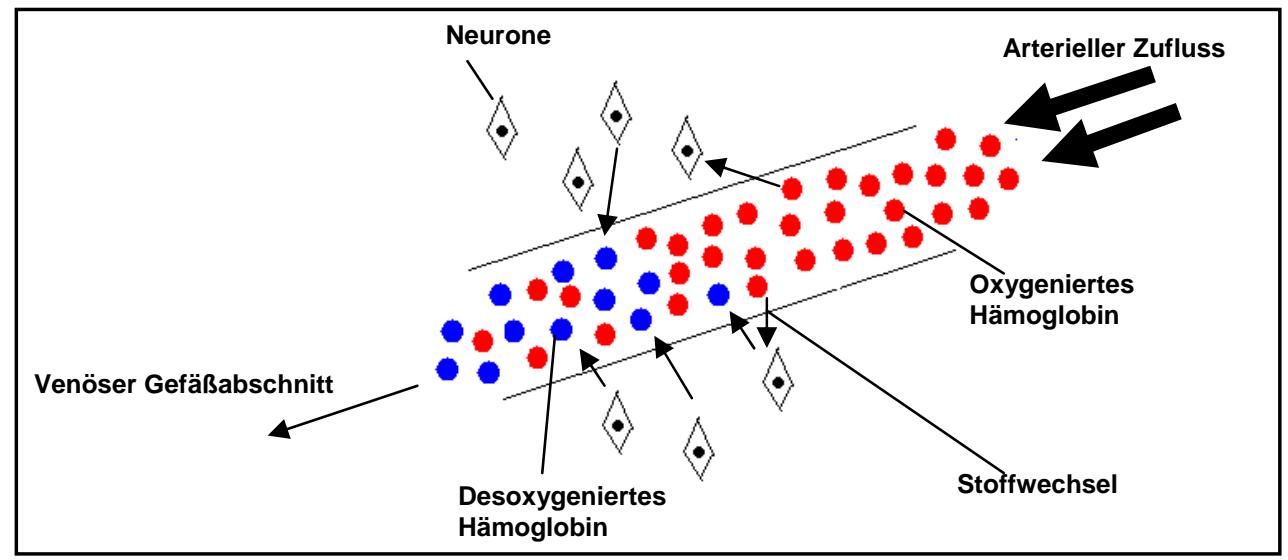

Abbildung 02: BOLD-Effekt, mit aktiviertem Kortexareal.

Um Strukturen und Funktionen einzelner Organe zu untersuchen, dienten am Ende des 19. und zum Anfang des 20. Jahrhunderts histologische Untersuchungen und Präparate, um physiologische und pathologische Prozesse des ZNS besser verstehen zu können. CT und MRT gab es noch nicht und Röntgenaufnahmen entwickelten sich noch. K. Brodmann klassifizierte 1909 die gesamte Großhirnrinde systematisch nach histologischen Gesichtspunkten und teilte sie in die heute bekannten Brodmann-Areale ein (Brodmann 1909). Damals war in den Neurowissenschaften relativ wenig über Aufgaben einzelner Hirnanteile bekannt, so dass es schwierig war, Funktionen und Aufgaben auf ein bestimmtes ZNS-Areal zu lokalisieren. Mit dem Fortschreiten von Wissenschaft und Untersuchungs-Techniken war es später möglich nachzuweisen, dass viele histologische Brodmann-Areale mit funktionellen Einheiten übereinstimmten und damit eine Grundlage zur Kartierung des menschlichen ZNS gelegt worden war. Brodmanns Einteilungen sind bis heute 
gültig und zeigen den engen Zusammenhang zwischen histologischen Strukturen und deren funktionelle Zusammenhänge und Aufgaben (Trepel 1999).

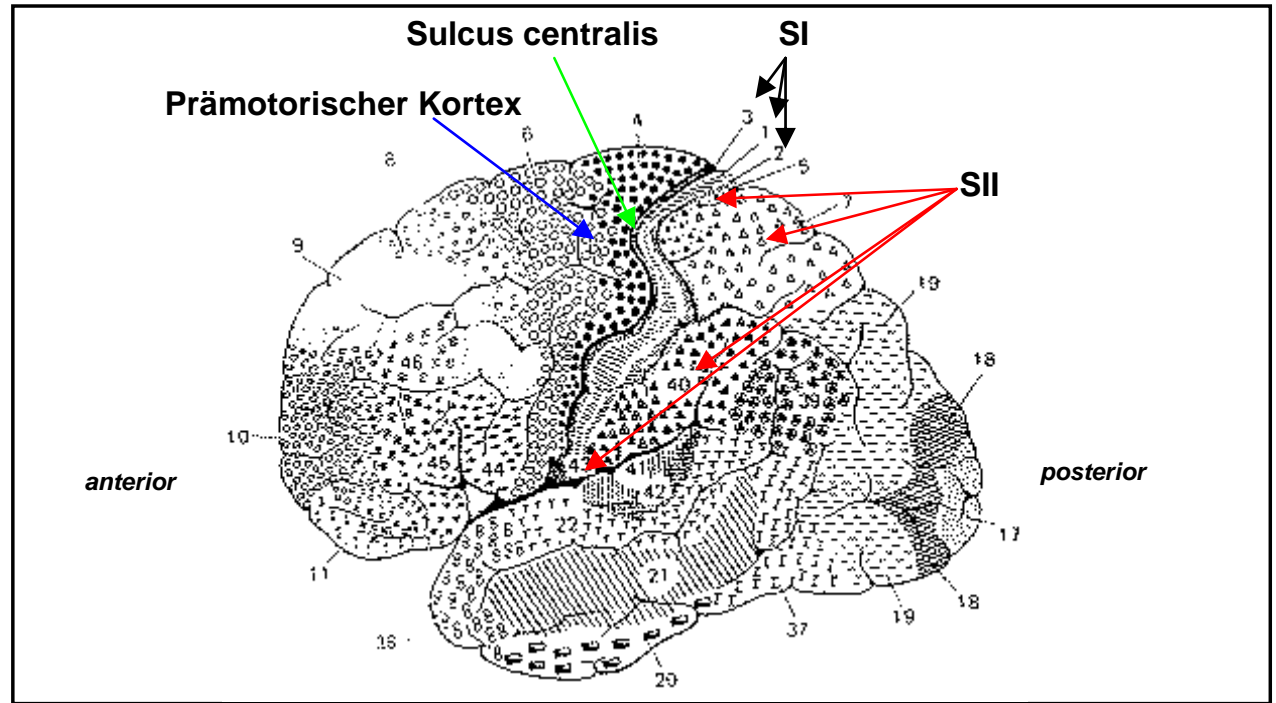

\begin{abstract}
Abbildung 03: Rindenfeldgliederung nach Brodmann. Die Hemisphärenrinde wird nach histologischen Parametern in 52 Rindenfelder eingeteilt. In der vorliegenden Lateralansicht werden diese dargestellt. Die besonders wichtigen Rindenfelder werden in folgenden Texten als solche erwähnt und ihre Funktionen näher erläutert. Die Aufzählung beginnt mit den Brodmann-Arealen 1-3, welche für den Gyrus postcentralis und die SI-Region, stehen. (nach Benninghoff 1994, siehe Trepel 1999, Seite 186).
\end{abstract}

Der N. medianus ist ein kräftiger gemischter, motorischer und sensibler Nerv, der aus den Rückenmarks-Segmenten C5 bis Th1 entspringt. Er entsteht aus dem Fasciculus medialis bzw. lateralis des Plexus brachialis. Anschließend läuft er an der medialen Seite des Oberarms nach distal und passiert ventral die Ellenbeuge. Danach innerviert er sämtliche Unterarmbeuger mit Ausnahme des M. flexor carpi ulnaris und ulnaren Anteil des M. flexor digitorum profundus. Weiterhin innerviert er alle Pronatoren. Vom Unterarm ausgehend innerviert er sensibel die radialen zwei Drittel der Hohlhand. Er passiert den Karpaltunnel. In der Hohlhand innerviert er motorisch die Daumenballenmuskulatur sowie sensibel Teile der Finger I-III. Bei einem Ausfall tritt die sog. Schwurhand auf (Poeck und Hacke 2001). 


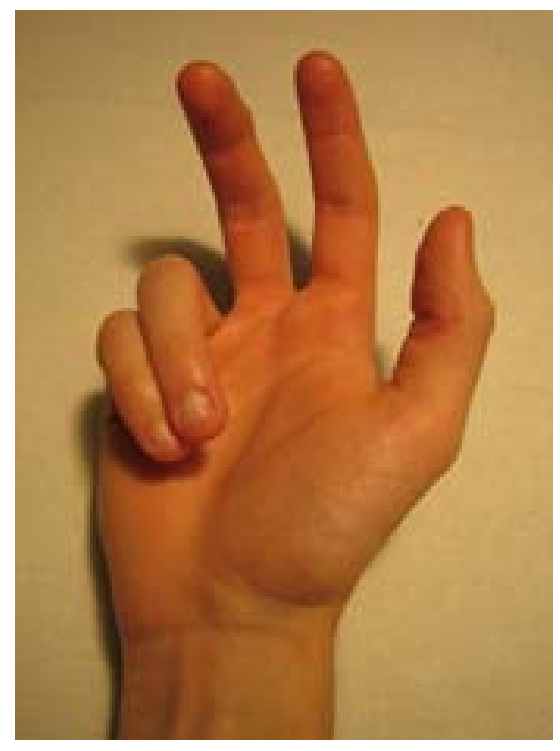

Abbildung 04: Schwurhand bei Läsion des N. medianus. Sensible Innervation des N. medianus: Volarseite der Finger I bis zur radialen Hälfte von IV und angrenzende Hautbezirke der Hand, Dorsalseite der Endglieder I-III. Bei dem Versuch die Finger in den Zwischen- und Endgelenken zu beugen, entsteht die sog. Schwurhand. Nur die vom N. ulnaris motorisch innervierten Finger IV und V und in geringem Maße Finger III können gebeugt werden. Daumen und Zeigefinger bleiben stehen (nach Poeck und Hacke 2001, Seite $625)$.

Das somatosensible System lässt sich folgendermaßen beschreiben. Die sensiblen Informationen lassen sich in Propriozeption und epikritische Empfindungen einteilen. In der Peripherie gibt es Rezeptoren für jede einzelne Qualität, welche die Informationen aufnehmen und an höhere Zentren in afferenten Bahnen senden. Bei den somatosensiblen Wahrnehmungen wird zwischen reiner Empfindung von bestimmten Qualitäten und einer komplexen Kombination von Wahrnehmungen unterschieden. Diese Kombinations-Wahrnehmungen haben keinen eigenen Rezeptor, werden aber dennoch als typisch und einzigartig wahrgenommen. Die Erwartungshaltung bei Empfindungen ist ebenfalls ein wichtiger Faktor, der berücksichtigt werden muss. Drei wichtige Leitungsbahnen sind für die Wahrnehmung eines somatosensiblen Impulses aus der Körperperipherie zuständig, welche die Information an höhere Zentren senden. Dazu zählen die Kleinhirnseitenstrangbahnen (Tractus spinocerebellares anterior et posterior), die Hinterstrangbahnen (Fasciculus cuneatus et gracilis) und die sensiblen Vorderseitenstränge (Tractus spinothalamicis anterior et lateralis) (Trepel 1999). 
Bei der Wahrnehmung der Propriozeption spielen Informationen über die Stellung und Lage der Muskelspindeln und Sehnenorgane eine wichtige Rolle. Diese Informationen werden vom ersten Neuron aufgenommen und sind zuständig für die Muskulatur. Der Informationsfluss verläuft vom Rezeptor über das pseudounipolare Perikaryon im Spinalganglion bis zum zentralen Fortsatz, der über die Radix posterior in das Rückenmark eintritt. Die zentralen Fortsätze steigen anschließend im Hinterhorn des Rückenmarks auf, bis sie den Nucleus dorsalis und Nucleus proprius erreichen. Hier wird die Erregung vom ersten auf das zweite Neuron umgeschaltet. Von diesen beiden Kernen entspringen die Kleinhirnhinterstrangbahnen. Sie werden in einen anterioren und einen posterioren Anteil unterschieden. Der posteriore Tractus spinocerebellaris steigt gleichseitig im Seitenstrang auf, wohingegen der aufsteigende anteriore Tractus spinocerebellaris gleichseitige und gekreuzte Teile besitzt. Alle gekreuzten Leitungsbündel ziehen vor dem Eintritt in das Kleinhirn wieder zurück auf die ipsilaterale Seite, so dass nur Afferenzen aus dem ipsilateralen Rückenmark in die ipsilaterale Hemisphäre des Kleinhirns gelangen. Der Tractus spinothalamicus anterior gelangt dabei über den Pedunculus cerebellaris superior und der Tractus spinothalamicus posterior über den Pedunculus cerebellaris inferior in das Kleinhirn (Trepel 1999).

Unter protopathischer Sensibilität versteht man die Tast-, Temperatur-, Schmerzund grobe Druckempfindung. Neben spezifischen Rezeptoren wie bei der Propriozeption gibt es bei der protopathischen Sensibilität auch freie Nervenenden, die unter anderem Schmerzempfindungen aufnehmen. Der Verlauf der Erregungen entlang des ersten Neurons gleicht der Propriozeption. Über die Radix posterior treten die zentralen Fortsätze der Afferenzen in das Rückenmark ein. Außer den schmerzleitenden Bahnen werden alle restlichen Bahnen im Hinterhorn auf das zweite Neuron umgeschaltet. Die Schmerzafferenzen steigen erst ein bis zwei Rückenmarksegmente auf, um dann in der Substantia gelatinosa auf das zweite Neuron umgeschaltet zu werden. Nach der Verschaltung kreuzen die Leitungsbahnen der protopathischen Sensibilität durch die Commissura alba auf die contralaterale Seite und ziehen als Tractus spinothalamicus im Vorderseitenstrang zum Thalamus (Trepel 1999). 
Die Qualität der Hinterstrangbahnen ist die epikritische Sensibilität. Zur Aufnahme dieser existiert eine große Anzahl von verschiedenen Rezeptoren, die sich in der Zeit ihrer Adaptation und in ihrer Sensibilität auf epikritische Reize unterscheiden. Es wird durch die Rezeptoren nur eine Teilqualität kodiert und zentral weitergegeben. Beispiele für Teilqualitäten sind das Vibrationsempfinden und die Druckveränderung pro Zeit. Zur Übersicht sind hier nur einige Rezeptortypen wie schnell adaptierende Rezeptoren, Vater-Pacini-Körperchen und Golgi-Mazzoni-Körperchen oder langsam adaptierende Ruffini-Körperchen genannt. Aus diesen oder ähnlichen peripheren Rezeptoren wird eine epikritische Information über die Spinalnerven in das Rückenmark zusammen mit den beiden anderen Leitungsbahnen geführt. Sie werden nicht direkt im Hinterhorn umgeschaltet, sondern steigen als Hinterstrangbahnen zum Nucleus cuneatus et gracilis auf. Dort werden sie auf das zweite Neuron umgeschaltet. Die Fasern der zweiten Neurone bilden zusammen den Lemniscus medialis und kreuzen in der Decussatio lemniscorum auf die contralaterale Gegenseite, um dann weiter zum Thalamus zu laufen (Trepel 1999).

Der Thalamus ist die folgende Station, an der die epikritischen und protopathischen Informationen vom zweiten auf das dritte Neuron umgeschaltet werden. Dies trifft nicht für Erregungen zu, die durch den Tractus spinocerebellaris geleitet werden, da dieser im Kleinhirn endet. Der somatosensible Anteil des Thalamus besteht aus den beiden Kernen Nucleus ventralis posteromedialis (VPM) und Nucleus ventralis posterolateralis (VPL). Die Leitungsbahnen aus dem Nervus trigeminus ziehen dabei zum posteromedialen Kern, wohingegen die Fasern des Rückenmarks zum posterolateralen Kern ziehen. Eine Sonderstellung nehmen hier die schmerzleitenden Bahnen ein. Sie sind zusätzlich zu den Kernen des VPL und VPM noch mit anderen unspezifischen Thalamuskernen, wie beispielsweise der anterioren Kerngruppe, verschaltet. Im Gegensatz zu den anderen Wahrnehmungen wird Schmerz wahrscheinlich schon auf Thalamusebene wahrgenommen. Nach der Umschaltung auf das dritte Neuron zieht ein großer Teil seiner Axone durch die Capsula interna zum primären somatosensiblen Kortex in den Gyrus postcentralis. Dabei gilt, dass die afferenten Informationen wegen der Kreuzung der Leitungsbahnen auf die Gegenseite aus der contralateralen Körperseite stammen (Trepel 1999). 
Erst nachdem ein somatosensibles Signal den primären somatosensiblen Kortex (SI) erreicht hat, wird es wahrgenommen. Durch die Verschaltung der afferenten Leitungen der dritten Neurone kommt es zu einer somatotopen Gliederung im SI, bei der Körperteile mit hoher Rezeptordichte überproportional groß repräsentiert sind. In der Abbildung 05 ist der entsprechende Homunkulus dargestellt.

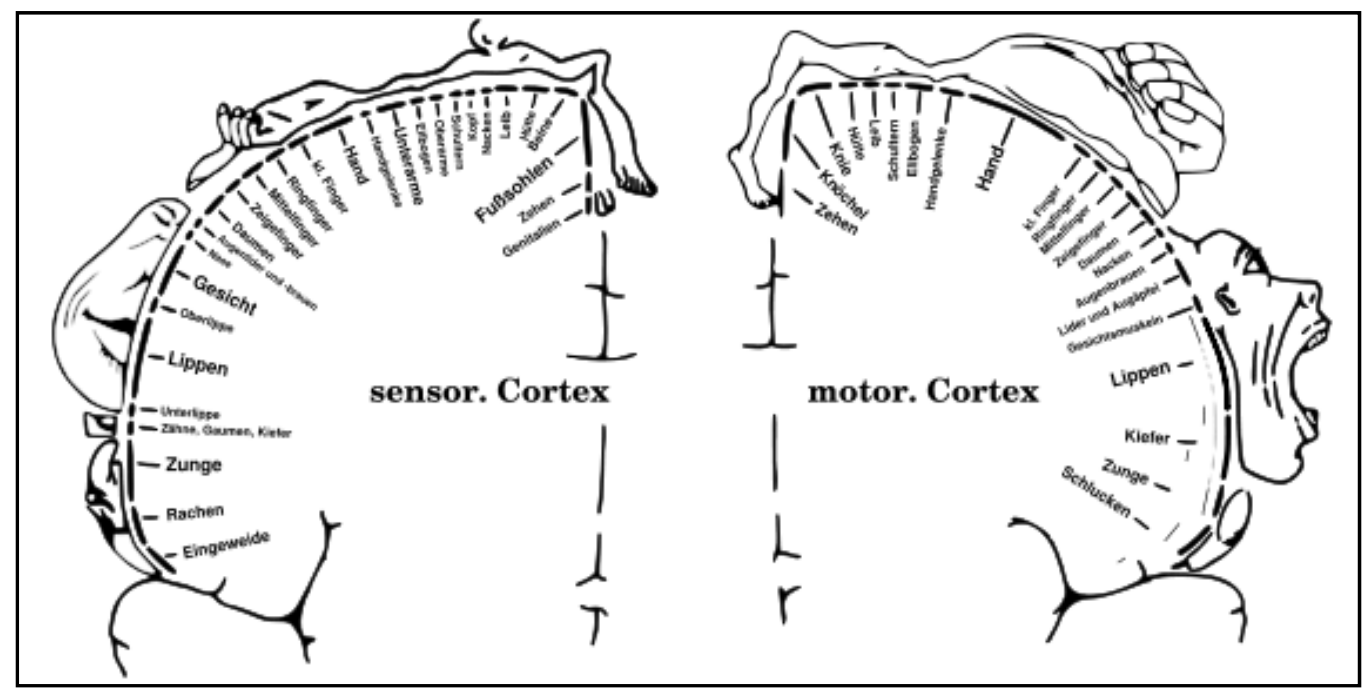

Abbildung 05: Der in der Abbildung dargestellte sensomotorische Homunkulus repräsentiert die Körperareale, die im Kortex mit besonders vielen Sinnesverarbeitungen ausgestattet sind. Dabei nehmen die Hand oder die Lippen beispielsweise relativ große Kortex-Regionen ein, da sie besonders sinnesempfindlich sind. Die Wahrnehmungen vom Rumpf werden dagegen in weniger großen Kortexarealen widergespiegelt. Die Innervation der entsprechenden Kortexareale verläuft contralateral zu der entgegengesetzten Seite zur Sinnes-

Wahrnehmung oder Stimulation (nach Love und Webb 1999, Seite 19).

Der SI-Kortex erstreckt sich von der medialen Mantelkante über den Gyrus postcentralis bis zum Sulcus lateralis. Eingeteilt nach seiner Histologie umfasst der SI nach Brodmann die Areale 1, 2 und 3. Im SI werden die afferenten Impulse vielfach umgeschaltet und weiter in den bilateralen sekundären somatosensiblen Kortex (SII) geleitet. Zu SII gehören nach Brodmann die Areale 5, 7, 40 und 43 sowie insulare Anteile (Abbildung 03). Hier werden die ankommenden Reize, die in SI wahrgenommen wurden, bewertet, interpretiert und mittels Assoziationsfasern mit den restlichen ZNS-Anteilen verknüpft (Trepel 1999).

\subsubsection{Repräsentation des sensorischen Kortex im fMRT}

Es ist derzeit Gegenstand der Forschung und von besonderer klinischer und wissenschaftlicher Bedeutung, wie das somatosensible System in 
Verarbeitungsprozesse mit eingebunden ist und wie es mit der jeweiligen contralateralen Seite kommuniziert. In der Vergangenheit wurden viele Studien mit der Untersuchung von Aktivierungen im Kortex-Bereich, welcher die Finger im sensomotorischen Kortex repräsentiert, durchgeführt. Auch in unserer Studie legten wir einen besonderen Schwerpunkt auf die Analyse dieses Kortex-Bereiches. Die folgende Abbildung 06 hebt in A die unterschiedlichen Bereiche des primären sensorischen Kortex hervor, welche wir bei unseren Messungen besonders betrachteten. S1: Areal 3a, Areal 3b, Areal 1 und Areal 2 (van Westen et al. 2004). In $\mathrm{B}$ wird als Beispiel eine individuelle Aktivierungskarte gezeigt, welche durch die $\mathrm{N}$. medianus-Stimulation zustande kam. Diese zeigt, dass es beispielsweise auf der contralateralen Seite (rot-gelb) zur stimulierten Hand eine positive BOLD-Antwort gab sowie eine negative BOLD-Antwort (blau-grün) in der ipsilateralen Hemisphäre.

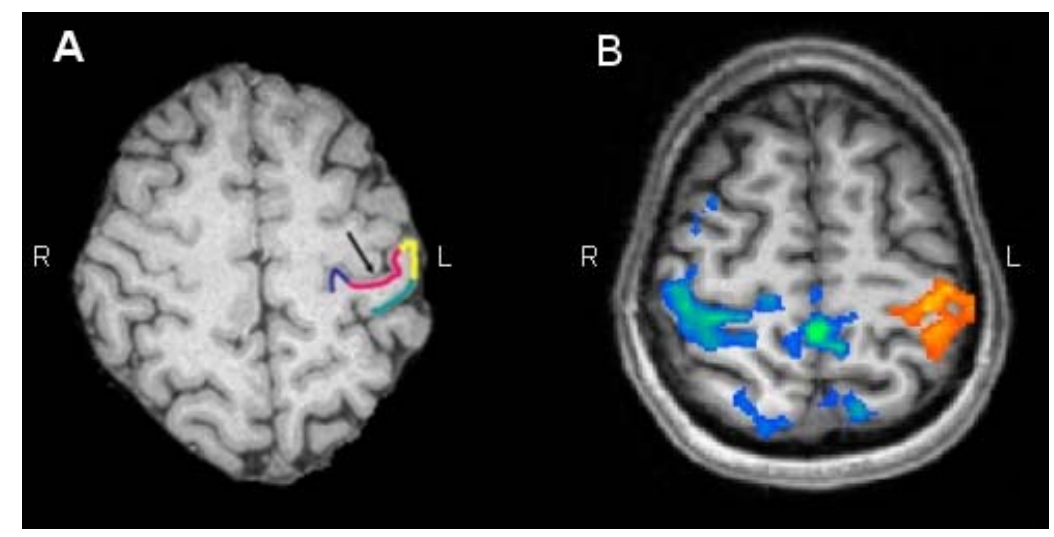

Abbildung 06: fMRT-Repräsentation (A) des primären sensorischen Kortex S1: Areal 3a, Areal 3b, Areal 1 und Areal 2 und Darstellung einer individuellen Aktivierungskarte (B), die durch die N. medianus-Stimulation zustande kam (nach van Westen et al. 2004).

\subsection{Probanden}

An unserer Studie nahmen insgesamt 27 freiwillige Probanden unterschiedlichen Alters teil. Die beiden Gruppen wurden in zwei Altersgruppen eingeteilt, ein älteres und ein jüngeres Probandenkollektiv. In der älteren Gruppe befanden sich 13 ältere, gesunde Probanden ohne zerebrovaskuläre Vorerkrankungen (3 männliche, 10 weibliche, Durchschnittsalter 73,2 \pm 8,3 Jahre) sowie in der jüngeren Gruppe 14 jüngere, gesunde Probanden (6 männliche, 8 weibliche, Durchschnittsalter $23 \pm 1,04$ Jahre). Alle Teilnehmer waren Rechtshänder und unterzogen sich vor den Versuchen medizinischen und neurologischen Untersuchungen zur Erfassung des Allgemeinund Gesundheitszustands sowie möglicher Kontraindikationen für eine fMRTUntersuchung. Dabei wurden eine ausführliche Anamnese und die Aufklärung der 
Probanden durchgeführt. Die Probanden wurden nach besonderen Vorerkrankungen, stattgefundenen Operationen und der Einnahme von Medikamenten befragt. Des Weiteren wurden der Zustand und mögliche Erkrankungen der Atemwege wie Asthma, Allergien und Atemwegsinfekte erfragt. Ebenso befragten wir sie nach Beschwerden des Herzkreislaufsystems wie beispielsweise Herzschwäche, Herzrhythmusstörungen, Herzinfarkt und Synkopen oder Erkrankungen des Bewegungsapparates wie beispielsweise Lähmungen, körperliche Schwäche, allgemeine neurologische Ausfälle und Arthritis. Die Probanden wurden hinsichtlich der relativ engen räumlichen Verhältnisse während der fMRT-Messungen nach möglicherweise bestehender Platzangst befragt. Ebenso ob eine Schwangerschaft vorliegt. Da bei unseren Messungen keine Kontrastmittelgabe (KM) notwendig war, konnten mögliche Komplikationen, die mit KM hätten eintreten können, ausgeschlossen werden. Im Focus unseres Interesses standen neben den oben genannten Fragen vor allem die möglichen Interaktionen von implantierten oder am Körper getragenen Metallteilen wie Prothesen, Stents, Herzschrittmacher, Schmuck oder Zahnimplantaten. Diese sollten wenn möglich vor der Messung abgelegt werden, falls sie nicht zu fest installiert waren. Zusammenfassend stand in erster Linie die Sicherheit der Probanden während der Messungen im Vordergrund. Sie sollten absolut keiner Gefahr oder Nachteilen nach oder während der Messung ausgesetzt werden. Die Probanden stellten sich aus dem Bekannten- und Nachbarschaftskreis der Doktoranden zusammen und waren keine Patienten, die akut auf einer medizinischen oder neurologischen Station behandelt wurden. Sie waren gesundheitlich nicht eingeschränkt und führten alle ein selbstständiges soziales und zum Teil berufliches Leben. Unter den älteren Probanden waren keine abhängigen Pflegefälle oder multimorbiden Probanden. Die Aufklärung erfolgte neben den mündlichen Befragungen und Gesprächen mit den Doktoranden durch das Personal der fMRT-Bedienung gemäß dem Frage- und Aufklärungsbogen der Abteilung MR-Forschung in der Neurologie und Psychiatrie im Bereich Humanmedizin. Dabei ging es neben den Hinweisen auf Sicherheitsaspekte um mögliche Gefahren und deren Vermeidung. Die Probanden wurden vorab darauf hingewiesen, 6 Stunden vor der fMRT-Untersuchung kein Koffein und 24 Stunden vorher keinen Alkohol zu sich zu nehmen. Weiterhin wurden die Probanden über die Pseudonymisierung der akquirierten Datensätze aufgeklärt. Damit ist das Ersetzen ihres Namens durch einen Code gemeint (zum Beispiel: „hum_1628“ für Probanden- 
Name). Ihre Daten sollten ausschließlich von den betreuenden Ärzten, Doktoranden und Mitarbeitern der Arbeitsgruppe bearbeitet und analysiert werden. Alle Probanden gaben vorher ihre schriftliche und formale Einwilligung. Die Studie wurde von der lokalen Ethikkommission bewilligt und die Durchführung der Messungen war in Übereinstimmung mit der Vereinbarung von Helsinki (Weltärztebund 2004) und damit aus ethischer, rechtlicher und sozialer Sicht sowie zum Schutz der Probanden vor den Folgen unserer Messungen unbedenklich und durchführbar (MR-Forschung Göttingen 2006).

\subsection{Methode}

\subsubsection{Sensorische Stimulation mit $40 \mathrm{~Hz}$}

Für die elektrische Stimulation mit $40 \mathrm{~Hz}$ benutzten wir einzelphasischen Konstantstrom mit Bit-Impulsfolge und einer Laufzeit-Dauer von $200 \mu \mathrm{s}$. Das bedeutete, dass kleine Stromstoß-Impulse mit einer hohen Frequenz kurzzeitig hintereinander abgegeben wurden. Bei $40 \mathrm{~Hz}$ wurden diese vom Probanden als eine kontinuierliche Strom-Stimulation wahrgenommen. Ausgelöst wurden die Impulse von dem im neurophysiologischen Klinik-Alltag bewährten Konstant-StromstärkenStimulator (Stimulator Type 3072, Devices Sales LTD, Welwyn Garden City, Hertfordshire, UK) zur direkten Stimulation des rechten N. medianus am Handgelenk. Aus der Erkenntnis von vorherigen Studien-Ergebnissen wurde für diese Studie eine Stimulationsfrequenz von $40 \mathrm{~Hz}$ ausgesucht (Kampe et al. 2000). Die Stromstärke wurde vorher außerhalb des Scanner-Raums individuell ausgesucht sowie an die Motor-Reizschwelle angepasst. Diese ist definiert als die geringste Stromstärke, die eine sichtbare Kontraktion des Daumens auslöste. 


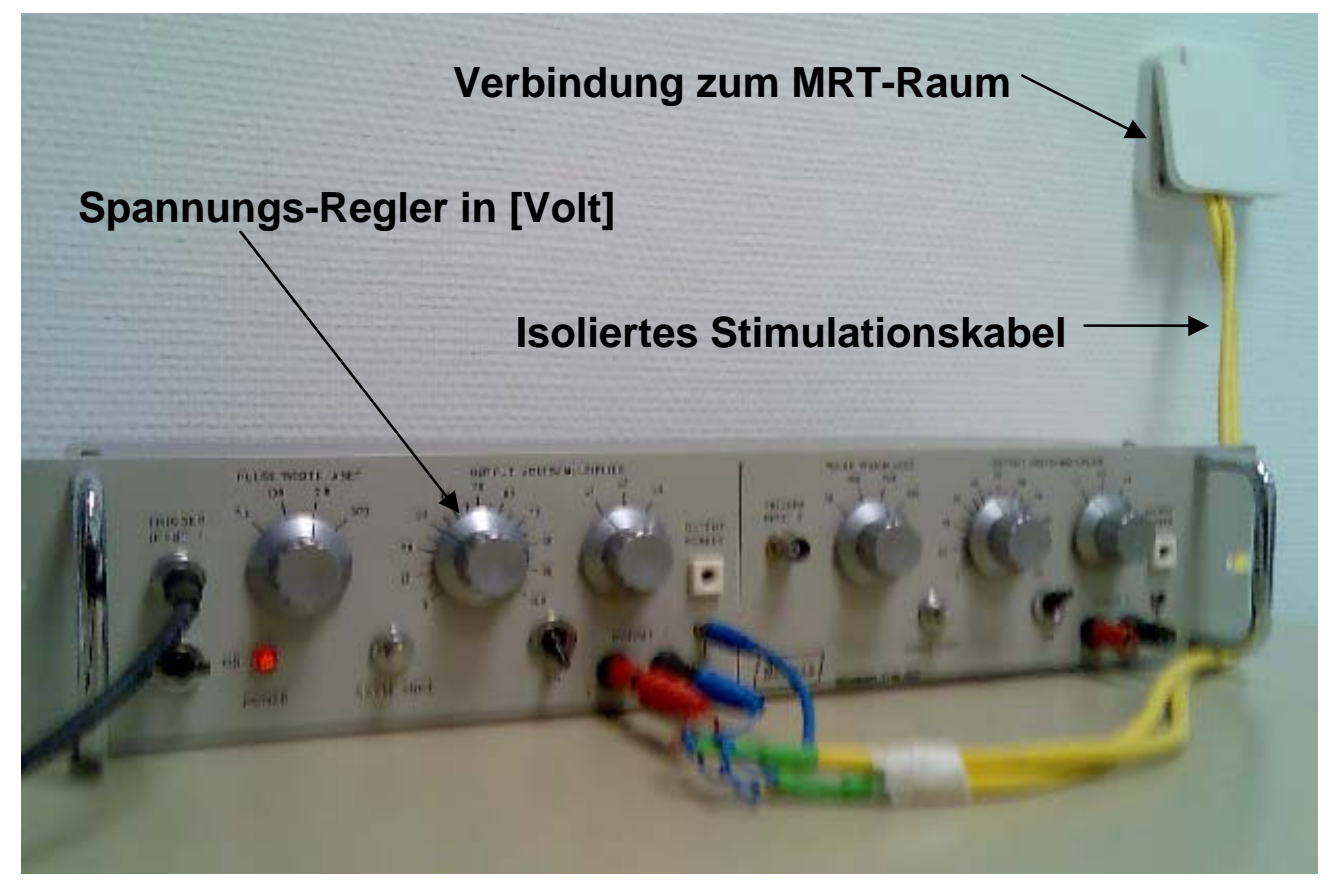

Abbildung 07: Klinischer Stimulator für die Erregung des N. medianus. Stimulator Type 3072, Devices Sales LTD, Welwyn Garden City, Hertfordshire, UK.

Nach anschließender Lagerung und Einschleusen des Probanden in die MRT-Röhre wurde die korrekte Lage der Stimulationselektrode und eine sichtbare Kontraktion der Thenar-Muskulatur noch einmal überprüft und bei etwaigen Verschiebungen der Lage diese wieder angepasst. Unter Berücksichtigung der unterschiedlichen Schmerzschwelle des jeweiligen Probanden war es ein individuelles Ausjustieren der idealen Lokalisation der N. medianus-Stimulation und Balance zwischen minimaler sichtbarer Kontraktion und Berücksichtigung der Schmerzschwelle. Die isolierten Kabel liefen von der Stimulationselektrode durch ein Loch in der Wand des ScannerRaums in einen angrenzenden Raum, wo sich der Stimulator befand und ein Versuchs-Mitarbeiter diesen ständig auf seine korrekte Funktion und Tätigkeit überwachte. Zur Unterstützung der Leitfähigkeit des Stromflusses wurden die Stimulationselektroden mit einem Gel (Medical Systems Information Technologies $\mathrm{GmbH}$ ) benetzt. Anschließend wurde diese auf der Haut auf Höhe der anatomischen Lage des rechten $\mathrm{N}$. medianus mit hautfreundlichem Klebepflaster fixiert. 


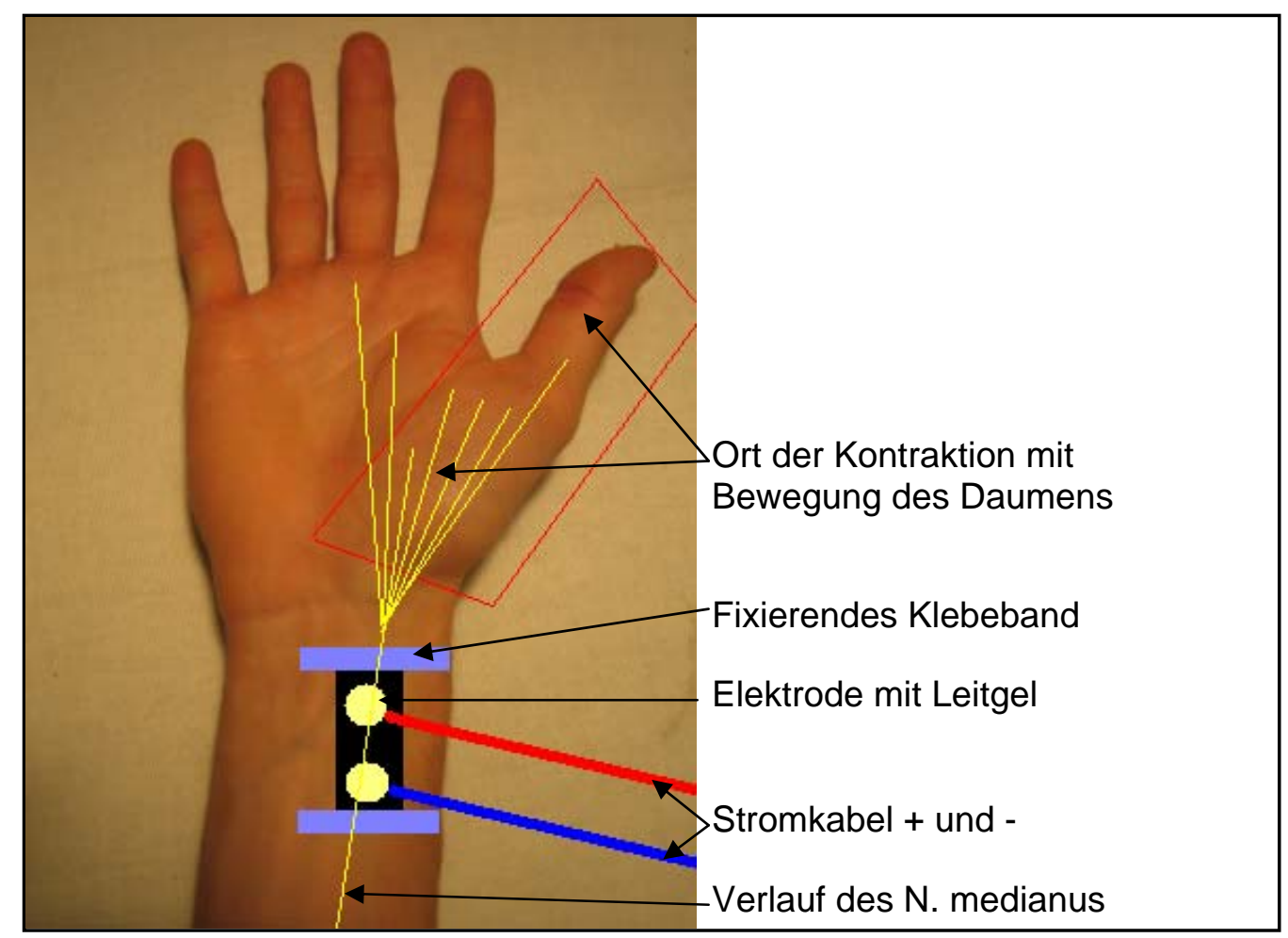

Abbildung 08: Darstellung der Fixierung der Stimulationselektrode in der Region des rechten Handgelenks. Zusätzlich als wichtiger Ort von Interesse der Bereich, wo eine mögliche Kontraktion oder Daumenbewegung sichtbar gewesen sein sollte.

Als ein wichtiges Kriterium erfuhr kein Proband das Gefühl von Schmerz (Nihashi et al. 2005). Dieser hätte sonst die Aktivierungskarten in der anschließenden Auswertung der fMRT-Daten stark beeinflusst und fokussierte Kortexareale mit Schmerzsignalen überdeckt und somit nicht ausreichend differenziert dargestellt. Die elektrischen Stimulations-Apparate verursachten keine Artefakte in den fMRTBildern. Das Stimulationsprotokoll begann mit einer Nicht-Stimulations-Phase (die Ruhephase von 30 Sekunden). Daran schloss sich eine 30 Sekunden andauernde 40-Hz-Stimulation an, gefolgt von einer erneuten Ruhephase, die ebenfalls 30 Sekunden andauerte. Dieses wurde $6 \mathrm{Mal}$ wiederholt und ergab einen GesamtStimulations-Block von 6,5 Minuten (40-Hz-Runde 1). Eine zweite funktionelle Messung (40-Hz-Runde 2) wurde mit denselben Parametern eine Minute später gestartet. Vor Beginn der Stimulationsdurchgänge wurde bei jeder Messung ein anatomischer Datensatz erstellt. 


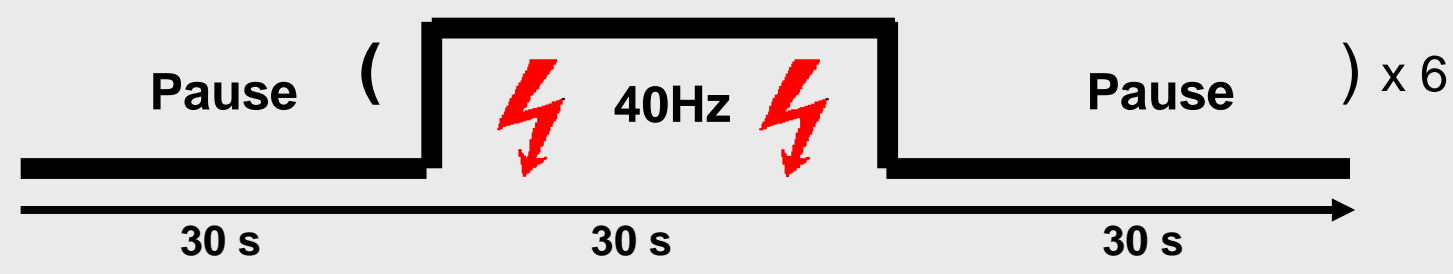

Abbildung 09: Stimulationsdurchgang als Block-Design der 40-Hz-Messungen.

Bei der gesamten Messung und auch den folgenden war es wichtig, dass sich die Probanden nicht bewegten damit korrekte Aufnahmen und Messungen entstehen konnten. Sie sollten die Messungen passiv über sich im liegenden, nicht bewegtem Zustand ergehen lassen und nur auf Anweisung antworten. Eine Ausnahme war es, wenn sie sich in einer unwohlen Notsituation befanden oder Schmerzen jeglicher Art verspürten. Wichtig war es zusätzlich, dass die Probanden nicht einschliefen. Studien zeigten, dass bei schlafenden Probanden keine BOLD-Signal-Änderungen im fMRT zu sehen sind (Kampe et al. 2000).

1.

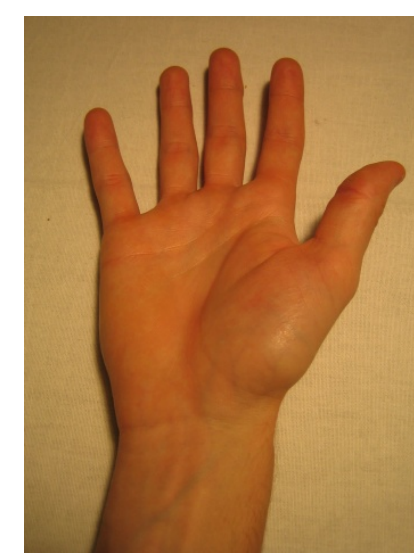

Keine

Stimulation
2.

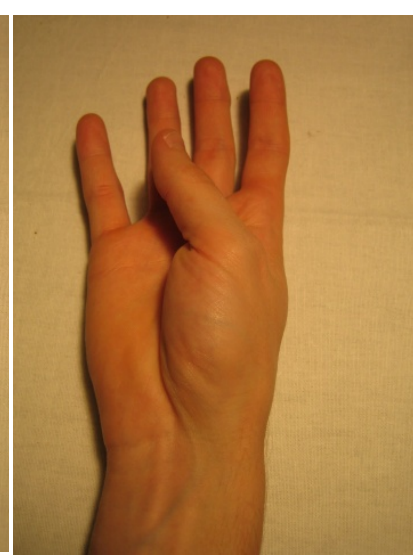

Leichte

Stimulation
3.

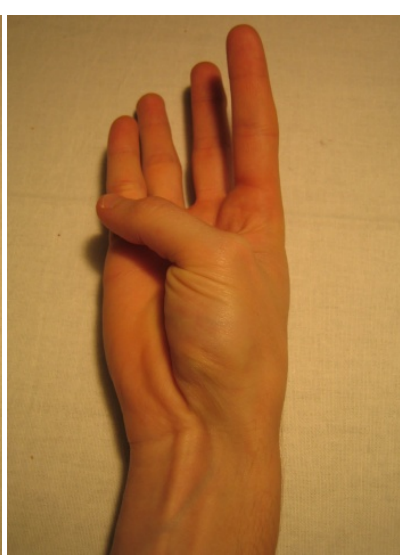

Starke Stimulation
4.

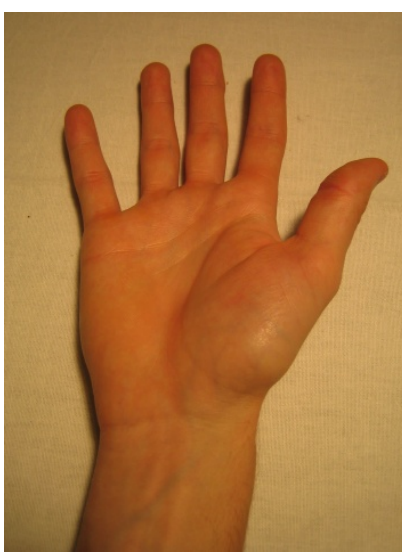

Keine

Stimulation

Abbildung 10: In dieser Fotoserie wird der Ablauf einer Stimulation mit $40 \mathrm{~Hz}$ dargestellt. Man erkennt, dass durch Stimulation des rechten N. medianus der Daumenballen und dessen versorgende Muskulatur zur Kontraktion gebracht werden. Der Daumen bewegt sich nach links. Je stärker die Stimulation, desto stärker ist die Kontraktion der Muskulatur, wie man in Bild 3 erkennen kann. 


\subsubsection{Finger-Tapping (FT)}

Nach 2 Durchgängen der Stimulation mit $40 \mathrm{~Hz}$ führten wir ein so genanntes FingerTapping durch, bei dem aktiv die Finger der rechten Hand im Wechsel mit dem Daumen in Kontakt traten. Dieses wurde unter visueller Vorgabe durchgeführt. Bei einem grünen Punkt im Display der aufgesetzten Präsentations-Brille im MRT mit integriertem Bildschirm sollten die Probanden die Aktion durchführen. Bei einem roten Punkt pausieren und die Hand ruhen lassen. Wichtig war es hierbei, dass die Probanden das Gegenüberstellen der Finger und des Daumens auch an der rechten Hand, wo mit $40 \mathrm{~Hz}$ stimuliert wurde, durchführten. Das FT wurde bei allen Probanden als Abschlussmessung im MRT in einem Gesamt-Zeit-Block von 4,3 Minuten durchgeführt.

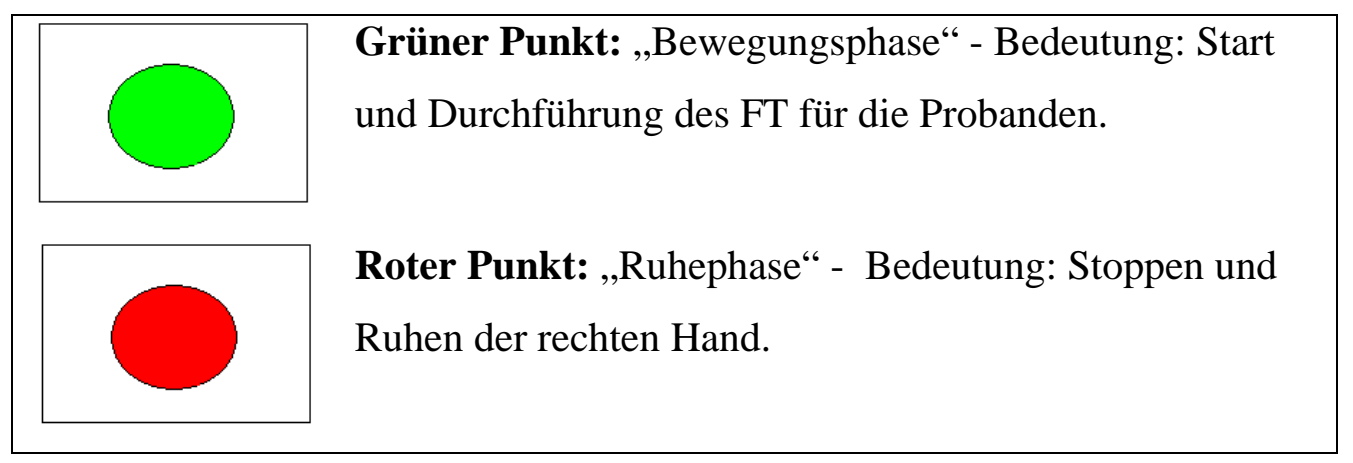

Abbildung 11: Darstellung der Probanden-Anweisungen.

1.

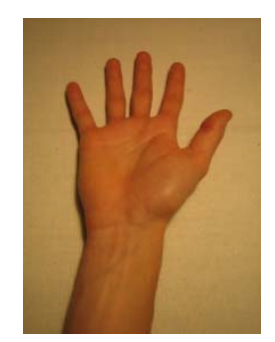

Ruhephase

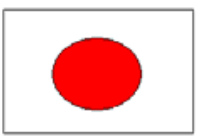

2.

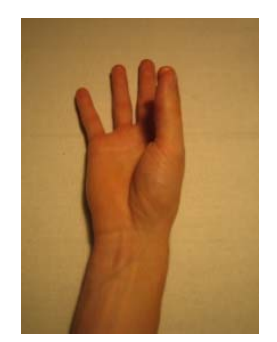

Daumen vs. Zeigefinger

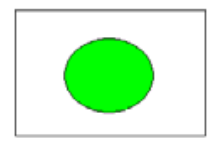

3.

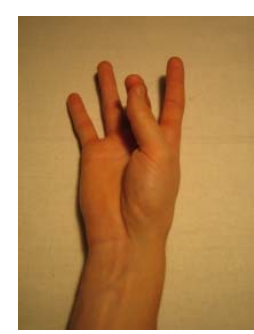

Daumen vs. Mittelfinger

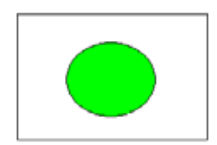

4.

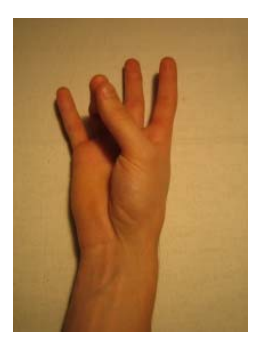

Daumen vs.
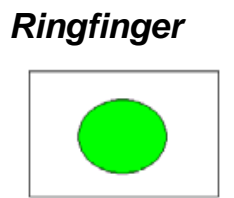

5.

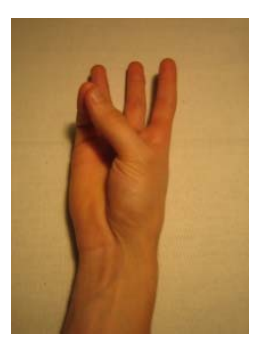

Daumen vs.

Kleiner Fing.

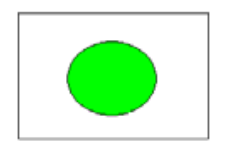

6.

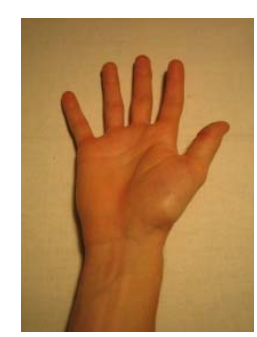

Ruhephase

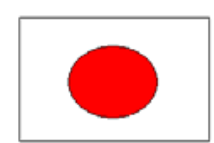

Abbildung 12: In der Fotoserie wird der Ablauf eines typischen von uns durchgeführten Durchgangs des FT dargestellt. Im Verlauf sieht man die einzelnen Bilder mit der Erklärung der jeweiligen Tätigkeit. Die roten und grünen Zeichen verdeutlichen: Aktion = grün und Ruhephase $=$ rot. 


\subsection{3 fMRT-Daten-Erfassung}

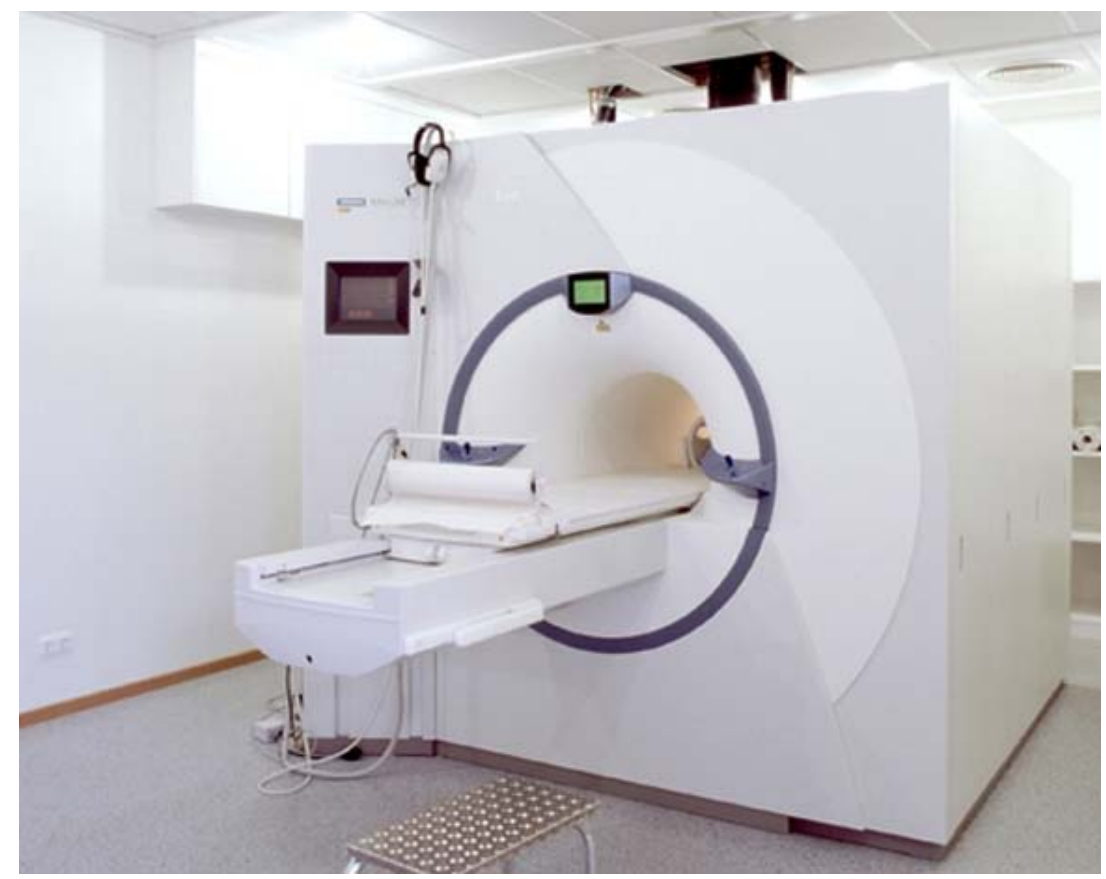

Abbildung 13: 3 Tesla MRT, Siemens Magnetom Trio, Erlangen, Deutschland. MR Forschung in der Neurologie und Psychiatrie, im Bereich Humanmedizin, im Universitätsklinikum der Georg-August-Universität Göttingen.

Die fMRT-Aufnahmen wurden mit einem 3 Tesla MRT durchgeführt (Siemens Magnetom Trio, Erlangen, Deutschland, siehe Abbildung 13). Dabei wurde eine Hauptspule mit einem Standard-Acht-Kanal und einem in Phasen angeordnetem System benutzt. Die Probanden wurden in Rückenlage in die Magneten-Röhre gelegt und trugen Kopfhörer für den Lärmschutz sowie Polster zur bequemeren Lagerung unter den Knien und Polster für den Schutz des Kopfes unter der MRT-Maske mit Bildschirm-Brille. Bei Bedarf wurde innen eine Decke gegeben. Mit dem Kopf voraus wurden sie anschließend unter Kontrolle des MRT-Bedienungs-Personals automatisch in die Magneten-Röhre gefahren. Die Vitalparameter wie beispielsweise Puls und die periphere arterielle Oxygenierung wurden während des gesamten Experiments auf einem außerhalb des Scanners sichtbaren Monitor dargestellt. Ein "Not-Knopf" wurde den Probanden in die linke Hand gelegt. Die MRT-Maske mit integrierter Brille mit Bildschirm diente zum Angeben von Anweisungen beim späteren FT. Sonst war der Bildschirm bei den Messungen ausgeschaltet um keine optischen Einflüsse auszuüben. Die Rohdaten der Messungen bestehen aus zwei Anteilen, dem funktionellen und dem anatomischen Anteil. Der anatomische Anteil 
besteht im Einzelnen aus dem zuerst erstellten hochaufgelösten T1-gewichteten 3DMR-Datensatz, mit welchem der gesamte Kopf in sagittaler Orientierung mit einer Auflösung und Voxelgröße von $1 \times 1 \times 1 \mathrm{~mm}$ isotropische Voxel dargestellt wurde (Dreidimensionale Aufnahme - 3D Turbo Fast Low Angle Shot - Sequenz 3DFLASH). Es wurde eine Wiederholungs-Zeit (TR: Iime of Repetition) von $1950 \mathrm{~ms}$ verwendet. Die Umkehr-Zeit (TI: Time of Inversion) lag bei $1100 \mathrm{~ms}$. Die Echo-Zeit (TE: Iime of Écho) bei $3,93 \mathrm{~ms}$. Der Flip-Winkel (Flip Angle) wurde auf $12^{\circ}$ eingestellt. Ein Voxel ist das Volumen-Element, welches in Beziehung steht zu einem Pixel. Ein Pixel ist wiederum das einzelne Element oder eine zweidimensionale Anordnung von Bilddaten. Das Voxel-Volumen ist gleich zum Pixel-Areal, vervielfacht durch die Schicht-Dicken. Dieser Datensatz diente zur Aufnahme der normalen anatomischen Verhältnisse und Strukturen des Kopfes und damit des ZNS. Für die funktionellen Aufnahmen wurde eine T2-sensitive Gradienten-Echo-EPI-Technik mit einer auf gleicher Ebene liegenden Auflösung von $2 \times 2 \mathrm{~mm}^{2}$ benutzt. Die TR lag bei $2000 \mathrm{~ms}$, die TE bei $36 \mathrm{~ms}$, der Flip Angle bei $70^{\circ}$ und die Akquisitions-Matrix bei 128x96. Unter der Echo-EPI-Technik versteht man eine Pulssequenz, die benutzt wird, um sehr schnelle MRT-Aufnahmen zu produzieren. Diese können bis unter 50 ms kurz sein. Es wurden 22 aufeinander folgende Schichten-Abschnitte von $4 \mathrm{~mm}$ Dicke erstellt und in einer axial zu koronal Ausrichtung gewinkelt. Aus den oben genannten Aufnahmen setzen sich die Rohdaten, die für die Weiterverarbeitung benötigt werden, zusammen. Mit dem funktionellen Datensatz wurden Aktivierungskarten erstellt, um später in der Auswertung die Areale zu sehen, in denen Aktivität stattfand, und Areale, in denen eventuell deaktiviert wurde (positive und negative BOLD-Signal-Änderungen). Für jeden der zwei experimentellen Durchläufe wurden insgesamt 195 Bildsätze aufgezeichnet.

\subsection{4 fMRT-Daten-Analyse}

Die Daten-Analyse wurde mit der Software BrainVoyager QX 1.7 (Brain Innovation Maastricht, The Netherlands) durchgeführt. Die Analyse der fMRT-Daten wird in mehrere Arbeitsschritte unterteilt. Im ersten Schritt, dem Preprocessing (Vorverarbeitung) der Datensätze, werden die Daten für die statistische Auswertung vorbereitet. Sie werden von Bewegungsartefakten und Signalsprüngen befreit und anschließend in den stereotaktischen Raum nach Talairach übertragen. Dem 
Preprocessing folgt die Definition des statistischen Modells und die statistische Analyse der Daten mit Erstellung des statistischen Modells, statistische Auswertung und die graphische Darstellung der Ergebnisse. Für die korrekte graphische Darstellung der Ergebnisse muss man neben den eigentlichen fMRT-Daten zusätzlich einen hochaufgelösten 3D-Datensatz aufnehmen (Goebel und Jansma 2004). Das Vorgehen beinhaltete Hilfsmittel zum Preprocessing, zur Registrierung, statistischen Auswertung und der Präsentation von fMRT-Daten. Die Vorverarbeitung umfasst 3D Motion Correction, Slice Scan Time Correction, Linear Trend Removal und Spatial Smoothing mit Gauß'scher Verteilung (Gaussian Kernel, mit einer vollen Bandbreite mit halben Maximum bei $8 \times 8 \times 8 \mathrm{~mm}^{3}$ ). Nach manueller Auffindung der vorderen und hinteren Kommissur wurden die anatomischen Datensätze entlang des Talairach-Koordinatensystems ausgerichtet. Dabei verläuft der Nullpunkt durch die vordere Kommissur, die x-Achse in sagittaler Richtung durch die hintere Kommissur und die $y$ - und z-Achse entsprechend im $90^{\circ}$ Winkel dazu.
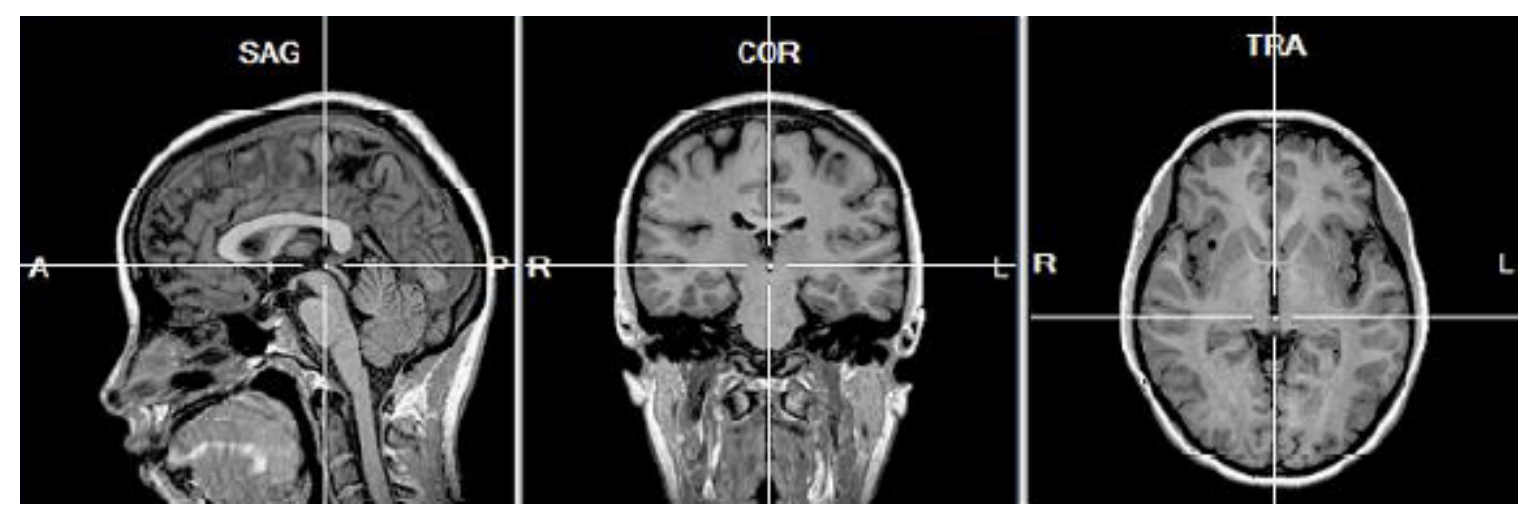

Abbildung 14: Ausrichtung des anatomischen Datensatzes am Talairach-Koordinatensystem (Talairach und Tournoux 1988).

Anschließend wurden die funktionellen Daten auf den korrespondierenden anatomischen Datensatz coregistriert. Es gibt zwei Formen des Coregistrierens. Zum Einen wird im Rahmen der Bewegungskorrektur coregistriert, denn jede fMRTMessung hat bis zu 120 oder mehr Einzelbilder. Daraus wir ein Gesamtbild errechnet. Durch Bewegungsartefakte sind Einzelbilder aber nicht immer Deckungsgleich. Durch die Coregistrierung wird jedes Einzelbild im Raum genau in seiner Position definiert. Dadurch werden die Verschiebungen der Einzelbilder errechnet. Anhand dieser Daten wird die Bewegungskorrektur durchgeführt. Die zweite Form des Coregistrierens dient der Fusionierung der Bilder unterschiedlicher Modalitäten wie fMRT-EPI-Bilder und 3D-MPR-Bilder. Anschließend wurden die 
funktionellen Daten mit dem General Linear Model (GLM) analysiert. Das Modell wurde erstellt mit Hilfe der Windungen der Boxcar-Funktion vom Zeitverlauf (Timecourse) der elektrischen Stimulationen mit einer standardisierten, hämodynamischen Antwort-Funktion (two-gamma HRF). Für die Einzel-ProbandenAnalyse wurden beide Durchgänge in die Analyse der BOLD-Antworten des Stimulations-Paradigmas mit einbezogen und ergaben individuelle Aktivitäts-Karten (Activation Maps). Standardmäßig gilt bei BrainVoyager für die Aktivitäts-Mappen die Fehlerwahrscheinlichkeit (FDR) für statistische Schwellenwerte. Diese Herangehensweise bezieht eine Korrektur für multiple Vergleiche und Kontrollen für falsch-positive Voxel mit ein, als eine Teilmenge von Voxeln, die als signifikant klassifiziert sind. Die FDR wurde bei $q<0,05$ gesetzt mit der Erwartung, dass $5 \%$ der Voxel als falsch-positiv klassifiziert werden. Um in der Lage zu sein, individuelle Veränderungen von BOLD-Antworten für die Korrelation mit psychophysiologischen Daten zu erhalten, wurde eine Region-of-Interest (ROI) im beispielsweise ipsilateralen Finger-Areal (siehe: 2.1.2 Repräsentation des sensorischen Kortex im fMRT) auf dem 3D anatomischen Datensatz platziert sowie später in der umfassenden Auswertung auch auf anderen Kortexarealen. Weiterhin wurde die prozentuale Veränderung des BOLD-Signals von der individuellen Zeit-Lauf-Analyse des coregistrierten funktionellen Datensatzes aufgenommen. Für die statistische Gruppenanalyse wurde eine Random-Effekt-Analyse angewendet. Ebenfalls wurde die FDR bei einem Level von 0,05\% benutzt, um einen Schwellenwert für GruppenAktivitäts-Karten zu setzen. Abschließend wurde der funktionelle Zeitverlauf berechnet, um die Veränderungen des BOLD-Signals über die Zeit innerhalb der Gruppe zu beschreiben (Goebel und Jansma 2004).

\subsubsection{Psychophysiologische Messungen (CPT)}

Vor den Messungen wurde den Probanden der Ablauf der Messung ausführlich beschrieben und mögliche ungeklärte Fragen beantwortet. Zum Ausmessen der quantitativen sensorischen Funktion wurde durch elektrische Stimulation des linken Zeigefingers dessen sensibler Reizschwellenwert (CPT: Cuurrent Perception Threshold) getestet, während gleichzeitig eine hochfrequente, elektrische Stimulation des rechten N. medianus erfolgte. Ebenso maßen wir die CPT in Ruhephasen vor und nach der Stimulation. Dabei wurde die gleiche Stimulations-Apparatur und der 
gleiche Protokoll-Ablauf wie in den fMRT-Messungen mit der 40-Hz-Stimulation zuvor benutzt (siehe: 2.3.1 Sensorische Stimulation mit $40 \mathrm{~Hz}$ ). Wie schon bei den Messungen im MRT wurden für den jeweiligen Probanden dieselben Spannungswerte wie für die $40-\mathrm{Hz}-$ Stimulation eingestellt. Die CPT ist die minimale Menge von transkutan angewandter Stromstärke, welche bei einem Individuum eine reproduzierbare Sinnesempfindung hervorruft. Kürzlich wurde dieses Verfahren als eine einheitliche Methode zum Ermitteln sensorischer Funktion vorgeschlagen (Blankenburg et al. 2003, Davey et al. 2001, Yamashita et al. 2002). Für die Experimente waren die Probanden hinsichtlich der Ergebnisse des FMRT blind ausgewählt worden. Aus organisatorischen Gründen führten wir die psychophysiologischen CPT-Messungen bei jeweils 9 Probanden der zwei Altersgruppen durch. Um für die gleichen Bedingungen während der psychophysiologischen und den zuvor erfolgten fMRT-Messungen zu sorgen, wurde die CPT-Messung während einer laufenden MRT-Messung in dem Raum benachbart zum MR-Scanner abgewickelt, um so insbesondere die Geräusche der ScannerProzedur als ablenkende Fehlerquelle zu berücksichtigen. Die Probanden wurden angewiesen, sich, so gut sie können, zu entspannen, um zu gewährleisten, dass die Grundmuskulatur die Haut nicht auf ein höheres Niveau vorspannte. Die Probanden sollten die Augen schließen und sich auf ihren linken Zeigefinger konzentrieren. Dabei sollten sie darauf achten nicht einzuschlafen, da dies ebenfalls das Ergebnis verfälschen würde.

\subsubsection{Simultane Messungen}

In einzelnen Versuchen nahmen wir später 4 Probanden heraus und versuchten die psychophysiologsichen Messungen als Einzelbeispiele auch im Scanner selbst durchzuführen. Die Ergebnisse im und außerhalb des Scanners waren dieselben. Aus organisatorischen Gründen bevorzugten wir die psychophysiologischen Messungen hauptsächlich im benachbarten Raum durchzuführen, damit für alle Probanden die gleichen Versuchsbedingungen gewährleistet wurden. 


\subsubsection{CPT-Testlauf}

Die Probanden saßen am Tisch mit den Unterarmen aufgelegt. Am rechten Unterarm wurde innen an dieselbe Stelle wie schon im MRT die Zwei-Punkt-Elektrode angebracht. Anfänglich wurde ein erster Testvorlauf durchgeführt, bei dem die Stimulations-Stromstärke angepasst wurde, bei einer Intensität, welche klar am linken Zeigefinger vom Probanden wahrgenommen wurde. So konnte dem Probanden bewusst werden, wie sich die Stimulation anfühlen würde, um sie klar von anderen Einflüssen, wie beispielsweise dem eigenen Puls, zu unterscheiden. Dies erfolgte durch eine Elektrode, welche mit dem Stimulator, dem Digitimer D185-EH (Digitimer LTD, Welwyn Garden City, Hertfordshire, UK), verbunden war. Einstellungen am Digitimer sahen folgendermaßen aus. Die Spannung war variabel eingestellt, bei 0 beginnend und bis zur subjektiven Reizschwelle zunehmend. Die Elektroden wurden in solch einer Position gehalten, dass sie auf der palmaren Seite auf Höhe des Mittleren- und End-Fingerglieds des linken Zeigefingers fixiert wurden. Zusätzlich wurde die Elektrode durch ein Plastikband gesichert und zur Erhöhung der Leitfähigkeit mit einem speziellen medizinischen Leitgel (Medical Systems Information Technologies $\mathrm{GmbH}$ ) benetzt, um den Hautwiderstand zu verringern. Nach dem Testlauf wurde die Spannung wieder auf Null gestellt.

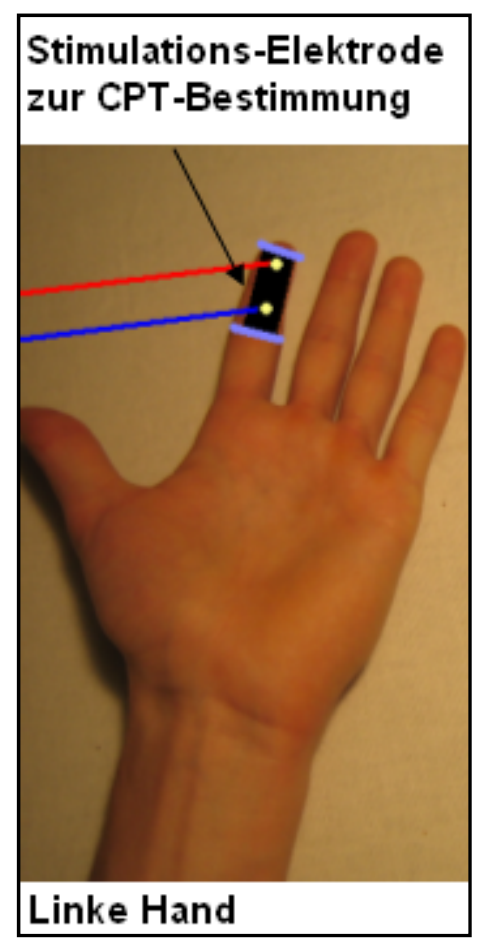

Abbildung 15: Dargestellt ist die Anbringung der Elektrode zur CPT-Messung am linken Zeigefinger. 


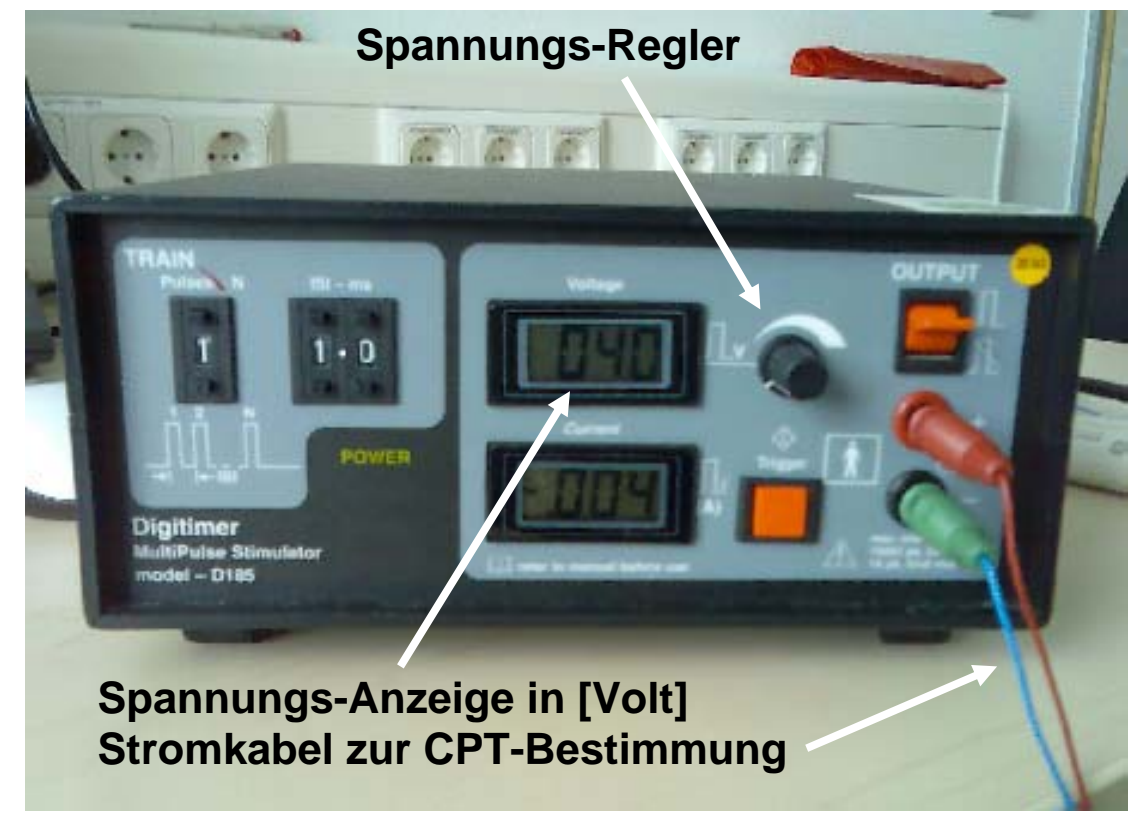

Abbildung 16: Stimulator zur Bestimmung der CPT am linken Zeigefinger. Digitimer D185EH, Digitimer LTD, Welwyn Garden City, Hertfordshire, UK.

\subsubsection{CPT-Messungen unter 40-Hz-Stimulation}

Um in der Lage zu sein die Ergebnisse der fMRT-Messungen mit den psychophysiologischen Experimenten zu vergleichen, wurden bei diesem Experiment die gleichen Protokoll-Einstellungen ausgewählt wie bei den fMRT-Messungen mit der 40-Hz-Stimulation zuvor (siehe: 2.3.1 Sensorische Stimulation mit $40 \mathrm{~Hz}$ ). Es wurde wieder eine 30 Sekunden andauernde $40-\mathrm{Hz}-$ Stimulation am rechten $\mathrm{N}$. medianus angewendet mit einer Stimulations-Elektrode (Stimulator Type 3072, Devices Sales LTD, Welwyn Garden City, Hertfordshire, UK), die in etwa 1-3 cm proximal des Handgelenks angebracht wurde. Dabei wurde die anatomische Lage so gewählt, um möglichst den in der Tiefe verlaufenden N. medianus ideal zu stimulieren. Die Einstellungen des Stimulators für die rechte Hand sahen folgendermaßen aus. Die Impulsdauer betrug 200 ms und die Spannung war variabel, unterhalb der Schmerzschwelle des jeweiligen Probanden. Diesem Durchgang folgte ebenfalls eine 30 Sekunden andauernde Ruhephase. Während beider, der Stimulations- und der Ruhephase, wurde am linken Zeigefinger die CPT (Wahrnehmungs-Reizschwelle) gemessen.

Durch die Elektrode am linken Zeigefinger wurde mit einer Frequenz von $1 \mathrm{~Hz}$ mit dem Digitimer stimuliert. Begonnen wurde die Stimulation mit 0 Volt und 
anschließend schrittweise um 1-Volt-Schritte erhöht. Die Spannung wurde schrittweise gesteigert, bis der Proband berichtete, ein Gefühl, also eine Art Sinneseindruck, wahrzunehmen. Wichtig war es, dass die Probanden sich immer die gleiche Art der Sinnesempfindung merkten als ihren persönlichen WahrnehmungsBeginn. Anschließend wurde die Stromstärke wieder herunter gedreht. Die Zeitdauer von Beginn der Stimulation bei 0 Volt bis zum Bemerken der Stimulation vom Probanden sollte 30 Sekunden nicht überschreiten. Hat man die Reizschwelle relativ schnell innerhalb der jeweiligen Phase bestimmt, so wurde bis zum Ablauf der Phase gewartet, bis die 30 Sekunden wieder abgelaufen waren. Dieser Ablauf wurde $8 \mathrm{mal}$ wiederholt und dauerte 8 Minuten an. Um die Ergebnisse der Versuche zur Bestimmung der CPT, während und ohne Stimulation, mit der Analyse der BOLDSignal-Änderungen vergleichen zu können, wurde die prozentuale Änderung der Empfindung von Reizschwellen während simultaner, contralateraler Stimulation im Vergleich zum Nicht-Stimulations-Zustand anschließend berechnet und dargestellt (\%-Änderung: zwischen Stimulation und Nicht-Stimulation).

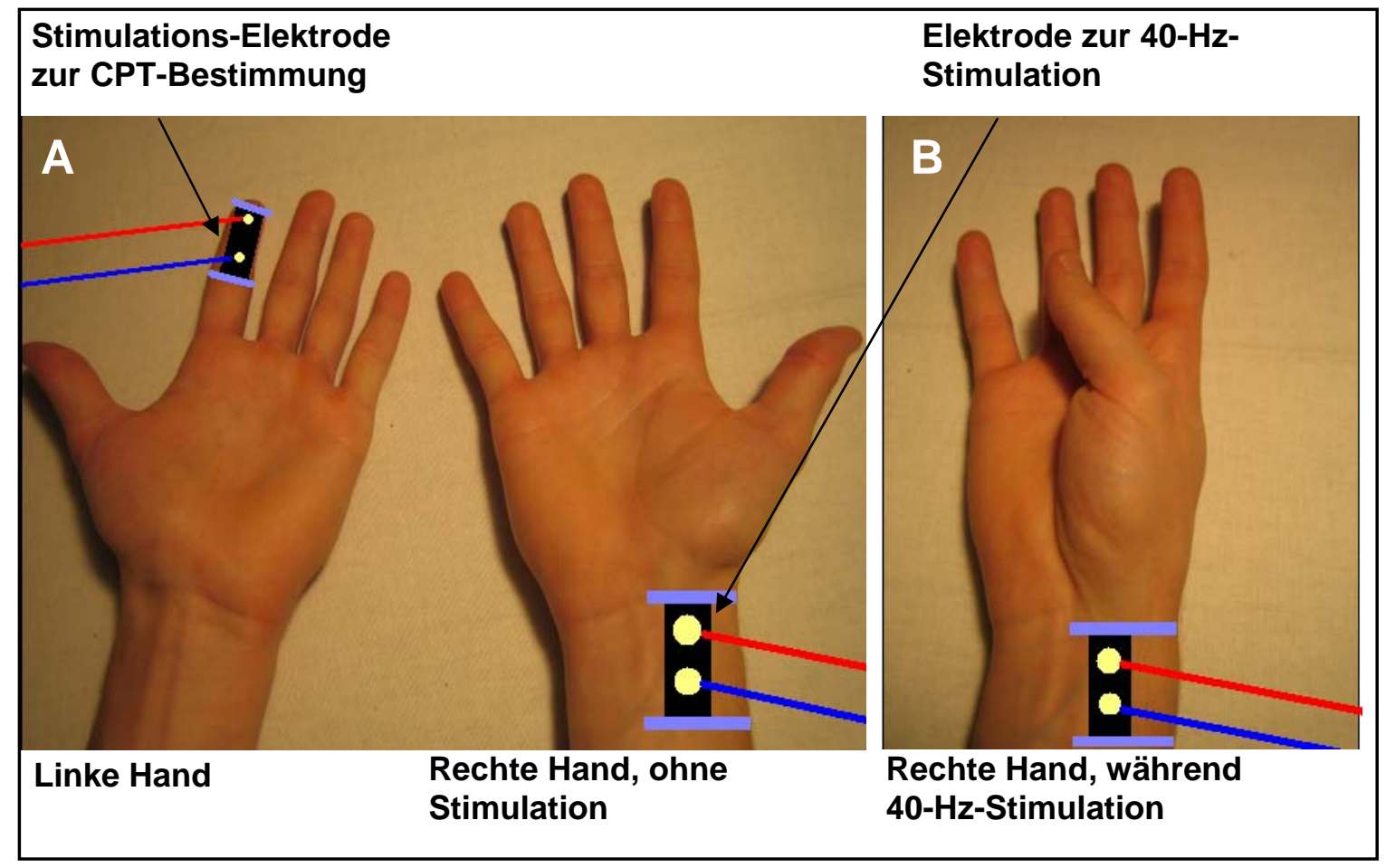

Abbildung 17: Dargestellt ist der Versuchsaufbau der psychophysiologischen Messungen. B zeigt die rechte Hand mit Kontraktion der vom N. medianus innervierten Thenar-Muskulatur während der 40-Hz-Stimulation. An der linken Hand war keine Kontraktion zu sehen. 


\section{3.) Ergebnisse}

\subsection{Hämodynamische BOLD-Antwort beim FT}

Um zu zeigen, wie die hämodynamische Antwort bei den älteren Probanden im Vergleich zu den jüngeren aussieht, ließen wir beide Altersgruppen das FT durchführen. Wir ließen die Probanden motorisch aktive Aufgaben ausführen im Vergleich zur passiven $40-\mathrm{Hz}$-Stimulation. Beim aktiv induzierten Gegenüberstellen der Finger werden neben dem sensomotorischen Kortex, weitere ZNS-Areale aktiviert und können anschließend im fMRT analysiert und verglichen werden. Es ist interessant zu wissen, ob und in wie fern sich eventuell vorhandene altersbedingte Unterschiede darstellen und sich ein Zusammenhang mit BOLD-Signal-Änderungen aufzeigen lässt. Im Fokus unseres Interesses stand die Frage, ob die älteren Probanden eine schlechtere, gleiche oder bessere hämodynamische Antwort zeigen. Bis auf den contralateralen SI-Kortex zeigt die ältere Gruppe wie in den Abbildungen 18 und 19 ersichtlich in den von uns fokussierten Kortexarealen eine zum Teil stärkere PBR als die jüngere. Die älteren Probanden aktivieren also nicht wesentlich schlechter, sondern mit einer zum Teil höheren Amplitude der BOLD-SignalÄnderung ins Positive. Die jüngeren Probanden weisen beim Ausführen der gleichen motorischen Aufgabe, außer im contralateralen SI, weniger starke PBR auf. Die hämodynamische Antwort der älteren Gruppe ist somit nicht unbedingt schlechter, sondern entweder gleichwertig (SII) oder sogar deutlicher ins Positive (SI ipsilateral) als bei der jüngeren. 


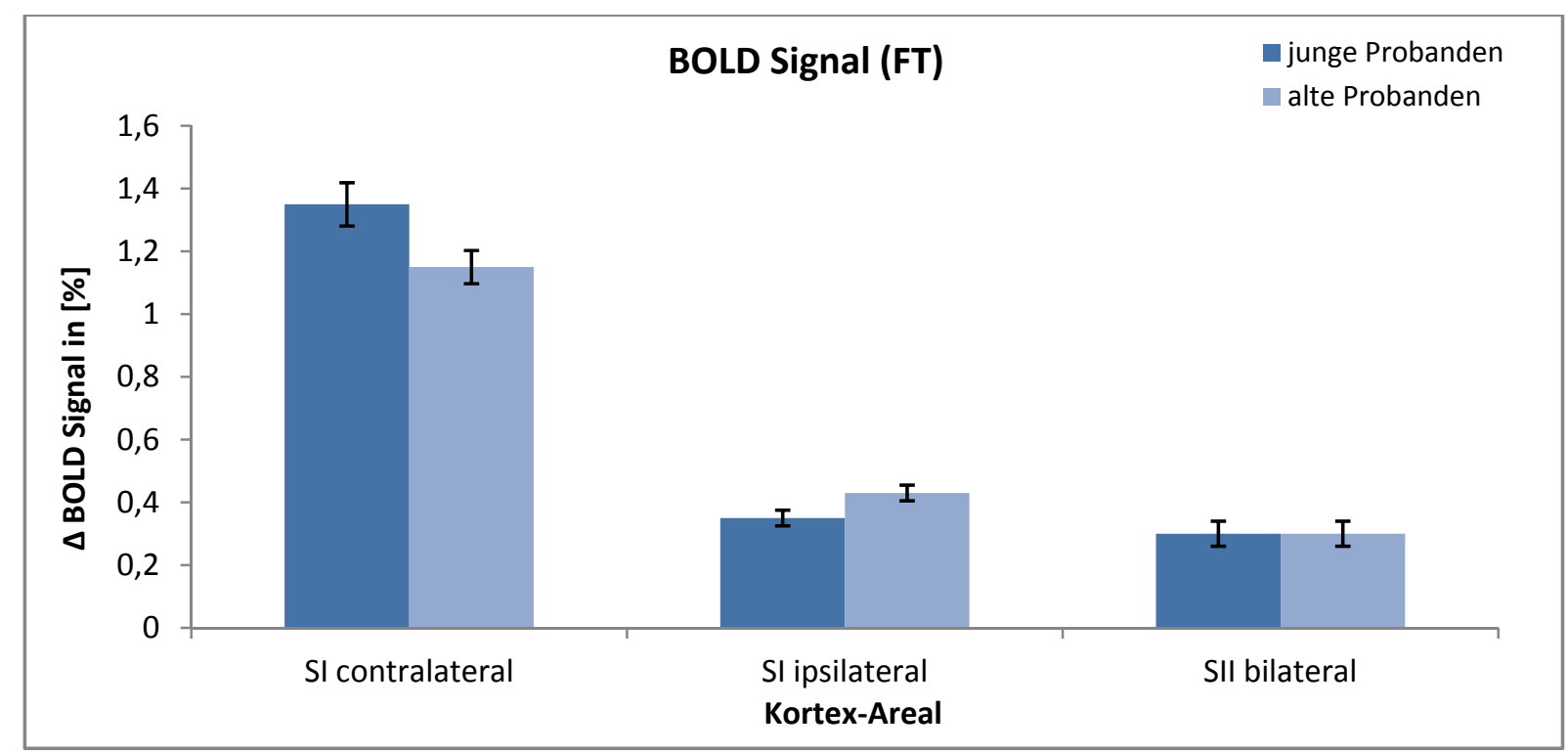

Abbildung 18: BOLD-Antworten (Maximalwerte) im SI- und SII-Kortex in beiden Altersgruppen mit Standardabweichung (SD).

Bei der Betrachtung weiterer von uns ausgewählter Kortexareale, wie zum Beispiel dem ipsilateralen Cerebellum, contralateralen Thalamus und Putamen wird deutlich, dass die hämodynamische Antwort bei den älteren Probanden nicht geringer ausfällt als bei den jüngeren, sondern positiver (Abbildung 19).

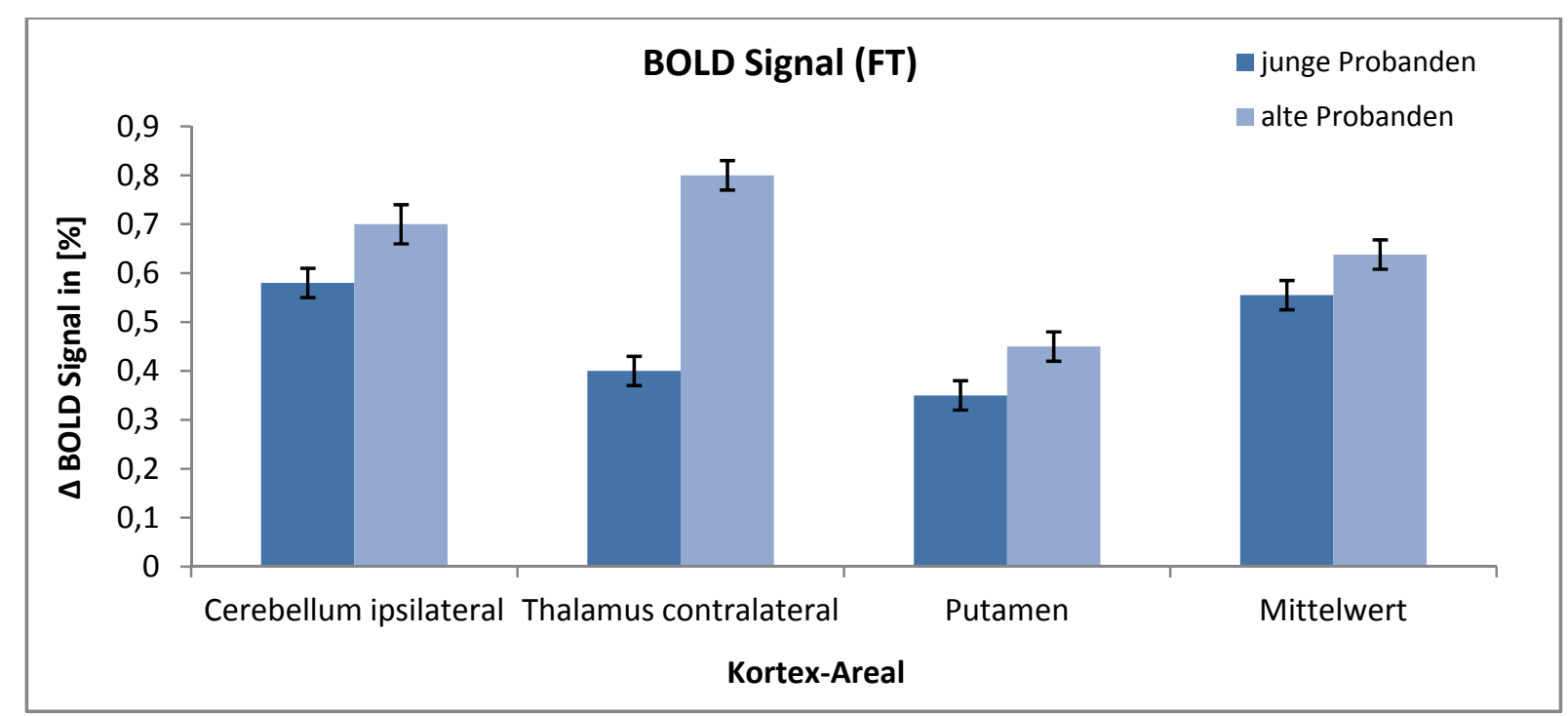

Abbildung 19: BOLD-Antworten (Maximalwerte) in weiteren betrachteten Kortexarealen mit Standardabweichung (SD) und dem Mittelwert der Kortexareale aus den Abbildungen 18 und 19. 
Die Aktivierungen sind bei den älteren Probanden in den von uns fokussierten Kortexarealen nicht nur oft stärker ins Positive, sondern sie umfassen auch, wenn man die Aktivierungskarten beider Altersgruppen betrachtet, mehr Kortexareale als bei den jüngeren. Es werden für die gleiche motorische Aufgabe bei der älteren Gruppe mehr Kortexareale rekrutiert als bei der jüngeren (Abbildungen 20 und 21).

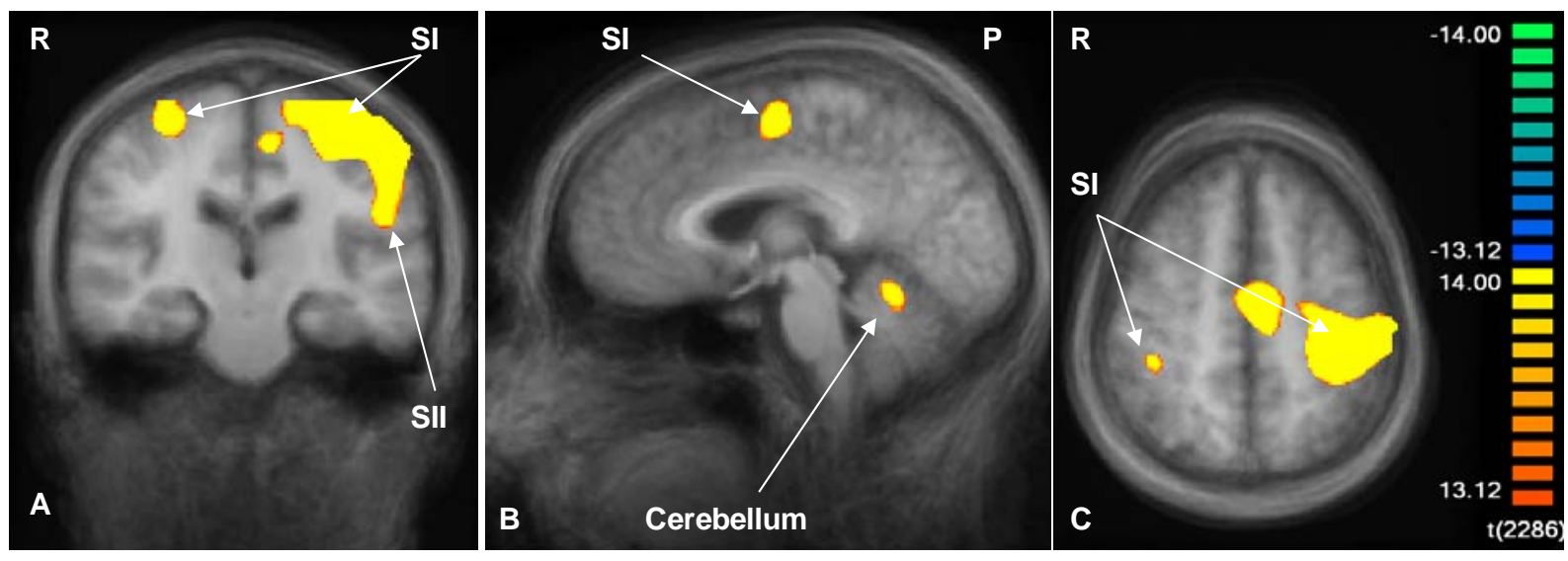

Abbildung 20: Gruppenkarte der jungen Probanden mit einer PBR im SI-und SII-Kortex sowie dem Cerebellum.
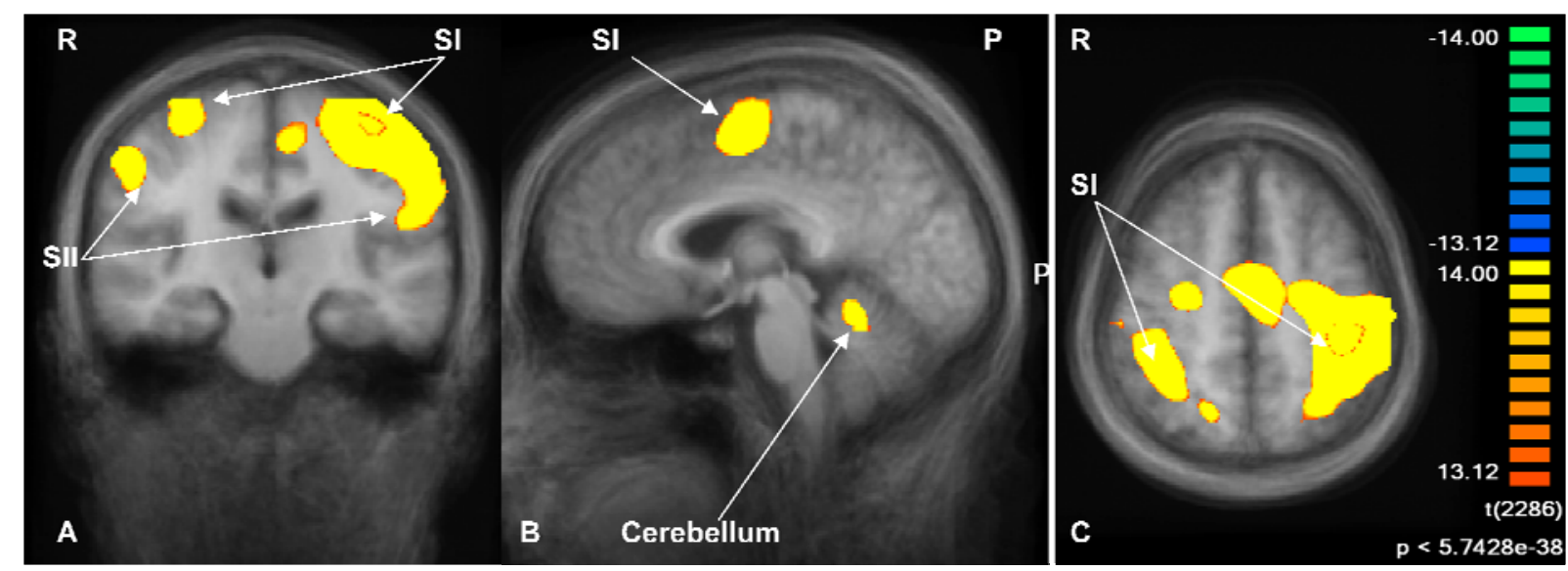

Abbildung 21: Gruppenkarte der alten Probanden mit einer PBR im SI- und SII-Kortex sowie dem Cerebellum. Die Aktivität ist ausgeprägter, mehr Kortexareale umfassend und sie zeigt eine stärkere PBR im Vergleich zu den jüngeren Probanden.

Neben den Aktivierungskarten spiegelt sich der Effekt, dass die älteren Probanden nicht schlechter, sondern mindestens gleichwertig aktivieren wie die jüngeren, auch in den Zeitverläufen des SI-MI- und MI-Kortex wider (Abbildung 22). Der Zeitverlauf der jungen und alten Probanden verläuft im contralateralen SI-MI-Kortex (A) sehr ähnlich. Steigung und Maxima der Kurven scheinen sich fast zu gleichen. Bei den jüngeren Probanden ist ein leicht erhöhtes und Zeit versetztes Maximum zu 
erkennen. Ebenso im ipsilateralen SI-MI-Kortex (B) verlaufen die Kurven sehr ähnlich. Lediglich das Maximum der jüngeren Probanden ist leicht Zeit versetzt. Im ipsilateralen MI-Kortex (C) wird deutlich, dass die ältere Gruppe eine höhere PBR aufweist. Die Feststellung, dass sich die Kurvenverläufe im ipsilateralen SI-MI sehr ähneln, ist besonders im Hinblick auf die Bewertung der 40-Hz-Ergebnisse später interessant.

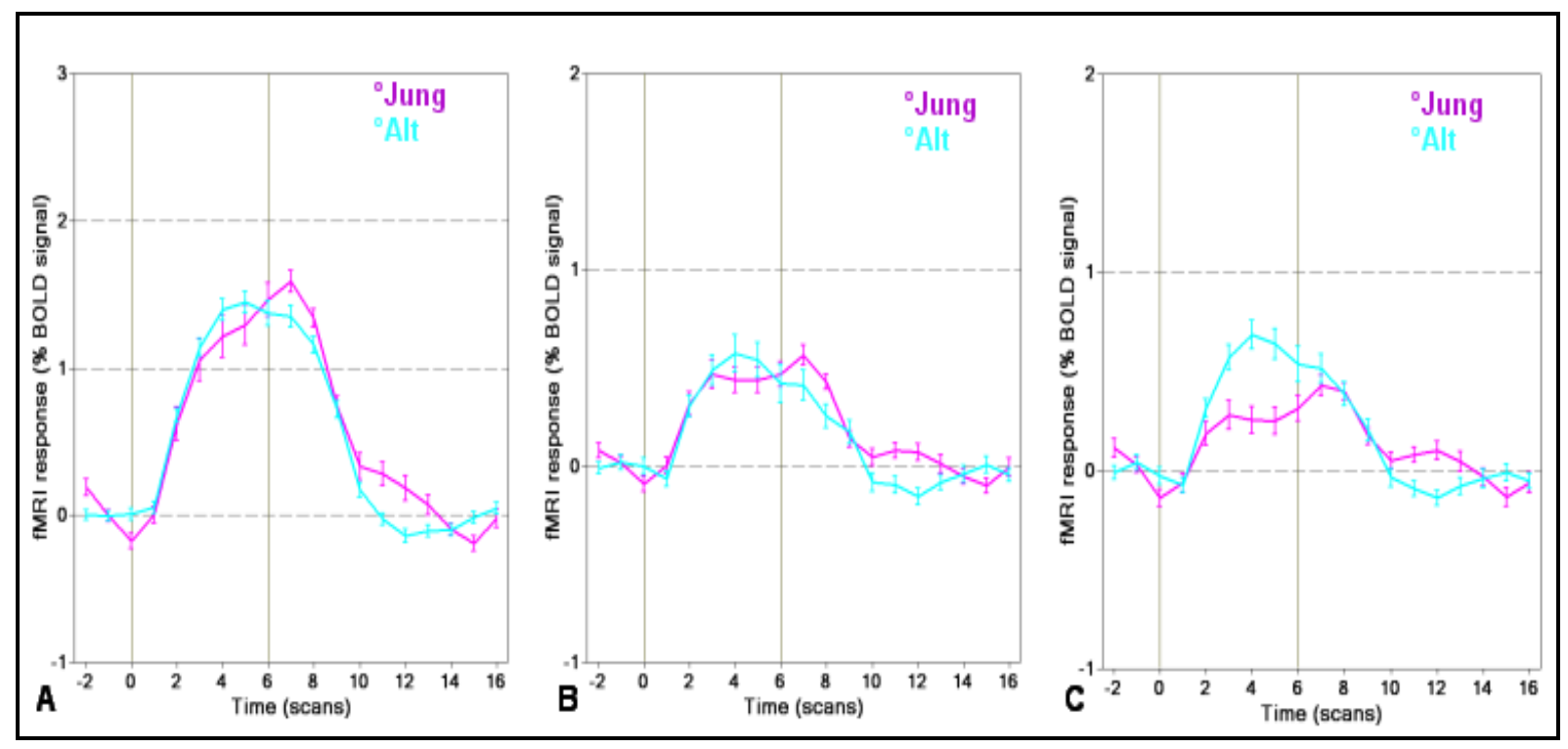

Abbildung 22: Zeitverläufe der jungen und alten Probanden im contralateralen SI-MI- (A), ipsilateralen SI-MI- (B) und im ipsilateralen MI-Kortex (C) mit Standardfehler.

\subsection{BOLD-Antworten der sensorischen Stimulation mit $40 \mathrm{~Hz}$}

Während der elektrischen Stimulation des rechten N. medianus wurden in beiden Altersgruppen bekannte sensomotorische Kortexareale aktiviert. Dazu zählten der contralaterale SI-Kortex, beidseitig das parietale Operculum (SII-Region), die contralaterale Insel und der contralaterale Thalamus. Im primären sensorischen Kortex (SI), ipsilateral zur peripheren Stimulation, stellten wir beim Vergleichen beider Altersgruppen fest, dass es überwiegend in der Gruppe der jungen Probanden zu deutlicher kortikaler Deaktivierung mit einer negativen BOLD-Signal-Antwort kam. In einer Einzelprobanden-Analyse der jungen Gruppe stellten wir fest, dass 12 von 14 Studien-Teilnehmern (86\%) das beschriebene Aktivitätsverhalten im ipsilateralen SI zeigten. Zeitverlaufs-Analysen der negativen Antwort machten eine transiente, kurzzeitige Abnahme des BOLD-Signals zu Stimulus-Beginn deutlich, welches langsam zum Grundlinien-Status am Ende der Stimulation zurückkehrte. In beiden Altersgruppen beobachteten wir auf der contralateralen Seite des Weiteren 
Aktivierungen im Bereich des Gyrus precentralis und leichte BOLD-Signal-Abfälle im Precuneus. Diese waren ausgeprägter bei den jungen Probanden vorhanden. Zusätzlich beobachteten wir in beiden Altersgruppen auf der ipsilateralen Seite eine deutliche Aktivierung des Cerebellums. Zu beachten ist im ipsilateralen SI-Kortex die NBR bei der jungen Altersgruppe. Im Gegensatz dazu zeigen die alten Probanden in diesem Areal eine weniger starke NBR (siehe dazu auch Abbildung 26 und Tabellen 01, 02). Die jungen Probanden zeigen eine stärkere PBR in weiteren Kortexarealen, wie zum Beispiel im contralateralen SI, SII und ipsilateralen SII (Abbildung 23).

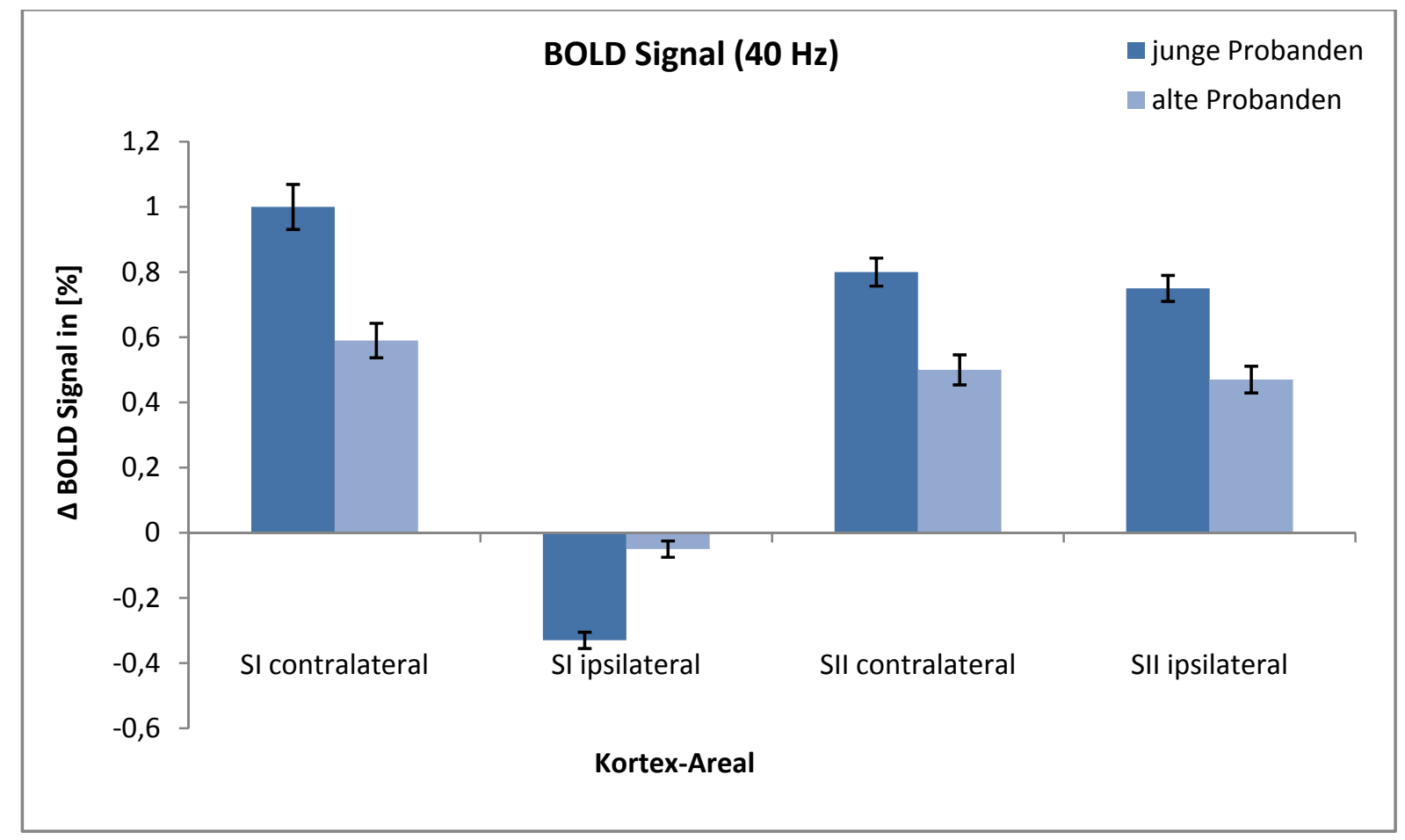

Abbildung 23: BOLD-Antworten (Maximal- bzw. Minimalwerte) im SI- und SII-Kortex, ipsilateral und contralateral zur Stimulation des rechten N. medianus mit Standardabweichung (SD). 


\subsubsection{Analyse des SI-Kortex}

Einen genaueren Fokus legten wir bei der Analyse der BOLD-Antworten auf den SIKortex (Abbildung 24). Bei Stimulation des rechten N. medianus zeigen die jüngeren Probanden in der Gruppenkarte (A, B, C) eine PBR auf der contralateralen Seite sowie eine NBR auf der ipsilateralen Seite. Die älteren Probanden (D, E, F) zeigen eine PBR auf der contralateralen Seite und keine markante NBR im ipsilateralen SIKortex im Vergleich zur jungen Gruppe.
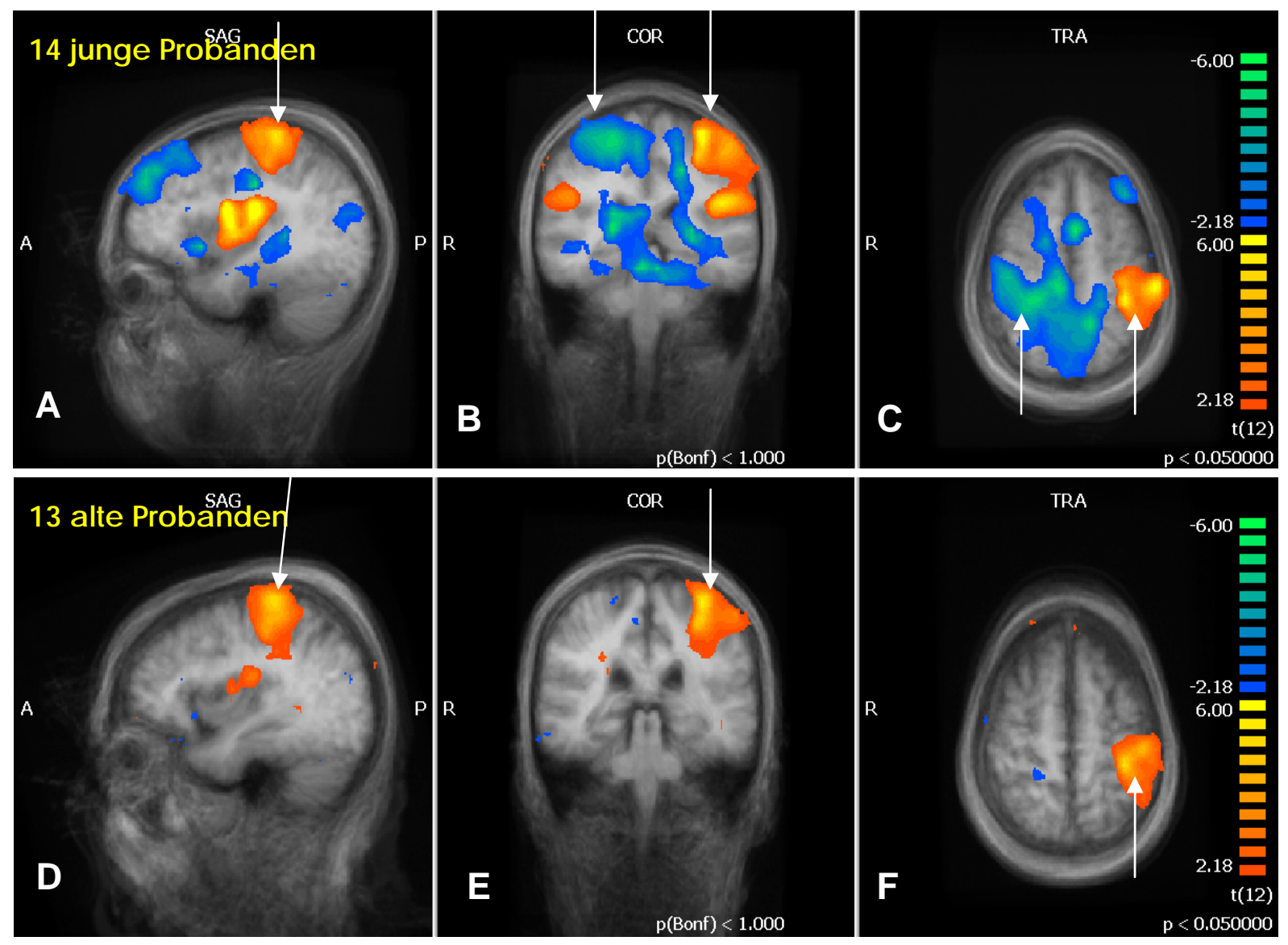

Abbildung 24: Schnitte mit Darstellung der jungen und alten Aktivitäts-Gruppenkarten des SI-Kortex. In A-C ist die Gruppe der jungen Probanden dargestellt, in D-F die Gruppe der alten Probanden. Die jüngeren Probanden zeigen im ipsilateralen SI-Kortex eine deutlichere NBR, im Gegensatz zu den älteren Probanden.

Im Zeitverlauf stellen sich die SI-Kortexareale der beiden Altersgruppen unterschiedlich dar. Beide zeigen auf der contralateralen Seite (Abbildung 25) eine initiale PBR, die schrittweise zurückgeht und sich gegen Stimulations-Ende mit einem zweiten Anstieg darstellt. Die PBR der jüngeren Probanden zeigt ein deutlich höheres Maximum als das der älteren Gruppe. Ebenso eine stärke Steigung der 
BOLD-Signal-Änderung ins Positive, kurz nach Stimulations-Beginn. Der Abfall der PBR stellt sich bei den jüngeren Probanden ebenfalls steiler da. Es fällt in einer kürzeren Zeitspanne stärker ab, allerdings nicht auf den Ausgangswert bei Null. Der abschließende Anstieg des BOLD-Signals nach ca. 15 Sekunden fällt bei der jüngeren Altersgruppe ebenfalls steiler, früher und mit einem höheren Maximum aus. Anschließend fallen beide Kurven zum Stimulations-Ende wieder ab.

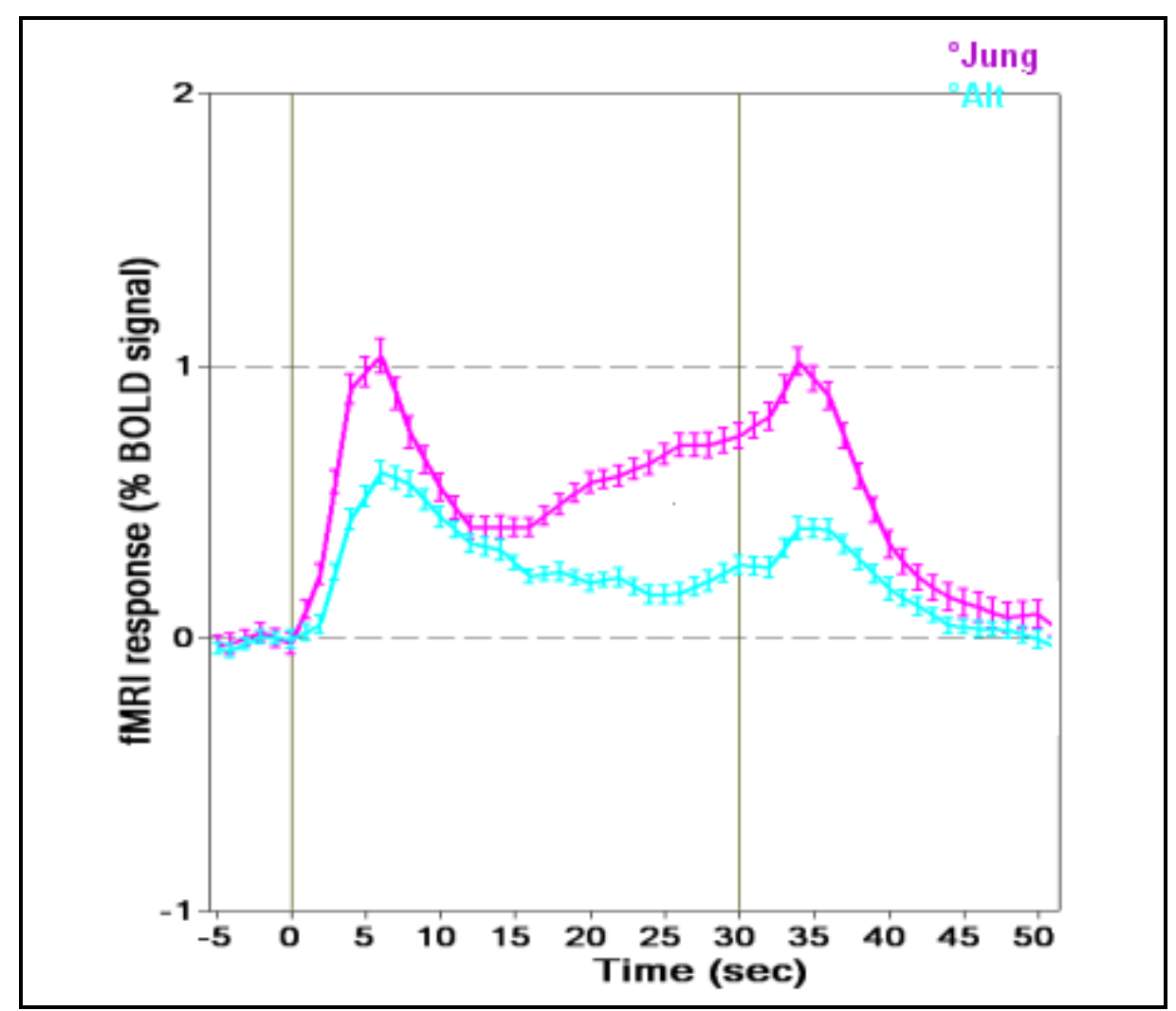

Abbildung 25: Zeitverlauf der BOLD-Antworten im contralateralen SI-Kortex der jüngeren und älteren Probanden mit Standardfehler.

Am Zeitverlauf des BOLD-Signals im ipsilateralen SI-Kortex (Abbildung 26) wird eine stärkere NBR der jüngeren Altersgruppe deutlich. Negative Steigung und Minimum fallen deutlich größer aus als bei der älteren Gruppe. Die Kurve der älteren Gruppe pendelt um die Grundlinie, mit leichten Ausschlägen ins Positive oder Negative. 


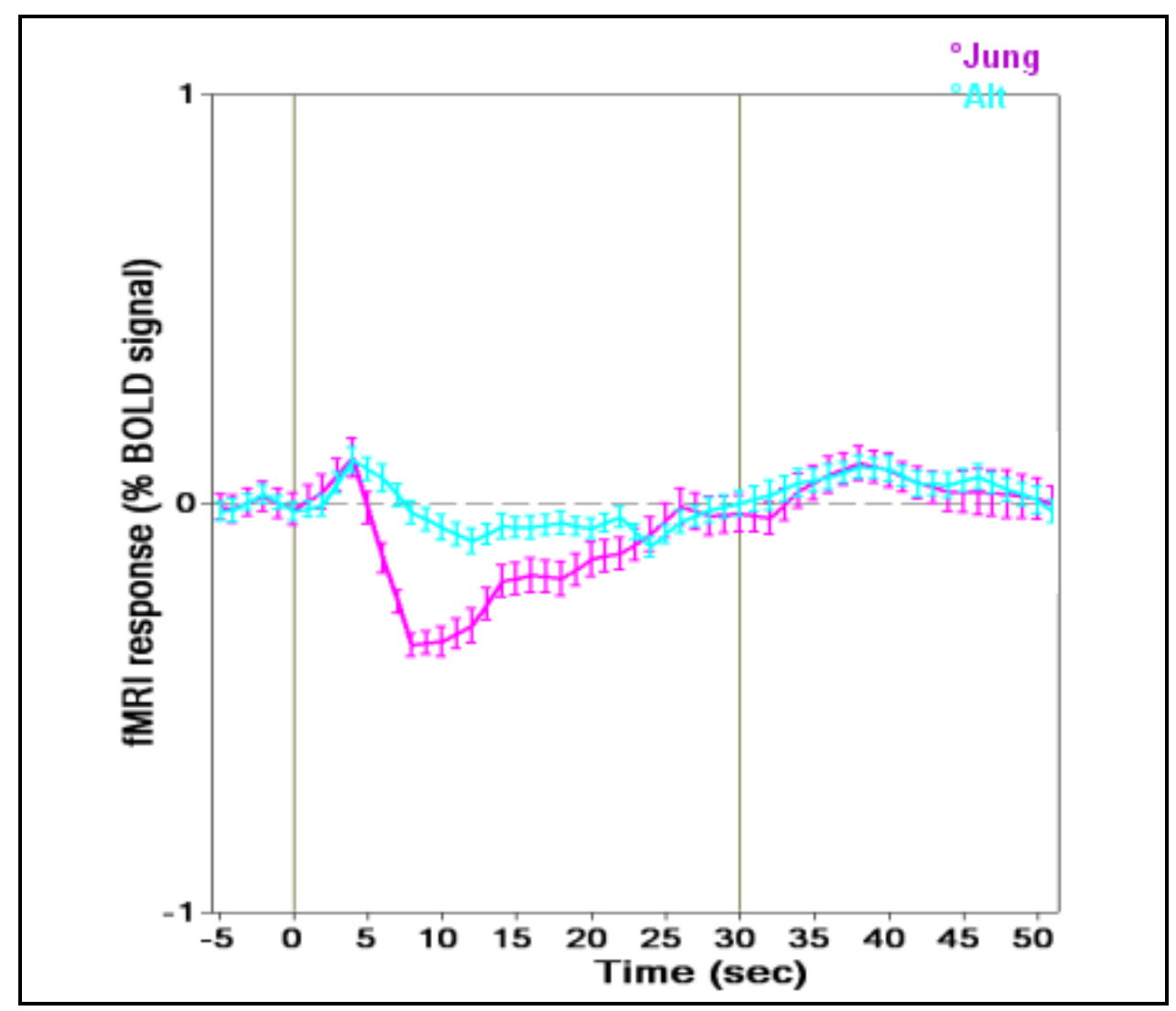

Abbildung 26: Zeitverlauf der BOLD-Antworten im ipsilateralen SI-Kortex der jüngeren und älteren Probanden mit Standardfehler.

\subsubsection{Analyse des SIl-Kortex}

Als weiteres Areal untersuchten wir die Aktivitätsunterschiede im SIl-Kortex. Die Sagittalschnitte (Abbildung 27) und die dazugehörigen Zeitverläufe zeigen die Aktivierung vom SII-Kortex, bilateral, unter Stimulation des rechten N. medianus. Beide Gruppen zeigen eine PBR im contralateralen und ipsilateralen SII-Kortex. Im Vergleich von jungen und alten Probanden stellt sich die PBR im SIl der alten Probanden im Verlauf ähnlich wie die der jungen Gruppe da. Das Signal ist im Vergleich zu den PBR-Werten der jüngeren Probanden jedoch abgeschwächter. Im Kurvenverlauf gleichen sich Steigungs-Periode, Maximumszeitpunkt und Abfallzeit der PBR. Es lässt sich zusammenfassend erkennen, dass sich die Signal-Kurven der jungen und alten Altersgruppe im SII-Kortex, bilateral, relativ ähnlich verhalten. 


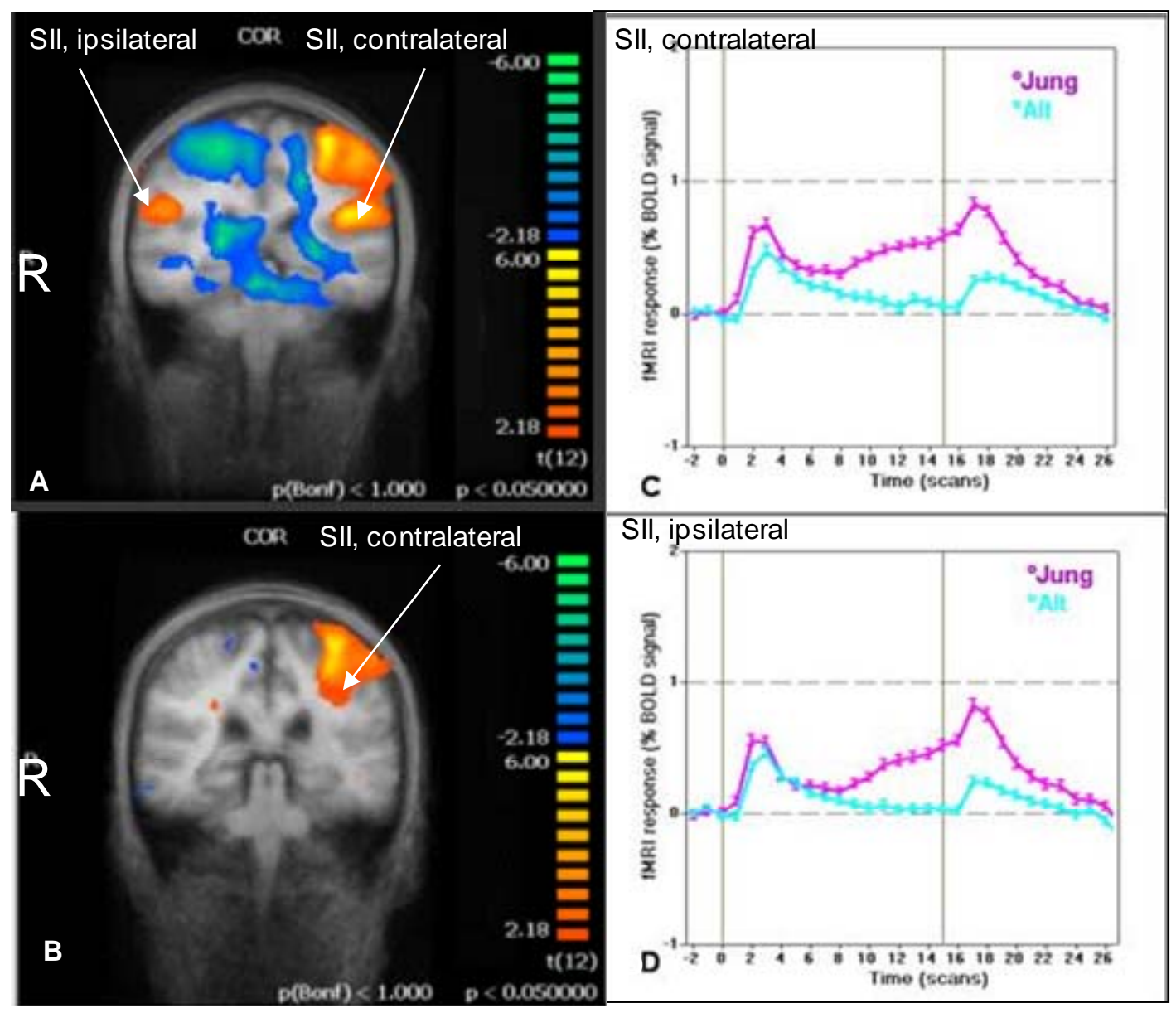

Abbildung 27: Dargestellt ist in A die PBR des SII-Kortex, bilateral auf der Aktivierungskarte der jungen Probanden, in B der alten Probanden auf der contralateralen Seite. C und D zeigen die Zeitverläufe der PBR im SII-Kortex mit Standardfehler. In C ist die PBR auf der contralateralen und in D auf der ipsilateralen Seite dargestellt mit Standardfehler. 


\subsubsection{Analyse weiterer Kortexareale}

Auffällig ist, dass es sich beim ipsilateralen Cerebellum bei unseren Messungen um eines der wenigen markanten Kortexareale handelt, das eine etwas höhere PBR bei den älteren Probanden aufzeigt (Abbildung 29), als bei den jüngeren (Abbildung 28). Sonst ist das BOLD-Signal in der jüngeren Gruppe, bezogen auf unsere ausgewählten Areale, durch eine stärkere PBR gekennzeichnet.

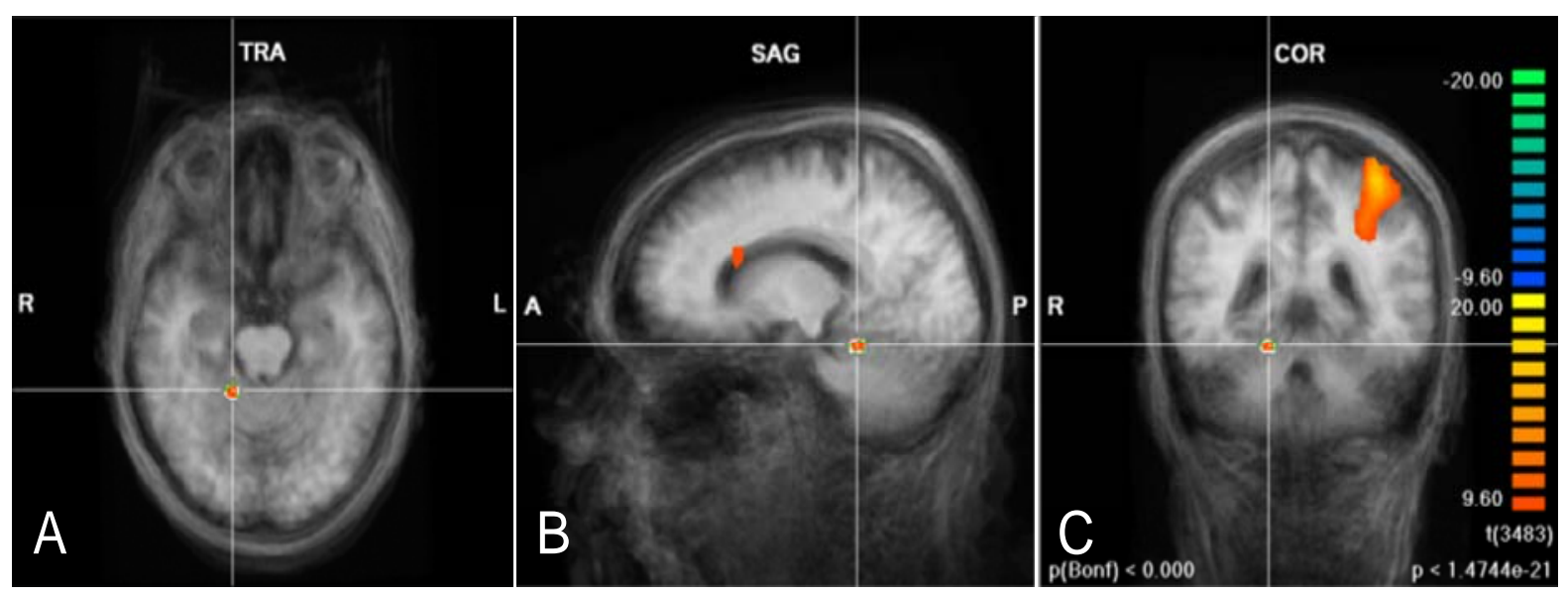

Abbildung 28: PBR im Cerebellum der jungen Gruppe auf der ipsilateralen Seite zur 40-HzStimulation des rechten $\mathrm{N}$. medianus.

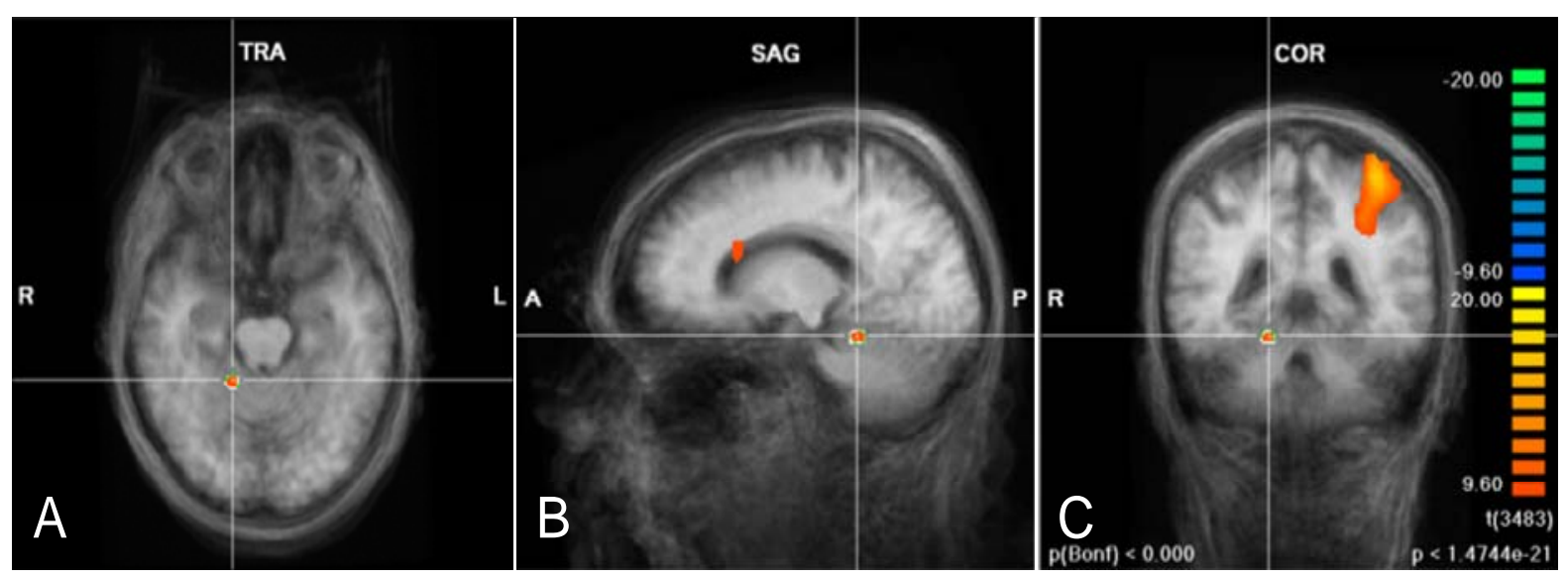

Abbildung 29: PBR im Cerebellum der alten Gruppe auf der ipsilateralen Seite zur 40-HzStimulation des rechten N. medianus.

Neben der Betrachtung der Aktivierungskarten wird dieser Effekt ebenfalls bei der Analyse der Zeitverläufe im ipsilateralen Cerebellum deutlich. Hier liegt das Maximum der Aktivität der älteren Probanden über dem der jüngeren (Abbildung 30). 


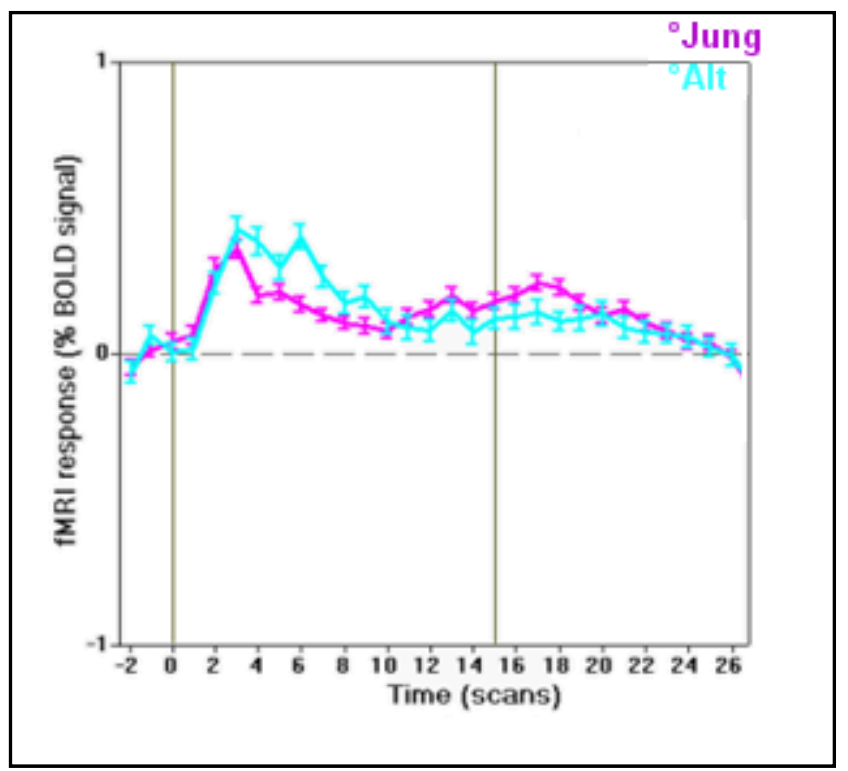

Abbildung 30: Zeitverläufe der BOLD-Signal-Änderungen im ipsilateralen Cerebellum der beiden Altersgruppen während der $40-\mathrm{Hz}$-Stimulation des rechten $\mathrm{N}$. medianus mit Standardfehler.

Die Zeitverläufe der BOLD-Signal-Änderung zeigt bei einer weiteren Analyse der Insel (Andere Lokalisation als Abbildung 27) bei den jüngeren Probanden ein höheres Maximum an (Abbildung 31).

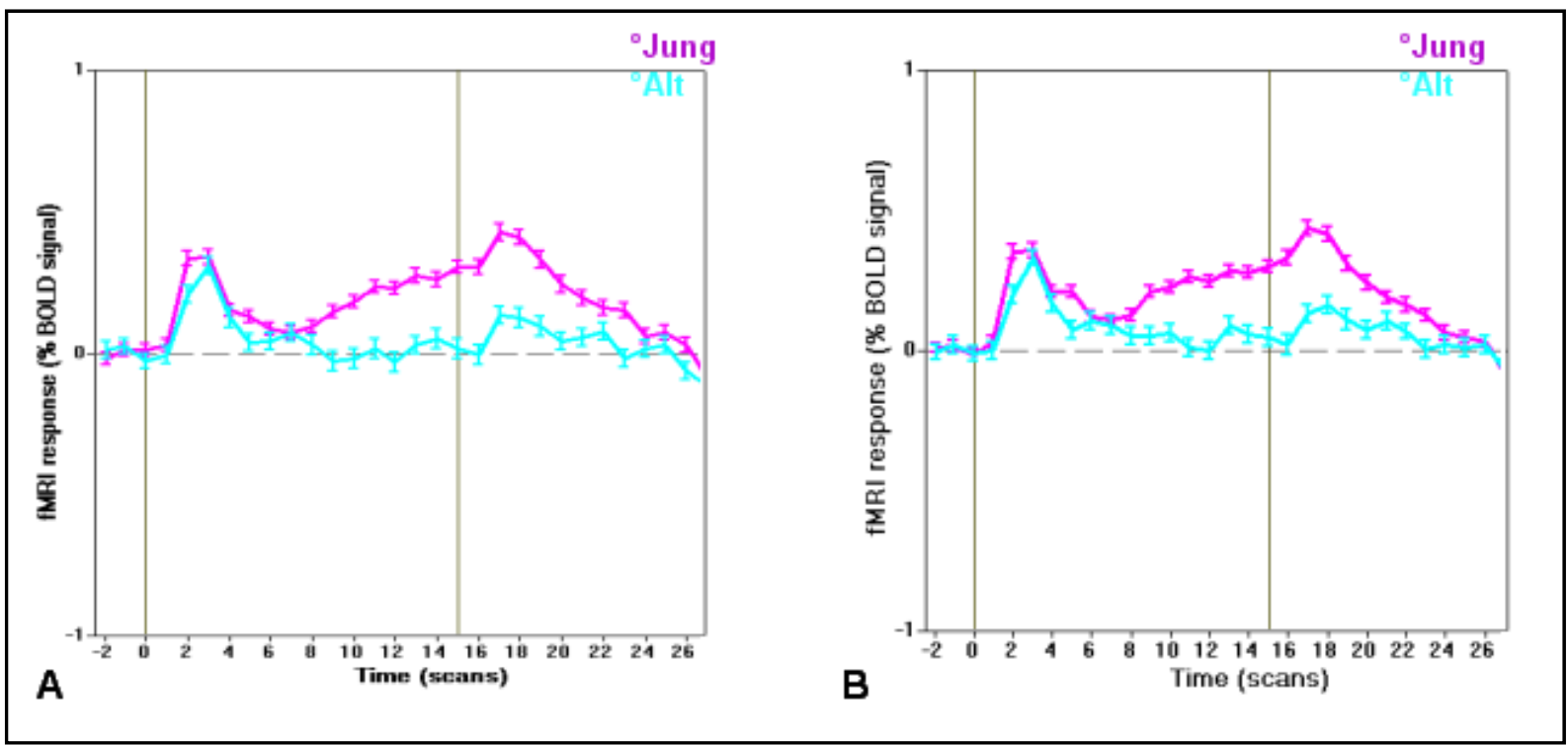

Abbildung 31: In A ist die PBR der Insel der jungen und alten Probanden-Gruppe auf der ipsilateralen Seite zur 40-Hz-Stimulation des rechten N. medianus mit Standardfehler dargestellt. In B ist die PBR der Insel der jungen und alten Probanden auf der contralateralen Seite mit Standardfehler dargestellt.

Des Weiteren zeigt die Analyse des contralateralen präzentralen und parasagittalen Kortex ein höheres Maximum ebenfalls bei den jüngeren Probanden (Abbildung 32). 


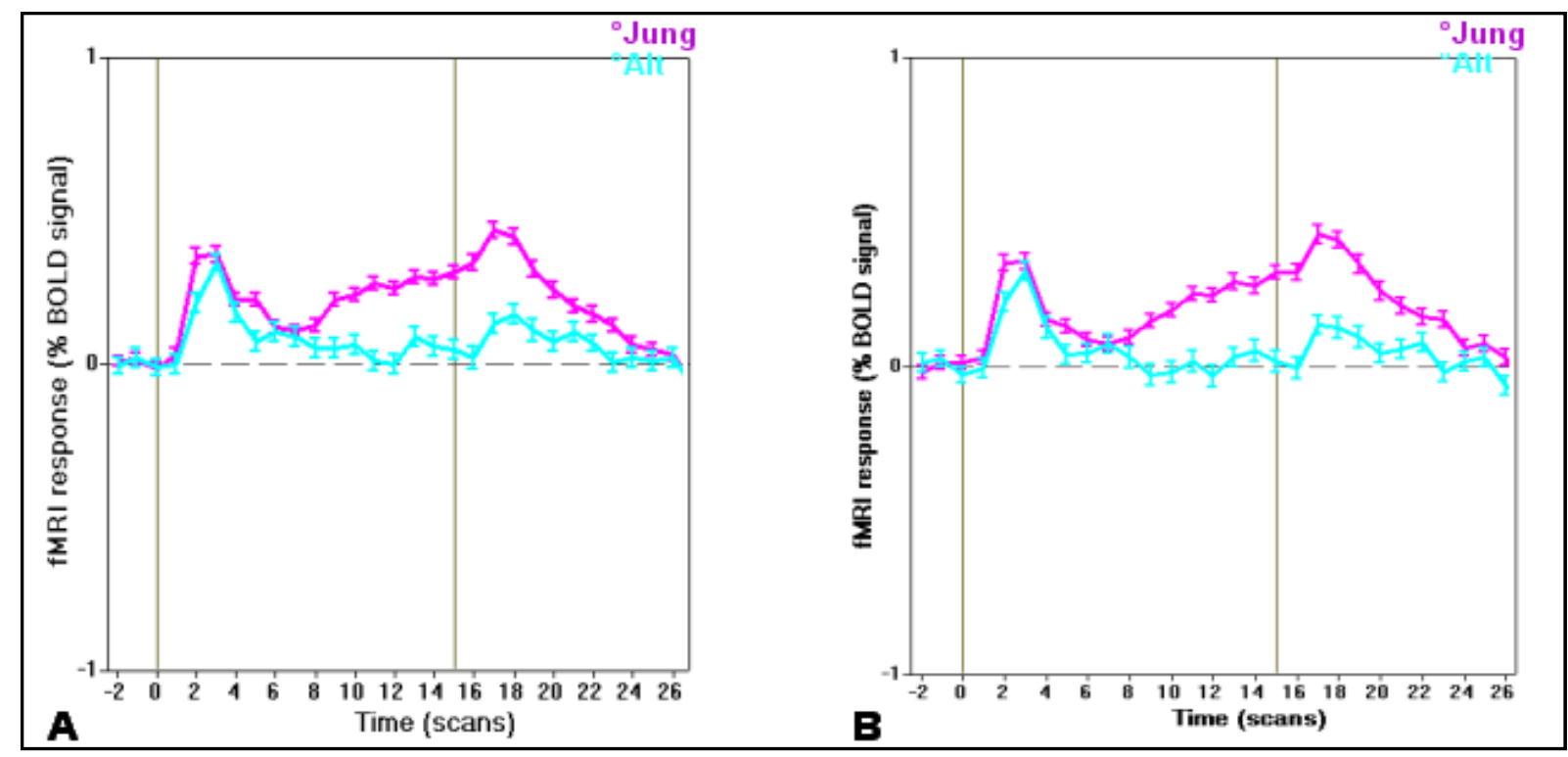

Abbildung 32: In A ist die PBR im präzentralen Kortex der jungen und alten Probanden-

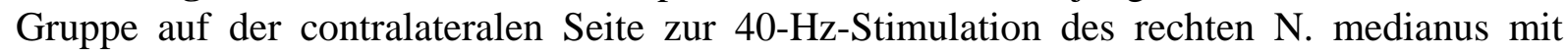
Standardfehler dargestellt. In B ist die PBR im parasagittalen Kortex der jungen und alten Probanden auf der contralateralen Seite dargestellt.

Die folgenden Tabellen stellen die Aktivierungen der $40-\mathrm{Hz}-S t i m u l a t i o n$ mit den dazugehörigen Talairach-Lokalisationen dar.

Tabelle 01: Darstellung der Gruppen-Aktivierungen mit den zugehörigen TalairachLokalisationen der jungen Probanden.

\begin{tabular}{|l|l|r|r|r|r|r|}
\hline Junge Probanden & BOLD & X & y & z & T-value & No Voxels \\
\hline Linke Hemisphäre & & & & & & \\
\hline SI & Pos & -37 & -31 & 55 & 14,96 & $>1000$ \\
\hline SII & Pos & -57 & -25 & 25 & 11,84 & 941 \\
\hline Cerebellum & Neg & -8 & -44 & -11 & $-6,33$ & 495 \\
\hline Thalamus & Pos & -15 & -19 & 5 & 9,15 & 347 \\
\hline Insula & Pos & -39 & 1 & 6 & 7,9 & 699 \\
\hline Areal 6 & Pos & -5 & -13 & 50 & 7,5 & 610 \\
\hline Putamen & Pos & -28 & -2 & 3 & 7,84 & 586 \\
\hline Rechte Hemisphäre & & & & & & \\
\hline SI & Neg & 38 & -27 & 57 & $-10,62$ & 417 \\
\hline SII & Pos & 55 & -25 & 22 & 8,9 & 666 \\
\hline Cerebellum & Pos & 13 & -44 & 14 & 6,39 & 177 \\
\hline Insula & Pos & 35 & -3 & 18 & 6,88 & 338 \\
\hline Thalamus & Neg & 18 & -20 & 9 & 6,22 & 550 \\
\hline
\end{tabular}

Bonferroni-Korrektur $\mathrm{p}<0.01$

Farbskala 5.14 - 8.00 
Tabelle 02: Darstellung der Gruppen-Aktivierungen mit den zugehörigen Talairachdaten der alten Probanden. Man beachte die Unterschiede im SI der rechten Hemisphäre zu den Jungen.

\begin{tabular}{|l|l|r|r|r|r|r|}
\hline Alte Probanden & BOLD & X & y & z & \multicolumn{1}{l|}{ T-value } & \multicolumn{1}{l|}{ No Voxels } \\
\hline Linke Hemisphäre & & & & & & \\
\hline SI & Pos & -37 & -31 & 55 & 12,43 & $>1000$ \\
\hline SII & Pos & -53 & -20 & 24 & 6,71 & 662 \\
\hline Thalamus & Pos & -16 & -17 & 4 & 6,87 & 282 \\
\hline Insula & Pos & -38 & -15 & 18 & 7,16 & 495 \\
\hline Areal 6 & Pos & -11 & -9 & 52 & 5,96 & 125 \\
\hline Rechte Hemisphäre & & & & & & \\
\hline SI & Neg & 35 & -32 & 59 & $-6,20$ & 189 \\
\hline SII & Pos & 54 & -24 & 24 & 6,16 & 371 \\
\hline Cerebellum & Pos & 13 & -43 & -12 & 7,50 & 389 \\
\hline
\end{tabular}

Bonferroni-Korrektur $\mathrm{p}<0.01$

Farbskala 5.14 - 8.00

\subsubsection{Gruppen-Analysen}

Die Subtraktionskarten zeigen die Aktivitätsunterschiede der beiden Altersgruppen im SI-Kortex in deutlicher Art und Weise auf. Zur Erklärung zeigt die erste Subtraktionskarte (Abbildung 33) die Kortexareale mit orange-gelber Färbung, bei denen die ältere Probandengruppe eine stärkere und damit positivere BOLD-SignalÄnderung aufwies als die jüngere Gruppe. Umgekehrt stehen die Kortexareale mit blau-grün Färbung für die Areale in denen die älteren Probanden ein schwächeres BOLD-Signal zeigten. Kortexareale bei denen sich das BOLD-Signal der jüngeren und der älteren Altersgruppe glichen, werden nicht farblich hervorgehoben und erscheinen grau. Beispielhaft wird hier der Focus auf den SI-Kortex gelegt und veranschaulicht, dass auf der contralateralen Seite das BOLD-Signal der älteren Gruppe schwächer ist und das der jüngeren Gruppe stärker ins Positive geht. Umgekehrt, auf der ipsilateralen Seite zur Stimulation des rechten N. medianus, wird ein stärkeres BOLD-Signal in der älteren Gruppe deutlich. Hier ist das BOLD-Signal der jüngeren Gruppe negativer. Zur Unterstützung werden die beiden zugehörigen Zeitverläufe für den ipsilateralen und contralateralen SI-Kortex mit dargestellt. 


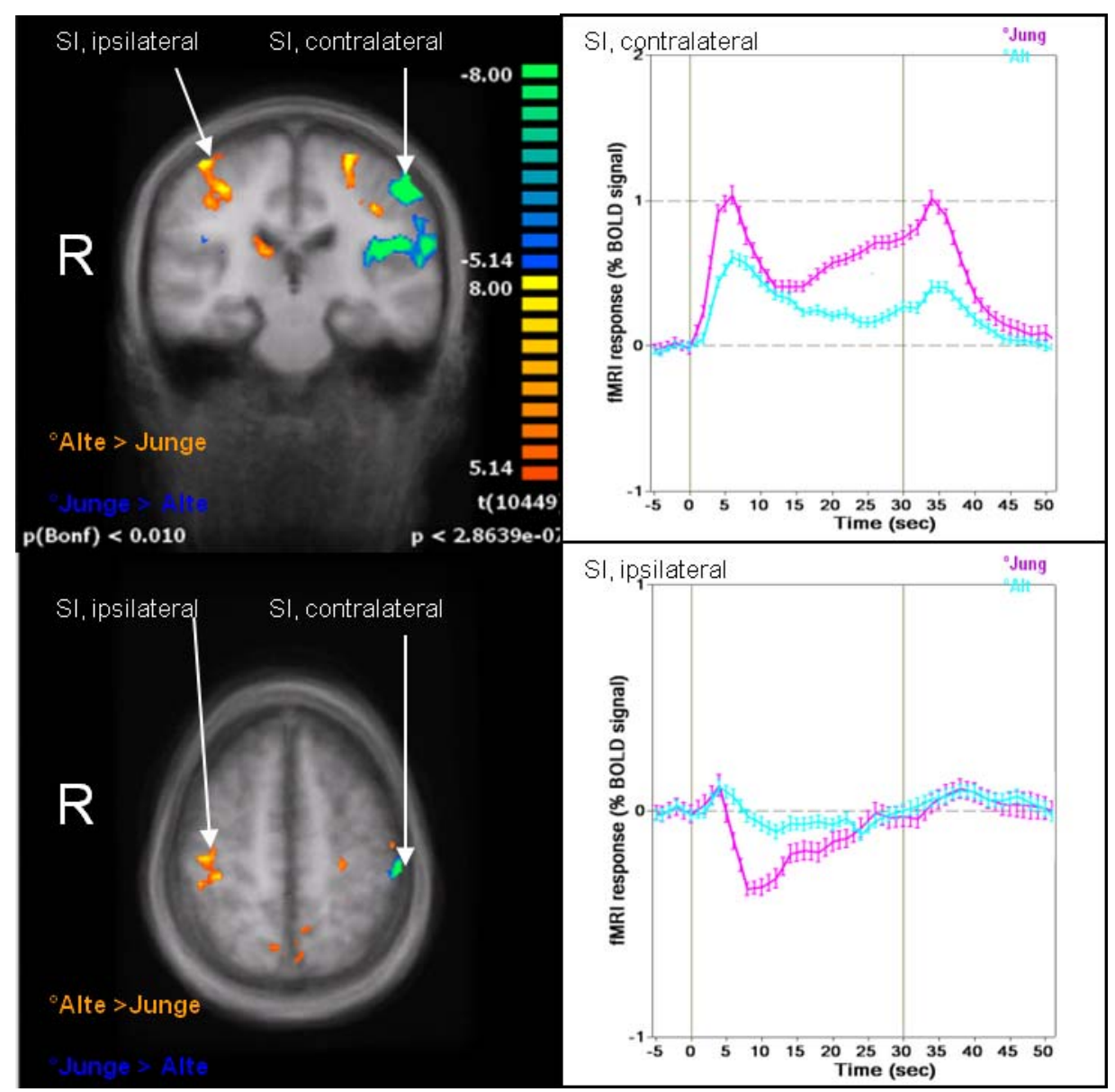

Abbildung 33: Subtraktionskarte der Gruppen-Analysen beider Altersgruppen. Man beachte die unterschiedliche Y-Achseneinteilung der beiden Zeitverlaufsachsen.

In der zweiten Subtraktionskarte (Abbildung 34) werden die Kortexareale in denen die jüngeren Probanden schwächere BOLD-Signal-Änderungen aufwiesen und dementsprechend schwächer aktivieren als die älteren in blauer Färbung dargestellt. SI und SII wurden hier veranschaulichend präsentiert. Im SI contralateral aktivieren die jüngeren Probanden stärker, im SI ipsilateral schwächer als die älteren. 


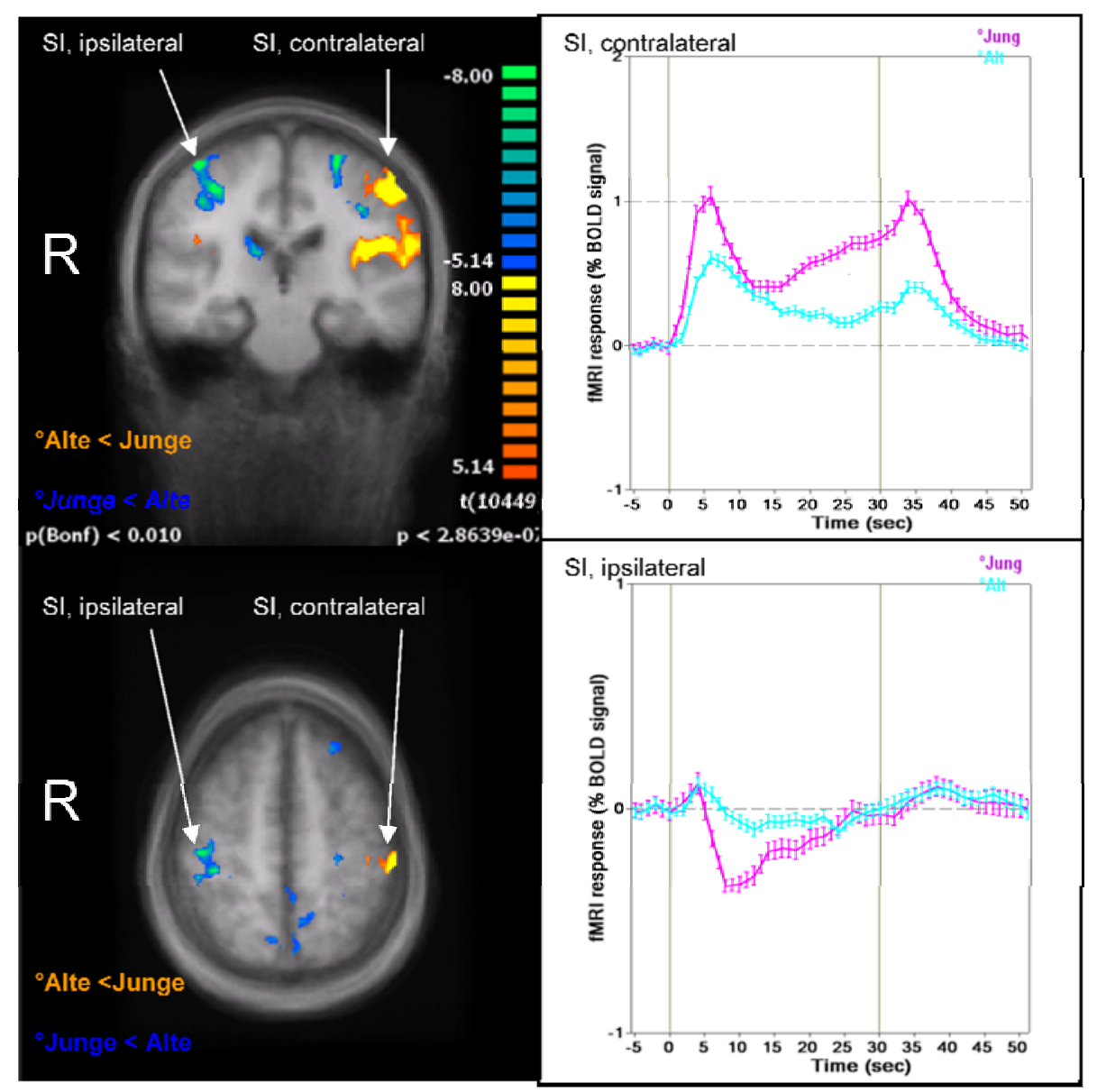

Abbildung 34: Diese Subtraktionskarte zeigt umgekehrt (zu Abbildung 33) in welchen Arealen die jüngere Probandengruppe stärker (orange-gelbe Färbung) aktivieren und damit mit einer positiveren BOLD-Signal-Änderung im Vergleich zur älteren Gruppe. Man beachte die unterschiedliche Y-Achseneinteilung der beiden Zeitverlaufsachsen.

\subsubsection{Einzelprobanden-Analysen}

Stellvertretend für die Gruppe der jüngeren Probanden ist in den folgenden Aktivierungskarten ein 23-jähriger, männlicher Proband dargestellt. Hier wird die NBR im SI-Kortex auf der ipsilateralen Seite deutlich aufzeigt (Abbildung 35) sowie die deutliche PBR im SI-Kortex auf der contralateralen Seite (Abbildung 36). Man beachte bei den Abbildungen 35 bis 38 die unterschiedlichen Y-Achseneinteilungen der Zeitverlaufachsen. 


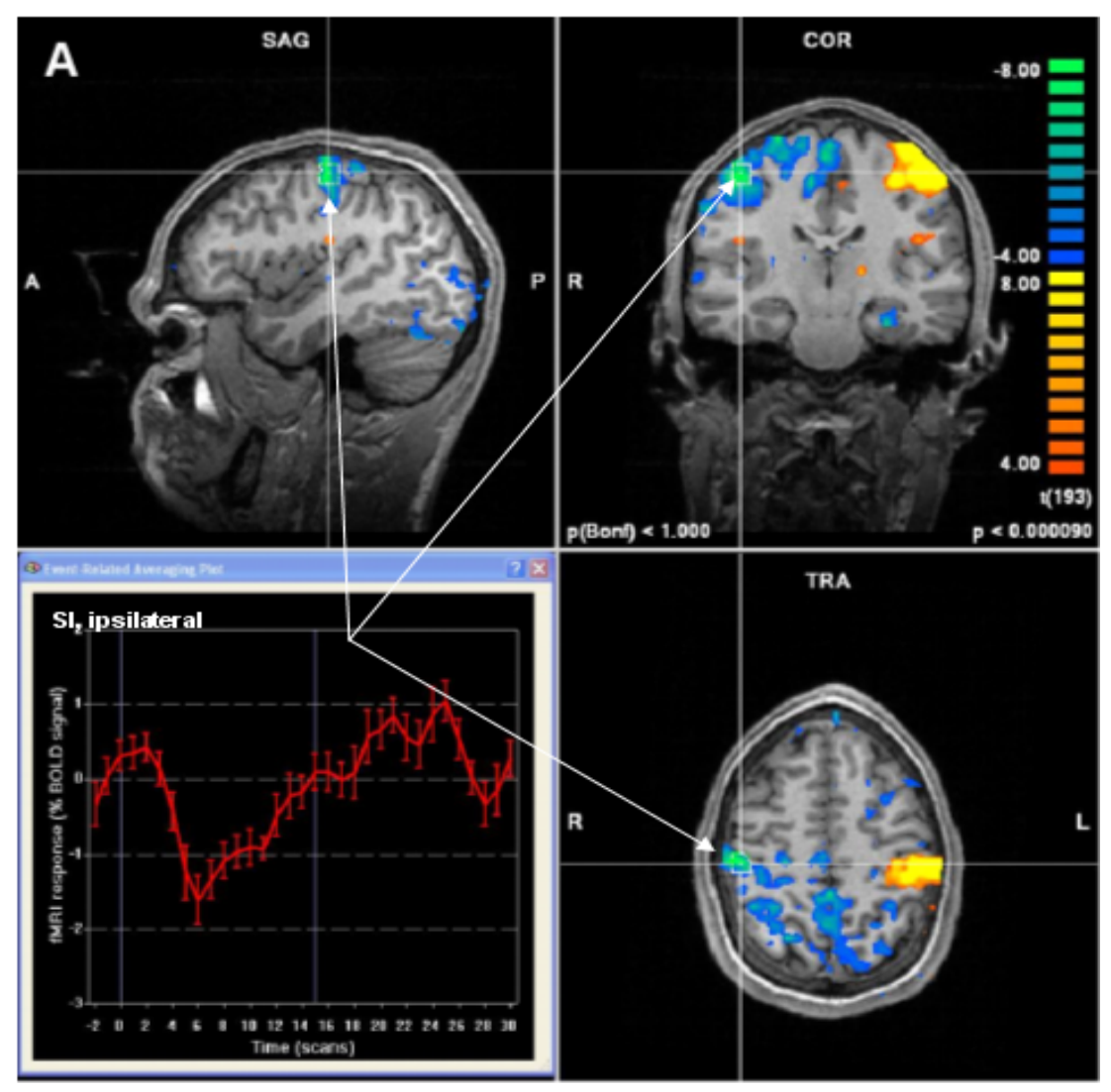

Abbildung 35: Junger Einzelproband mit Analyse der NBR im ipsilateralen SI-Kortex.

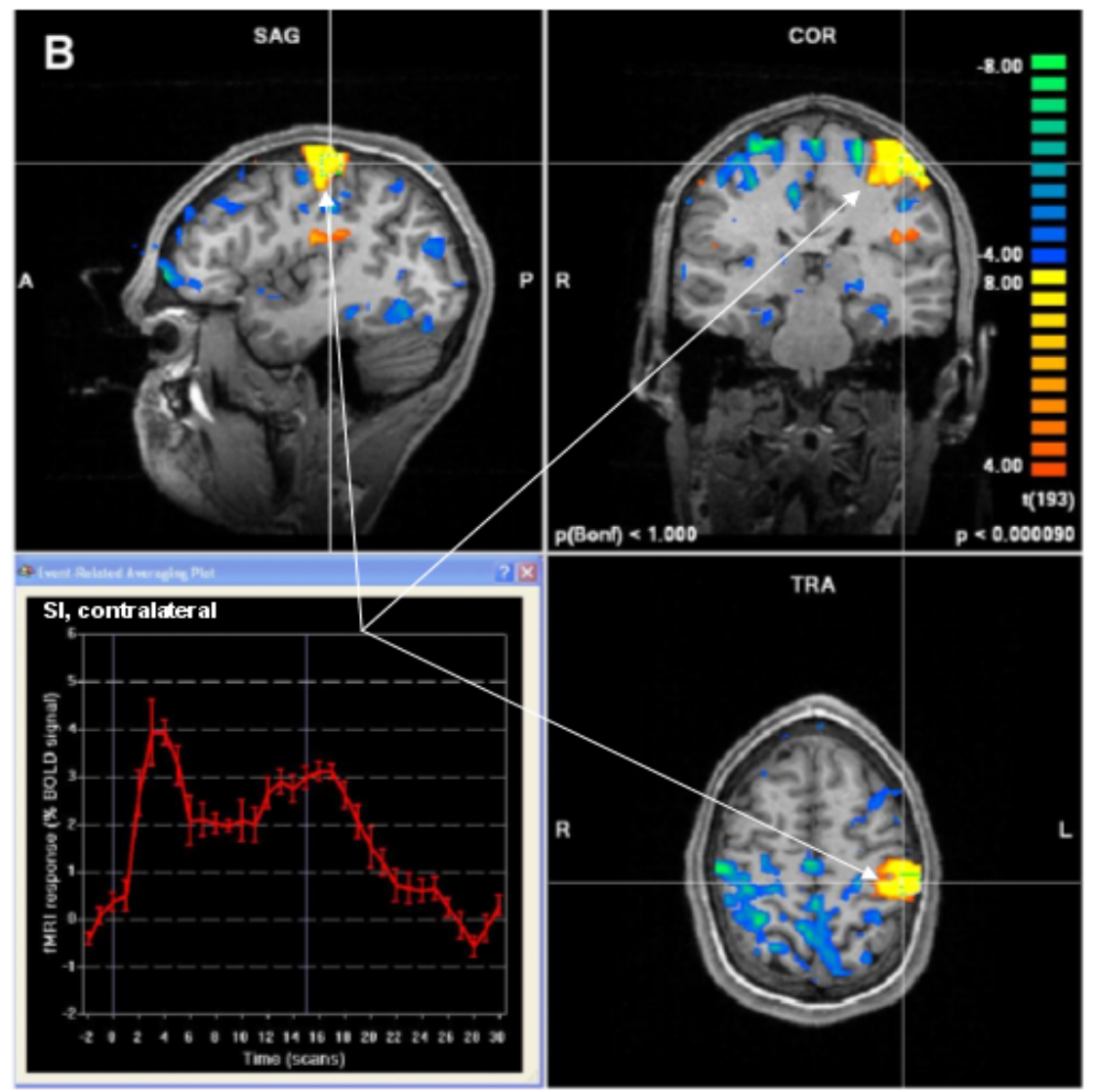

Abbildung 36: : Junger Einzelproband mit Analyse der PBR im contralateralen SI-Kortex. 
Stellvertretend für die Gruppe der älteren Probanden ist in den folgenden Aktivierungskarten (Abbildung 37 und 38) ein 86-jähriger, männlicher Proband dargestellt. Eine PBR ist wie beim jungen Probanden im SI-Kortex auf der contralateralen Seite zu sehen, aber im Vergleich zum jungen Probanden ebenfalls auf der ipsilateralen Seite und damit eine stärkere Aktivierung.

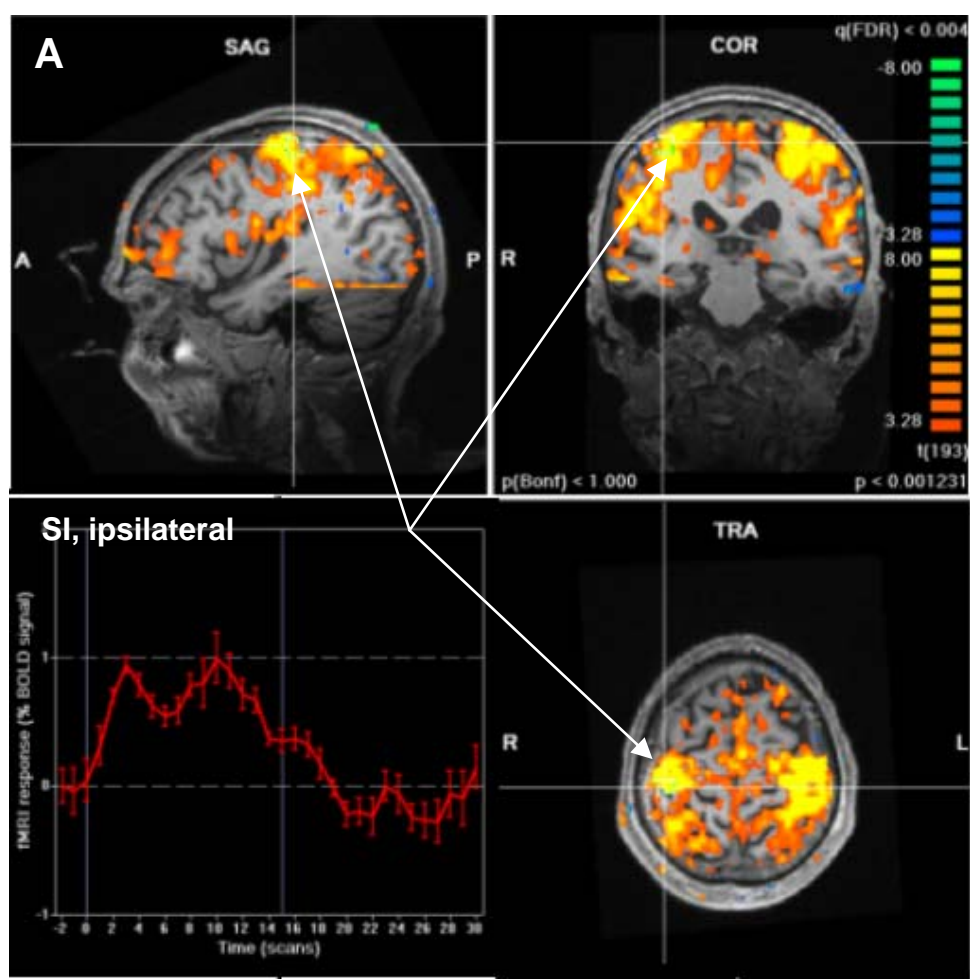

Abbildung 37: Alter Einzelproband mit Analyse der PBR im ipsilateralen SI-Kortex.

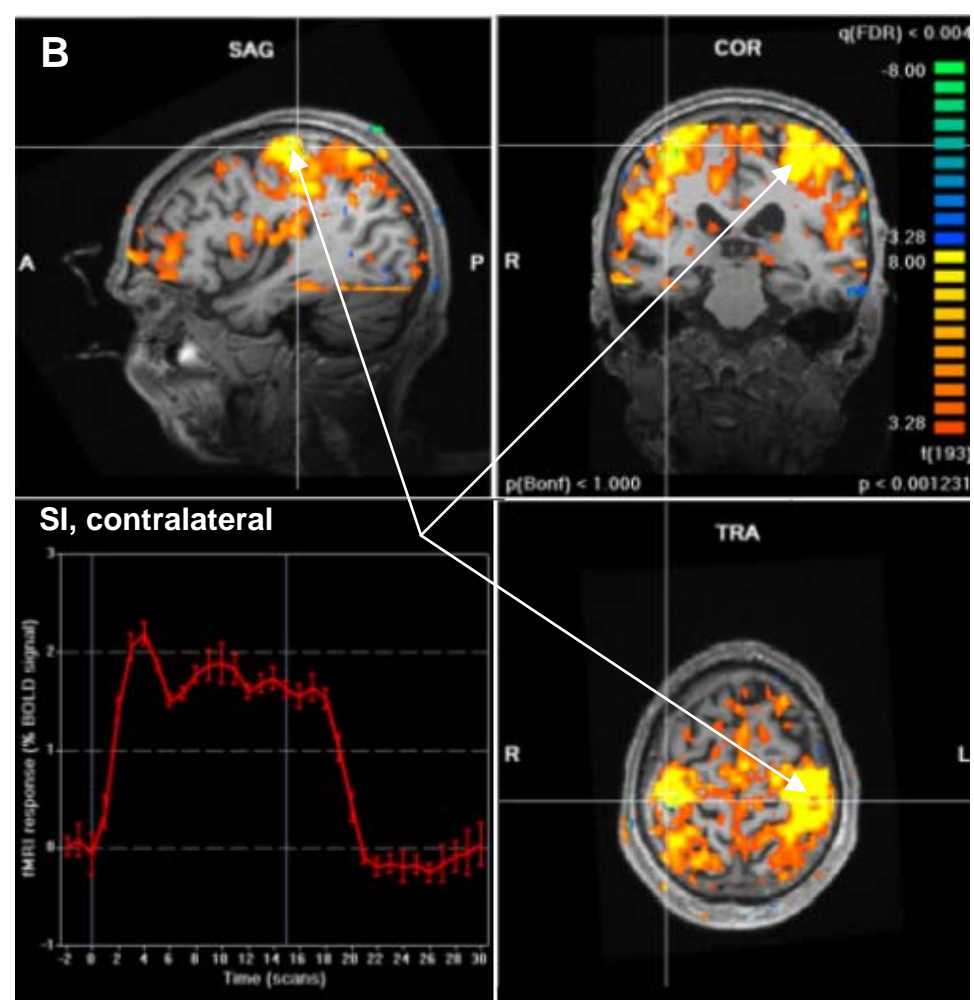

Abbildung 38: Alter Einzelproband mit Analyse der PBR im contralateralen SI-Kortex 


\subsubsection{Detaillierte Einzelbetrachtung der Probanden}

Bei der weiteren detaillierten Einzelbetrachtung (vorher Gruppen-Analysen) der von uns gemessenen jungen Probanden (Abbildung 39) fällt folgendes auf: Contralateral fallen die BOLD-Signal-Antworten durchweg positiv aus (100\%). Auf der ipsilateralen Seite sind die BOLD-Signal-Antworten bis auf zwei Probanden negativ (86\%). Bei diesen zwei Ausnahmen (P6 und P7) war keine markante BOLD-Signal-Änderung während der Stimulation zu sehen (Abbildung 39).

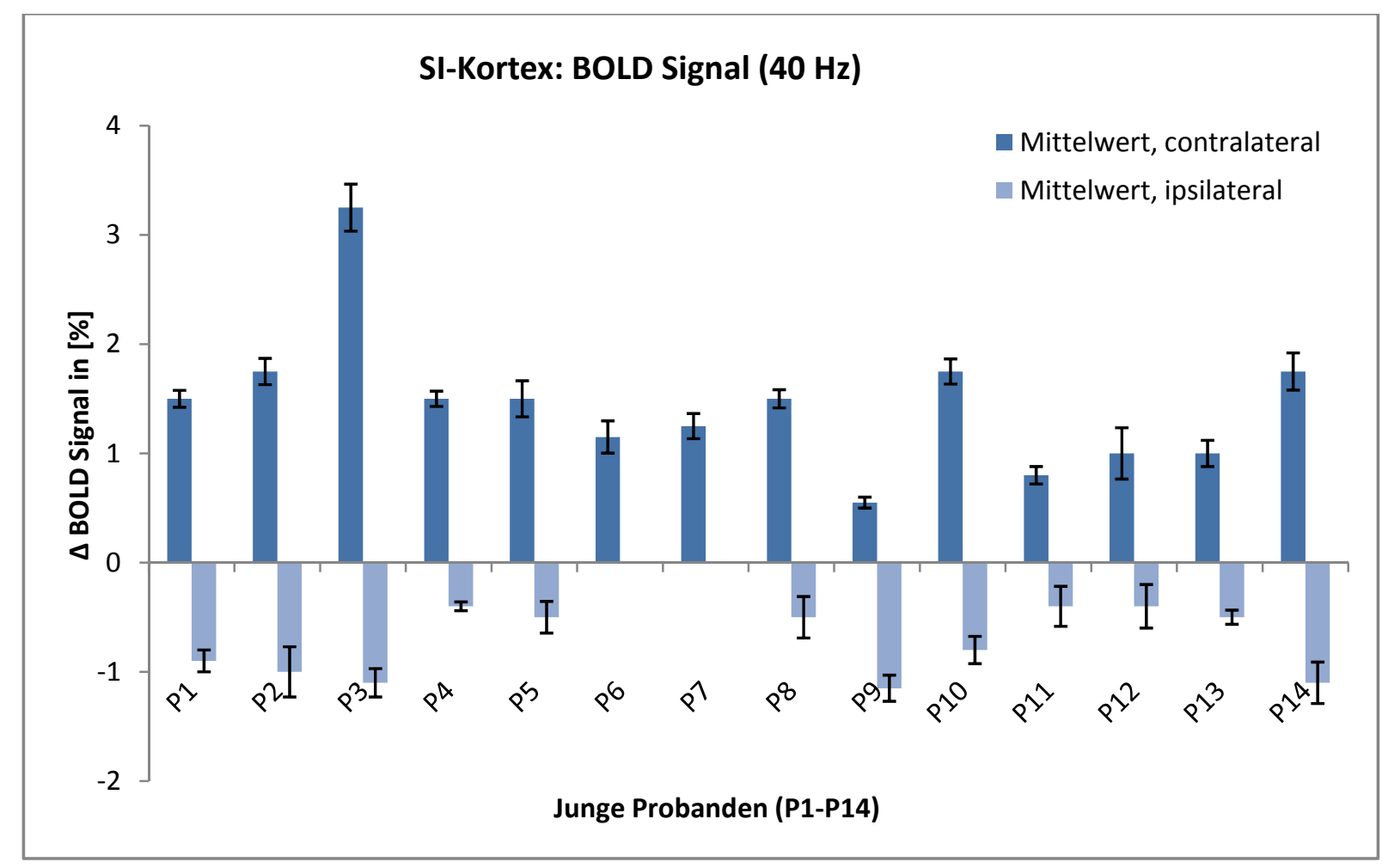

Abbildung 39: BOLD-Signal-Änderungen (Maximal- bzw. Minimalwerte) der 40-HzStimulation der jungen Probanden für den SI-Kortex bilateral, als Mittelwerte der 1. und 2. Runde der 40-Hz-Stimulation mit Standardabweichung (SD). 
Bei der Analyse der alten Einzelprobanden werden im Vergleich zu den jüngeren Probanden Unterschiede sichtbar. Contralateral fällt die BOLD-Signal-Antwort ebenfalls durchweg positiv aus (100\%). In zwei Fällen ist ipsilateral eine positive BOLD-Signal-Änderung zu erkennen (P3 und P7). Fünf Probanden zeigen eine NBR im ipsilateralen SI-Kortex (38\%), was in der Gruppenanalyse nicht so deutlich zu sehen war (Abbildung 40).

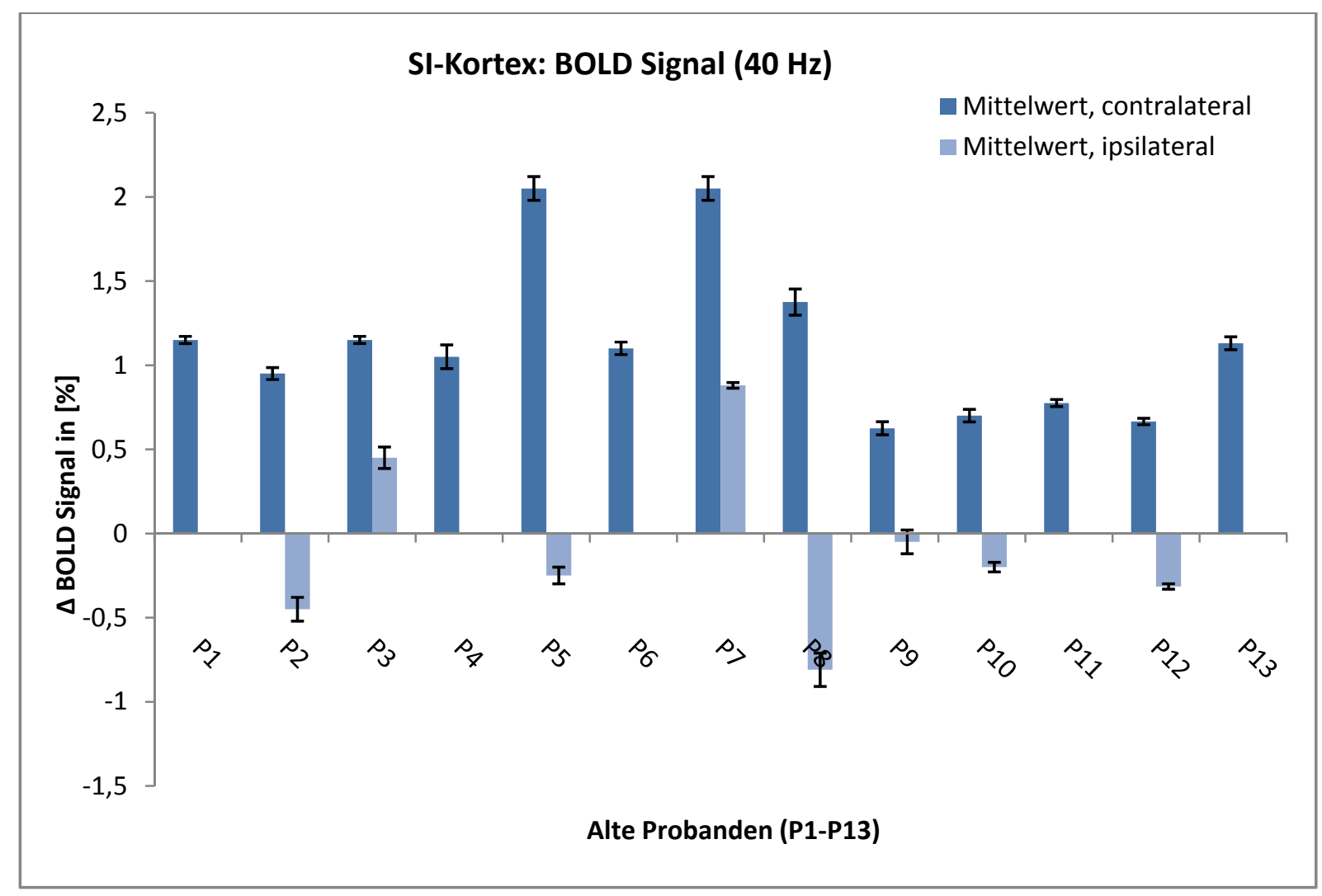

Abbildung 40: BOLD-Signal-Änderungen (Maximal- bzw. Minimalwerte) der 40-HzStimulation der alten Probanden im SI-Kortex bilateral, als Mittelwerte der 1. und 2. Runde der 40-Hz-Stimulation mit Standardabweichung (SD). 


\subsection{Ergebnisse der CPT-Messungen}

Die Analyse der psychophysiologischen Tests zeigt bei den jungen Probanden, dass die Mittelwerte der CPT-Messungen mit gleichzeitiger 40-Hz-Stimulation des rechten N. medianus durchweg über den Mittelwerten der Messungen während der Stimulations-Pausen (100\%) liegen (Abbildung 41).

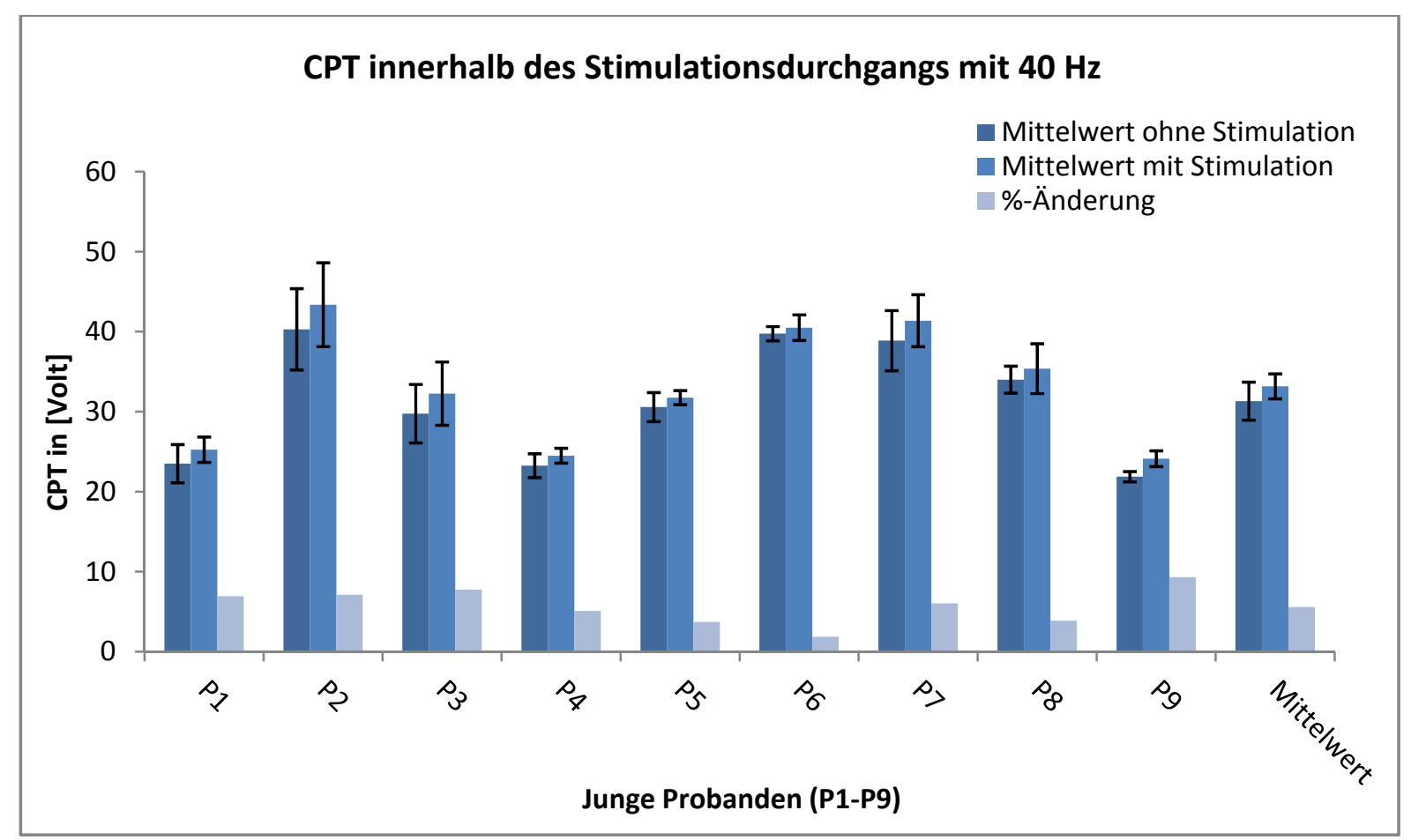

Abbildung 41: CPT-Veränderungen der jungen Probanden innerhalb des 40-HzStimulations-Durchgangs mit Standardabweichung (SD).

Für die gesamte junge Gruppe stiegen die CPT-Werte signifikant an $(p<0.001)$, um 6 $\pm 3 \%$ (Spannweite: 2-10\%). Das Beispiel eines jungen Einzelprobanden zeigt die Veränderung der CPT-Werte. Im fMRT zeigte dieser Proband im SI-Kortex ipsilateral eine NBR sowie contralateral eine PBR. Der Mittelwert im Stimulationsdurchgang mit Stimulus liegt über dem Mittelwert im Stimulationsdurchgang mit Stimulus-Pause (Tabelle 03). 
Tabelle 03: Absoluten Messwerte eines 23-jährigen, männlichen Probanden mit einer 40-HzStimulation bei 30 Volt. Die Messung erfolgte je 30 Sekunden mit und 30 Sekunden ohne Stimulation mit $40 \mathrm{~Hz}$.

\begin{tabular}{|c|c|c|}
\hline Zyklus & Messung & $\begin{array}{c}\text { Wert } \\
\text { [Volt] }\end{array}$ \\
\hline \hline 1 & ohne Stimulus & 34 \\
\hline 1 & mit Stimulus (40 Hz) & 37 \\
\hline 2 & ohne Stimulus & 37 \\
\hline 2 & mit Stimulus (40 Hz) & 40 \\
\hline 3 & ohne Stimulus & 35 \\
\hline 3 & mit Stimulus (40 Hz) & 39 \\
\hline 4 & ohne Stimulus & 36 \\
\hline 4 & mit Stimulus (40 Hz) & 38 \\
\hline 5 & ohne Stimulus & 42 \\
\hline 5 & mit Stimulus (40 Hz) & 44 \\
\hline 6 & ohne Stimulus & 43 \\
\hline 6 & mit Stimulus (40 Hz) & 45 \\
\hline 7 & ohne Stimulus & 43 \\
\hline 7 & mit Stimulus (40 Hz) & 43 \\
\hline 8 & ohne Stimulus & 41 \\
\hline 8 & mit Stimulus (40 Hz) & 45 \\
\hline
\end{tabular}

\begin{tabular}{|l|l|}
\hline Mittelwert Gesamt & $\mathbf{4 0 , 1 3} \pm 3, \mathbf{6 3}$ \\
\hline Mittelwert ohne Stimulus & $\mathbf{3 8 , 8 8} \pm 3,76$ \\
\hline Mittelwert mit Stimulus & $\mathbf{4 1 , 3 8 \pm 3 , 2 5}$ \\
\hline \hline \%-Änderung & $\mathbf{6 , 0 4}$ \\
\hline
\end{tabular}

Die Ergebnisse der psychophysiologischen Tests der alten Probanden zeigen, dass die Mittelwerte der Messungen mit gleichzeitiger 40-Hz-Stimulation des rechten $\mathrm{N}$. medianus leicht über den Mittelwerten der Messungen während der StimulationsPausen (100\%) liegen (Abbildung 42). Allerdings sind die \%-Änderungen wesentlich geringer als die der jüngeren Probanden (vergleiche Abbildung 41). 


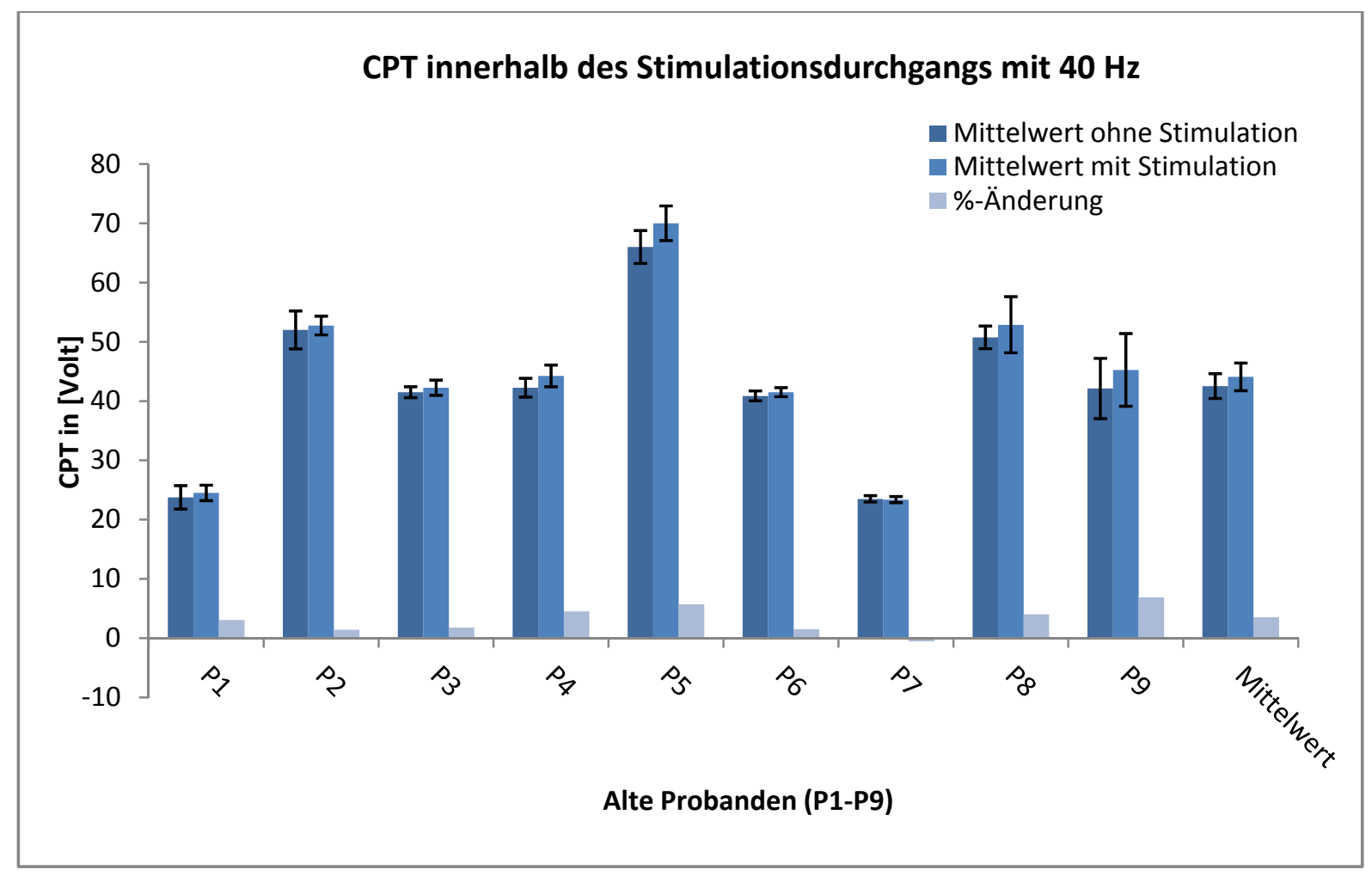

Abbildung 42: CPT-Veränderungen der alten Probanden innerhalb des 40-Hz-StimulationsDurchgangs mit Standardabweichung (SD).

Als Anmerkung sei zu erwähnen, dass die Nummerierungen der Probanden in den Abbildungen 41 und 42 (P1-P9) nicht mit den Nummerierungen der Probanden in den Abbildungen 39 (P1-14) und 40 (P1-13) übereinstimmen. Bei den CPTMessungen wurden die Probanden blind ausgewählt und es wurden aus organisatorischen Gründen auch nicht alle, die bei den 40-Hz-Stimulationen gemessen wurden, getestet.

Das Beispiel einer alten Einzelprobandin (Tabelle 04) zeigt wie bei dem zuvor gezeigten jüngeren Probanden (Tabelle 03) die Veränderungen der CPT-Werte. Im fMRT zeigte die alte Probandin (Tabelle 04) im SI-Kortex ipsilateral keine NBR sowie contralateral eine PBR. Der Mittelwert im Stimulationsdurchgang mit Stimulus gleicht annähernd dem Mittelwert im Stimulationsdurchgang mit Stimulus-Pause (Tabelle 04). Bei dem zuvor gezeigten jüngeren Probanden lag im Gegensatz dazu der Mittelwert mit Stimulus deutlicher über dem Mittelwert ohne Stimulus (Tabelle 03). 
Tabelle 04: Absoluten Messwerte einer 79-jährigen, weiblichen Probandin mit einer 40-HzStimulation bei 30 Volt. Die Messung erfolgte je 30 Sekunden mit und 30 Sekunden ohne Stimulation mit $40 \mathrm{~Hz}$.

\begin{tabular}{|c|c|c|}
\hline Zyklus & Messung & $\begin{array}{c}\text { Wert } \\
\text { [Volt] }\end{array}$ \\
\hline \hline 1 & ohne Stimulus & 59 \\
\hline 1 & mit Stimulus $(40 \mathrm{~Hz})$ & 53 \\
\hline 2 & ohne Stimulus & 50 \\
\hline 2 & mit Stimulus $(40 \mathrm{~Hz})$ & 50 \\
\hline 3 & ohne Stimulus & 50 \\
\hline 3 & mit Stimulus $(40 \mathrm{~Hz})$ & 53 \\
\hline 4 & ohne Stimulus & 50 \\
\hline 4 & mit Stimulus $(40 \mathrm{~Hz})$ & 55 \\
\hline 5 & ohne Stimulus & 49 \\
\hline 5 & mit Stimulus $(40 \mathrm{~Hz})$ & 53 \\
\hline 6 & ohne Stimulus & 53 \\
\hline 6 & mit Stimulus $(40 \mathrm{~Hz})$ & 54 \\
\hline 7 & ohne Stimulus & 53 \\
\hline 7 & mit Stimulus $(40 \mathrm{~Hz})$ & 53 \\
\hline 8 & ohne Stimulus & 52 \\
\hline 8 & mit Stimulus $(40 \mathrm{~Hz})$ & 51 \\
\hline
\end{tabular}

\begin{tabular}{|l|l|}
\hline Mittelwert Gesamt & $\mathbf{5 2} \pm 2,47$ \\
\hline Mittelwert ohne Stimulus & $\mathbf{5 2 \pm 3 , 2 1}$ \\
\hline Mittelwert mit Stimulus & $\mathbf{5 2 , 7 5 \pm 1 , 5 8}$ \\
\hline \hline \%-Änderung & $\mathbf{1 , 4 2}$ \\
\hline
\end{tabular}

Vergleicht man die Messungen beider Altersgruppen miteinander wird deutlich, dass die CPT beider Altersgruppen mit Stimulation ansteigt. Sie ist dementsprechend höher als in der Stimulations-Pause. Die Zunahme der \%-Änderung liegt bei den jüngeren Probanden allerdings über die der älteren (Abbildung 43). 


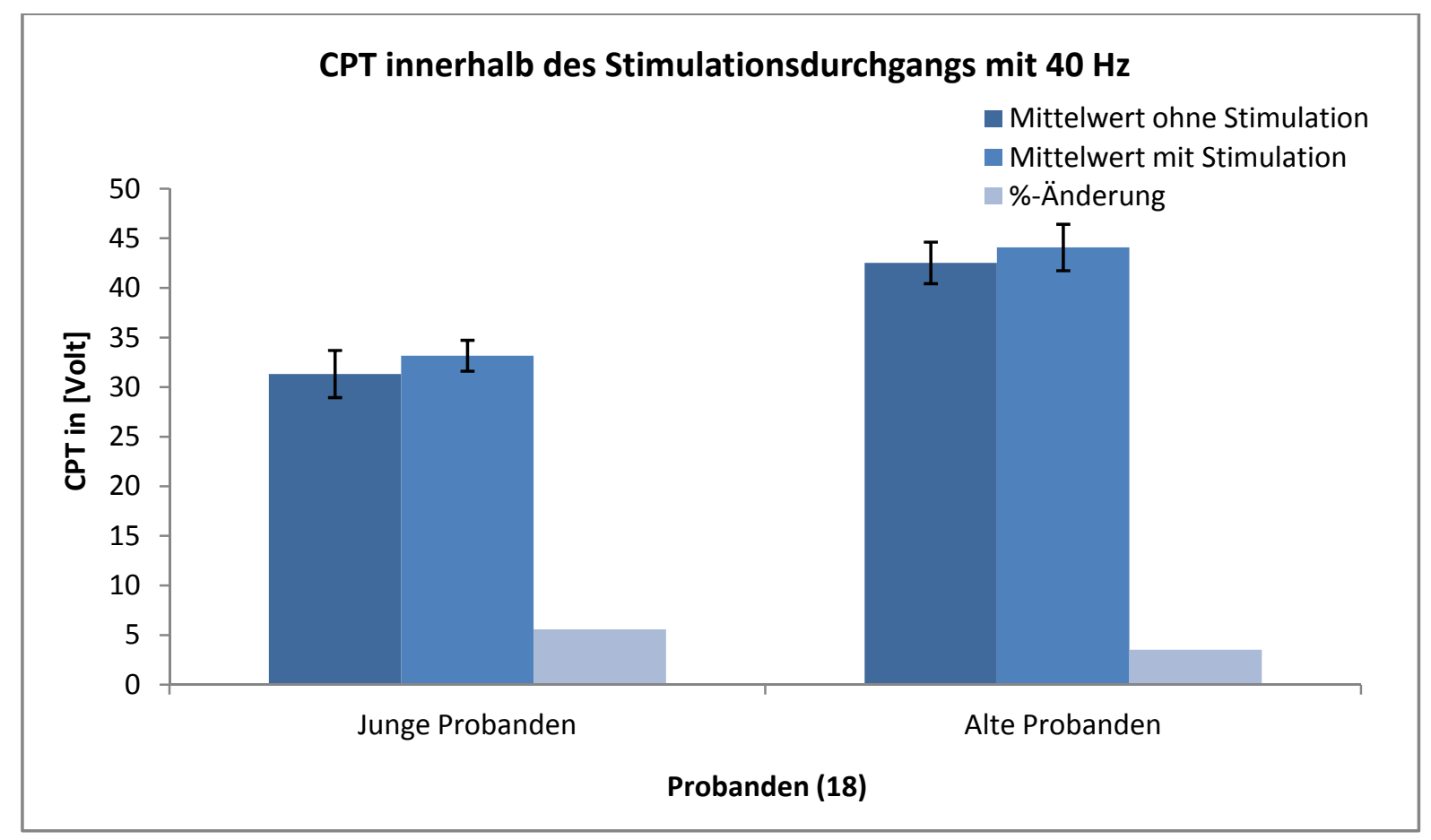

Abbildung 43: Mittelwerte der CPT-Änderungen der jungen und alten Gruppe innerhalb des Stimulationsdurchgangs mit Standardabweichung (SD).

Berechnet man den t-Test zwischen der jüngeren und der älteren Probandengruppe (\%-Änderung) fällt die Zunahme der CPT bei der jüngeren Gruppe wesentlich höher aus als bei den älteren. Contralateral und ipsilateral liegen die Werte der jüngeren Probanden über denen der älteren (Abbildung 44).

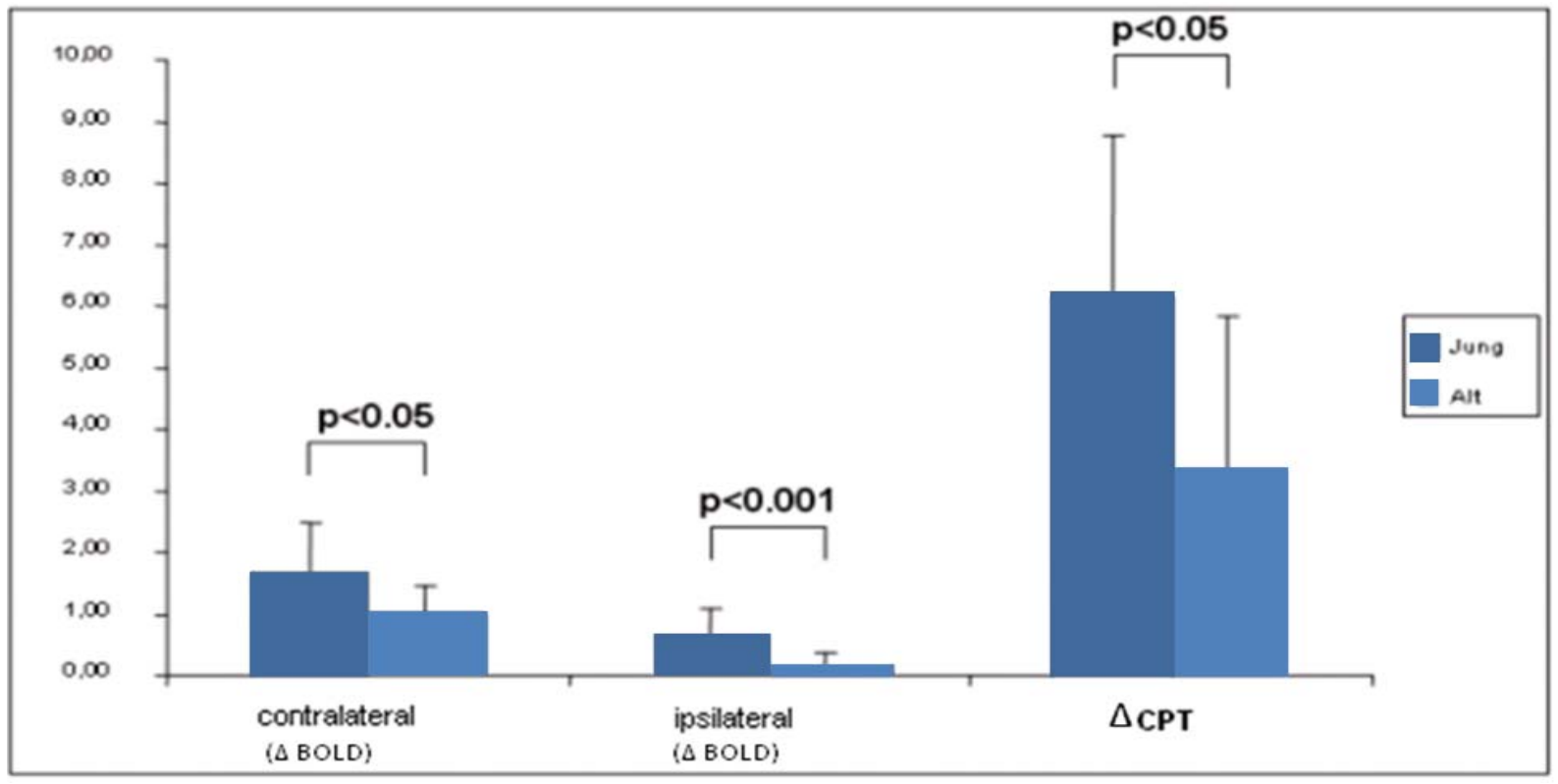

Abbildung 44: fMRT und psychophysiologische Experimente. T-Test zwischen \%-Änderung der jungen und alten Probanden. 


\section{4.) Diskussion}

In der vorliegenden Studie untersuchten wir mittels FMRT und gleichzeitiger elektrischer Stimulation des rechten N. medianus die funktionellen Zusammenhänge kortikaler Aktivierungen und Deaktivierungen und zwar insbesondere der NBR im ipsilateralen SI-Kortex während unilateraler sensorischer Stimulation. Den potenziellen Einfluss des Alters stellten wir mit dem Vergleich von zwei verschiedenen Altersgruppen dar. Im primären somatosensorischen Kortex fanden wir signifikante BOLD-Signal-Abfälle ipsilateral zur Stimulation des N. medianus hauptsächlich bei der jüngeren Gruppe (86\%) im Vergleich zur älteren (38\%) (Abbildung 24). Zusätzliche psychophysiologische CPT-Messungen zeigten, dass die fokalen, kortikalen NBR eine funktionelle und effektive Inhibition im somatosensorischen System der jüngeren Probanden widerspiegeln (Abbildung 44). Die ipsilateralen NBR korrelierten mit einem CPT-Anstieg auf der nicht stimulierten Seite bei elektrischer Wahrnehmungs-Schwellen-Reizung am contralateralen Zeigefinger. Bei den älteren Probanden konnte im Vergleich zu den jüngeren diese Korrelation nicht deutlich festgestellt werden. Die in diesem Experiment durchgeführte elektrische Stimulation des rechten N. medianus erzeugte in beiden Altersgruppen ein stabiles Abbild von positiver Aktivierung im hauptsächlich contralateralen, primären SI (100\%) (Abbildung 24) und bilateralen SII (100\%) des somatosensorischen Kortex (Abbildung 27). Diese Aktivierungs-Verhalten gleichen sich mit kürzlich absolvierten fMRT-, PET- und MEG-Studien, die zu ähnlichen Ergebnissen führten (Kampe et al. 2000, Nihashi et al. 2005).

Neben dem contralateralen SI-Kortexareal in dem die Aktivierungen stets am deutlichsten waren und damit mit einem höheren und stärkeren Maximum in beiden Altersgruppen auftraten (Abbildung 23), gab es weitere Kortexareale die Aktivierungen erzeugten. Diese waren jedoch in beiden Altersgruppen schwächer als die Amplituden der BOLD-Signal-Änderungen im contralateralen SI-Kortex (Abbildungen 30 bis 32 und Tabellen 01 und 02). Weitere aktuelle Messergebnisse zeigten ebenfalls bei vergangenen $\mathrm{N}$. medianus-Stimulationen die stärkste Aktivierung im contralateralen SI-Kortex (Nihashi et al. 2005). Ipsilateral waren die Amplituden der BOLD-Signal-Änderungen im SI-Kortex wesentlich schwächer und 
nicht bei allen Probanden zu sehen. Bei Nihashi et al. (2005) zeigten nur 6 von 10 Probanden ipsilaterale Aktivierungen. Diese Aktivierungen waren im Vergleich zu den contralateralen Aktivierungen im SI-Kortex vor allem weiter posterior und damit an einer anderen Lokalisation zu sehen. Bei ihren Messungen waren die 10 Probanden allerdings etwas älter als unsere jüngeren Probanden und jünger als unsere älteren Probanden. Somit unterscheiden sich unsere Messungen von Nihashi et al. (2005) etwas in der Probandenauswahl. Der SII-Kortex wurde bei Versuchen mit der N. medianus-Stimulation rechts und links abwechselnd bilateral aktiviert. Auf beiden Seiten kam man zum selben Ergebnis. Ein Teil der Probanden zeigte ebenfalls Aktivitäten im contralateralen Motor-Areal. Kakigi (1994) kam unseren Ergebnissen recht nahe, indem Aktivierungen im contralateralen SI, bilateral im SII und keine Aktivitäts-Änderungen im ipsilateralen SI festgestellt wurden. Daraus entstand der Vorschlag, dass der Weg der Aktivierung vom SI aus kommend bestimmten typischen Abläufen unterliegt. Der Ablauf der Aktivität im SI-Kortex ist heutzutage nicht vollkommen verstanden. Dennoch spielen mehrere Faktoren bei der Regulation der Aktivierung eine Rolle. Dazu zählen (1) die Miteinbeziehung des Corpus callosum vom contralateralen SI aus, (2) die direkte Projektion von ungekreuzten aufsteigenden Fasern zum ipsilateralen SI und Projektionen in beide, contralateralen und ipsilateralen SI vom Thalamus aus. Kanno et al. (2003) postulierten, dass ipsilaterale Aktivierungen des SI bei manchen Probanden als physiologisch normal vorkommen. Mit dem Unterschied, dass sie etwas weiter posterior als die contralateralen Antworten auftraten. Dies würde den Ergebnissen von Nihashi et al. (2005) entsprechen.

Zum Kurvenverlauf über die Zeit wurden bei Nihashi et al. (2005) sowie Logothetis et al. (2001) beobachtet, dass der Anstieg des BOLD-Signals mit einer leichten Latenz von 2-3 Sekunden nach Stimulationsbeginn stattfindet. Anschließend nach annähernd 7-10 Sekunden wird eine Plateauphase erreicht. Nach Beendigung des Stimulus kehrten die Aktivitäts-Amplituden zum Grund-Ausgangswert zurück. Unsere Zeitverlaufs-Ergebnisse der beiden Altersgruppen entsprechen etwa denen der vorhergegangenen Studien. Wie beobachtet, zeigten unsere Messungen der jungen und alten Probanden im Zeitverlauf ebenfalls Aktivitätsverzögerungen, Plateauphase und nach Beendigung die Rückkehr zum Ausgangsniveau (Abbildungen 25 und 26). Ähnliche Ergebnisse wie unsere wurden kürzlich während taktiler Stimulation von 
Hlushchuk und Hari (2006) publiziert. Sehr markant waren die PBR im contralateralen SI-Kortex und Deaktivierung im Kontrast dazu im ipsilateralen SIKortex. Die PBR war meist ausgeprägter. Unsere Ergebnisse stimmen damit überein, dass die PBR in ihrer Länge in beiden Altersgruppen während des Stimulus länger anhielten als die ipsilateralen NBR und einen stärkeren Maximalwert aufwiesen (Abbildungen 25 und 26). Dies ließe sich mit dem stärkeren Abfall von inhibitorischen Post-Synaptischen-Potenzialen (PSP) erklären (Hlushchuk und Hari 2006). Unsere beobachteten BOLD-Signal-Änderungen zeigten in beiden Altersgruppen charakteristisch unterschiedliche Zeitverläufe. Die PBR verliefen bei beiden tonisch und hielten fast über den gesamten Stimulationszeitraum an. Die NBR hingegen verliefen bei beiden Gruppen eher phasisch und nur kurzzeitig ins Negative. Sie kehrten noch während der Stimulationszeit-Periode zum Ausgangswert und Grundlinienniveau zurück. Die Amplitude der PBR hatte in den Messungen von Hlushchuk und Hari (2006) ebenso zumeist ein stärkeres Maximum im Vergleich zum stärksten Minimum der NBR. Die Extremwerte waren bei der PBR dementsprechend stärker. Die gelegentliche Deaktivierung der ipsilateralen SI-MI-Region wurde bereits in anderen Studien gesehen. Transkallosale und kortiko-kortikale Einflüsse spielen in der Literatur bei Aktivierungen und Deaktivierungen eine Rolle. Dabei stimulieren aktivierte sensomotorische Areale der einen Seite die Inhibition von sensomotorischen Arealen der Gegenseite. Diese Theorie wurde von vergangenen Studien unterstützt (Stefanovic et al. 2004, Newton et al. 2005, Hlushchuk und Hari 2006). NBR und neuronale Inhibition stehen folgendermaßen im Zusammenhang: Das BOLD-Signal korreliert mit neuronalen post-synaptischen Aktivitäten und lokalen Potenzialen und reflektiert eher den Input als den Output zu relevanten kortikalen Arealen (Logothetis und Wandell 2004). Ein Abfall des Inputs und damit der neuronalen Aktivität zeigt dementsprechend eine Deaktivierung im fMRT. Shmuel et al. (2006) zeigten mit intrakortikalen Messungen und gleichzeitigen fMRT-Aufnahmen einen engen Zusammenhang zwischen NBR und abfallender Aktivität von neuronaler Aktivität im primären, visuellen Kortex von Affen. Der Abfall der neuronalen Aktivität wird laut innen durch eine präsynaptische Inhibition ausgelöst. Der phasische Zeitverlauf der NBR, welcher in unserer Studie festgestellt wurde (Abbildung 25), ist in Übereinstimmung damit, dass inhibitorische postsynaptische Potenziale (IPSP) schneller abmildern (Deisz und Prince 1989). Prominente PBR waren wie bei unseren Messungen auch bei Grefkes et al. (2001) sowie Hlushchuk und Hari (2006) 
im contralateralen SI-Kortex und im bilateralen SII-Kortex zu sehen (Abbildungen 25 und 27). Statistische signifikante Deaktivierungen und damit NBR traten im ipsilateralen, primären sensomotorischen Kortex auf. Leichte Deaktivierungen fanden diese Gruppen noch links parieto-okzipital und rechts posterior-parietal sowie im contralateralen Cerebellum. Auch bei unseren Messungen zeigte sich eine NBR im contralateralen Cerebellum, allerdings nur bei den jüngeren Probanden. Bei den älteren Probanden zeigte sich keine BOLD-Signal-Änderung (Tabellen 01 und 02). Ipsilateral zeigten unsere jüngeren und älteren Probanden eine PBR im Cerebellum, wobei die PBR der älteren das der jüngeren Probanden leicht überstieg (Abbildungen 28 bis 30). Eine alte Vergleichsgruppe gab es bei der Studie von Hlushchuk und Hari (2006) allerdings nicht, so dass sich ihre Ergebnisse nur mit unseren jungen Probanden gleichen. Transkallosale Inhibition wird bei Hlushchuk und Hari (2006) und in weiteren Studien als eine mögliche Ursache des Auftretens von NBR im ipsilateralen SI-Kortex genannt. Dies könnte auf Grund von intrakortikalen SI-MIVerbindungen zu erklären sein, die im höheren Lebensalter ihre Aktivität umstellen und weniger stark inhibieren könnten wie unsere CPT-Vergleichsmessungen andeuten (Abbildungen 43 und 44).

Vergleichbar mit den CPT-Anstiegen bei unseren jüngeren Probanden bei gleichzeitiger N. medianus-Stimulation (vergleiche Abbildung 41) wurde in einer Studie von Seyal et al. (1995) Folgendes gezeigt: Bei transkranieller, magnetischer Stimulation auf den ipsilateralen, parietalen Kortex kam es bei gleichzeitiger Stimulation der Hand auf der gleichen Seite zu einem Anstieg der contralateralen, taktilen Sensitivität. Sie definierten diesen Effekt als Unterbrechung der Inhibition. Bei unseren Versuchen stieg die CPT am contralateralen Zeigefinger vor allem bei den jüngeren Probanden deutlicher an als bei den älteren, die weniger NBR aufwiesen (Abbildung 43). Bei Patienten mit ipsilateraler NBR, wie vor allem bei den jüngeren Probanden gesehen, fand dementsprechend eine Inhibition statt (Abbildung 44). Diese Feststellungen wurden in ähnlicher Art und Weise bei invasiven Studien im Tiermodell bei Affen und Ratten dargestellt, bei denen Inhibition in der Verarbeitung von optischen Reizen eine Rolle spielte (Clarey et al. 1996, Pluto et al. 2005). Auf Grund dessen, dass beide Hemisphären des ZNS normalerweise von unterschiedlichen Gefäß-Gebieten versorgt werden, können die NBR, welche wir hauptsächlich bei den jüngeren Probanden gefunden haben, eher nicht durch einen 
kapillären Steal-Effekt zu Stande kommen. Eine andere Ursache ist vermutlich der Grund. Neuroinhibtion und -regulation spielen eine Kontrollfunktion. Die NBR-Werte des ipsilateralen SI-Kortex auf der einen Seite und der Anstieg der CPT am linken Zeigefinger während elektrischer Stimulation des rechten N. medianus, auf der anderen Seite, stehen im Zusammenhang (Abbildung 44). Zeigte sich eine besonders negative Amplitude der NBR, so stieg die CPT signifikant an. Die CPTÄnderungen dieser Werte korrelierten bei den jüngeren Probanden im Gegensatz zu den älteren sehr gut mit den negativen NBR, die wir in den fMRT-Messungen erhielten. Diese Feststellung unterstützt die These, dass NBR eine effektive funktionelle Inhibition der neuronal feuernden Strukturen darstellen beziehungsweise im Zusammenhang stehen. In einer anderen Studie konnten durch nicht wahrnehmbare elektrische Finger-Stimulation induzierte NBR ebenfalls mit ansteigenden CPT-Werten in Zusammenhang gebracht werden (Blankenburg et al. 2003). Interessant ist weiterhin die Beobachtung, dass sich die PBR nach der ersten Ausführung erlernter motorischer Bewegungs-Aufgaben bei wiederholt folgenden Durchführungen verringerten oder sich später nach ausgiebigem Training der Aufgabe sogar eine NBR zeigte (Hummel et al. 2004). Dass es einen engen Zusammenhang zwischen der NBR und dem Abfall von neuronaler Aktivität gibt, zeigten unter anderem Messungen im visuellen Kortex von anästhesierten Affen, bei denen es neben aktivierten Arealen ein paar Millimeter entfernt in frühen Arealen des visuellen Kortex (V1) deaktivierte Areale zu beobachten gab (Shmuel et al. 2006).

Es erscheint am wahrscheinlichsten, dass eine neuronal gesteuerte Inhibition die Veränderungen in CBF und der hämodynamischen Regulation beeinflusst (Arthurs et al. 2007). Dies kann auf Grund von neuronalen Steuerungen wie überwiegend bei unseren jüngeren Probanden in funktionell im Zusammenhang stehenden Arealen unilateral und auch bilateral geschehen. Ipsilaterale kortikale Antworten hängen enger mit den contralateralen Antworten zusammen als mit der Stimulus-Intensität an sich. Bei schlafenden Probanden wurde in EEG-Studien nach akustisch evozierten Potenzialen eine positive Korrelation zwischen deutlichem Abfall der Amplitude von NBR und der EEG-Synchronisation gezeigt. Dies zeigt einen Zusammenhang zwischen kortikaler Deaktivierung und NBR (Czisch et al. 2004). Da in verschiedenen Studien (Arthurs et al. 2007) die Amplitude der PBR auf der contralateralen Seite zur N. medianus-Stimulation und die negative Amplitude der NBR auf der ipsilateralen 
Seite korrelierten, müsste es möglich sein, dass beispielsweise bei Läsionen des Corpus callosum diese neuronalen Verbindungen unterbrochen werden könnten und somit dieses mögliche Steuerungssystem nicht mehr funktioniert (Bezug zu Abbildung 44). Damit könnte kein signifikanter Zusammenhang mehr sichtbar sein zwischen der Aktivität der Kortexareale. Bei Läsionen in diesem Bereich, wie beispielsweise nach Traumata, Schlaganfällen oder Altersdegeneration müssten fMRT-Messungen unter anderen Gesichtspunkten betrachtet werden als das normale, gesunde ZNS von Probanden. Simultane Stimulationen und Unterdrückungen von funktionell im Zusammenhang stehenden kortikalen Arealen über längere anatomische Distanzen, wie beispielsweise interhemisphäriell sowie die Miteinbeziehung von neuro-vaskulären Steuerungen, scheinen unter komplexen Steuerungsmechanismen und Verbindungen zu stehen (Arthurs et al. 2007). Der Aktivierungsweg des ipsilateralen SI-Kortex könnte folgendermaßen aussehen: Sutherland und Tang (2006) schlagen verschiedene Theorien für die Herkunft der De- und Aktivierung des ipsilateralen SI-Kortex vor. Es könnte (1) an einer Aktivierung durch Inputs des contralateralen SI zustande kommen, (2) durch ungekreuzte ipsilaterale Inputs oder (3) durch Inputs von höheren Kortexarealen. Transkallosale Inputs hängen laut Sutherland und Tang (2006) mit der Beschaffenheit des Corpus callosum und seiner Funktion als Struktur für kreuzende Bahnen zwischen den Hemisphären zusammen. Bei Primaten untersuchten diesen Zusammenhang Fabri et al. (2001) genauer indem sie das Corpus callosum resezierten. Dabei verschwanden wie oben vermutet die hämodynamischen Antworten im ipsilateralen SI-Kortex. Diese Feststellung hängt laut ihrer Untersuchungen auch mit dem Alter und damit dem Reifungsgrad und der Größe des Corpus callosum zusammen. Sichtbar war dies an der Amplitude und dem Ausmaß der ipsilateralen SI-Kortex-Antwort. Auch die Latenzzeit bis zur Aktivierung des ipsilateralen SI-Kortex stand in Zusammenhang mit der Corpus-callosum-Intaktheit. Diese betrug contralateral 20 Millisekunden und ipsilateral 45 Millisekunden. Kanno et al. (2003) wiesen darauf hin, dass es aber auch ungekreuzte Bahnen sein könnten, die den ipsilateralen SI-Kortex aktivieren. Sie zeigten in einer weiteren Studie mit zwei Patienten mit schweren Schäden in der linken Hemisphäre, dass diese im ipsilateralen SI-Kortex der nicht geschädigten rechten Seite deutliche Signale aufwiesen, während contralateral auf der geschädigten Seite keine AktivitätsSignale zu sehen waren. Dieses Beispiel zeigt, dass es hier unabhängig vom SI- 
Kortex auf der contralateralen Seite zu einem Aktivitäts-Anstieg auf der ipsilateralen Seite kam. Dies könnte auf ungekreuzte Fasern hinweisen. Die dritte Möglichkeit der Aktivierung des ipsilateralen SI-Kortex könnte durch höher liegende Zentren geschehen wie beispielsweise durch den SII-Kortex. Da aber der SII-Kortex meist später mit einer Latenz von 70 Millisekunden im Vergleich zum SI-Kortex mit 45 Millisekunden aktiviert wird, ist es unwahrscheinlich, dass dieser Aktivierungsweg maßgeblich eine Rolle zu spielen scheint (Sutherland und Tang 2006). Obwohl es anscheinend auch ungekreuzte Aktivierungswege des ipsilateralen SI-Kortex gibt, werden zusammenfassend in der Literatur als Hauptgrund die gekreuzten Aktivierungswege via Corpus callosum vom SI-Kortex der contralateralen Seite ausgehend angesehen, welche im höheren Lebensalter ihre Aktivierung umstellen und weniger zu inhibieren scheinen (Sutherland und Tang 2006).

Die methodischen Ungenauigkeiten unserer Messungen stellen sich folgendermaßen da. Die von uns angewendete Methode entspricht etwa dem Messaufbau von vorhergegangenen Studien (Hlushchuck und Hari 2006). Wir benutzten eine elektrische Stimulation des N. medianus zur Aktivierung des somatosensorischen Kortex. Diese Art von Stimulation wird zunehmend zum Charakterisieren des somatosensorischen Systems genutzt. Sie umgeht allerdings Mechanorezeptoren und produziert umfangreiche afferente Salven und unterscheidet sich somit von natürlichen taktilen Stimulationen, von zum Beispiel der Haut, im Alltag. Andererseits wurden kürzlich annäherungsweise identische NBR sowie die dazugehörigen Zeitverläufe während taktiler Stimulation beobachtet (Hlushchuck und Hari 2006). Dies deutet an, dass unsere Ergebnisse nicht nur einmalig in Bezug auf elektrische Stimulationen sind und die angewandte Methode durchaus sinnvoll ist. Unser Experiment-Aufbau erlaubte uns während sensorischer Stimulation leider nur einen einzelnen CPT-Wert beim direkten Vergleich zwischen CPT- und BOLD-SignalÄnderung zu messen. Wir haben mit unserer Methode eine ipsilaterale NBR im primären somatosensorischen Kortex während unilateraler elektrischer Stimulation des N. medianus bei jüngeren Probanden (83\%) dargestellt und nachgewiesen (Abbildung 26). Bei Älteren war dies geringer vorhanden (38\%). Basierend auf zusätzlichen psychophysiologischen CPT-Experimenten behaupten wir, dass diese negativen BOLD-Signal-Änderungen eine funktionelle und effektive Inhibition innerhalb des somatosensorischen Systems widerspiegeln, welche im höheren 
Lebensalter deutlich verringert auftritt. Wie erwartet konnte ein Einfluss der Messung der Wahrnehmungsschwelle auf das BOLD-Signal bei unseren Messungen nicht festgestellt werden. Des Weiteren ist anzumerken, dass wir lediglich drei Probanden bezüglich der ROI-Referenz und der zeitgleichen CPT-Messung untersucht haben. Bei den restlichen Probanden wurde angenommen, dass die ROI dem Repräsentationsgebiet des Handareals der drei untersuchten Probanden entspricht. Beim Vergleich der Stimulationsarten fielen Franceschini et al. (2003) Folgendes auf: Sie stellten bei unterschiedlichen Messungen der Kortex-Aktivität mit unilateralem FT, taktiler Stimulation und elektrischer Stimulation des N. medianus fest, dass es zu einem deutlichen Anstieg im sensomotorischen Kortex auf der contralateralen Seite zum Stimulus kam. Zum Vergleich nahmen sie Messungen an der rechten und linken Hand vor. Die stärkste Veränderung der Aktivierungsamplitude der KortexAktivierung stellten sie beim aktiven FT im Vergleich zu den anderen zwei Stimulationsmethoden fest. Die elektrische Stimulation des N. medianus zeigte hierbei das geringste Maximum im Vergleich zum FT oder der taktilen Stimulation. Die ipsilaterale Aktivität fiel geringer aus als die contralaterale. Unsere Messungen zeigten ebenfalls, dass es im Vergleich zu den elektrischen, passiven Stimulationen bei beiden Altersgruppen zu stärkeren BOLD-Signal-Änderungen beim FT kam (vergleiche Abbildungen 18 und 23). Als ein Nachteil der FT-Messungen wird die Durchführungs- und Leistungsabhängigkeit angesehen. Letztendlich wollten wir beim FT aber hauptsächlich zeigen, wie die allgemeine hämodynamische Antwort bei den älteren Probanden im Vergleich zu den jüngeren aussieht.

Unsere älteren Probanden zeigten im Vergleich zu den jüngeren beim FT eine stärkere Aktivierung in drei Qualitäten: (1) Maximum der Aktivierung (Abbildungen 18 und 19), (2) Steigung des Signals und (3) Anzahl der aktivierten Kortexareale (Abbildungen 20 und 21). Dieses Ergebnis unterstützt in ähnlicher Art und Weise die Aussagen anderer Studien (Mattay et al. 2002, Ward und Frackowiak 2003). Grundsätzlich lässt sich folglich sagen, dass in unseren sowie in anderen Studien beim FT festgestellt werden konnte, dass sich die altersbezogenen Aktivierungen während motorischer Aufgaben in diversen Qualitäten unterscheiden. Dazu zählen (1) Unterschiede in der hämodynamischen Antwort, (2) vermehrte kompensatorische Rekrutierung von Kortexarealen bei den alten Probanden, um gleiche Aufgaben ausführen zu können wie die jüngeren und (3) Kortex-Aktivitäten ohne direkten Effekt 
auf die Durchführung von Aufgaben (Riecker et al. 2006). Die älteren Probanden brauchten folglich mehr Kortexareale mit höherem Aktivitätsmaximum, um im Vergleich zur jüngeren Gruppe die gleiche Antwort und damit Reaktion auf einen gleichwertigen Reiz zu erreichen (vergleiche Abbildungen 20 und 21). Die jüngeren Probanden reagierten ökonomisierter mit weniger Aktivierung und demselben Effekt. Man könnte es auch als eine Art physiologischen Verlust oder Verminderung der Reizbarkeit von interhemisphärischen oder intrakortikalen, inhibitorischen Aktivierungsverläufen mit zunehmendem Alter nennen (Riecker et al. 2006). Es stellt sich die Frage wie progredient dieser Verlauf ist und ab welchem Alter mit einer besonders signifikanten Veränderung zu rechnen wäre? Wann ist eine deutliche für einen Menschen und seine Umgebung spürbare, kognitive Veränderung bemerkbar? Übereinstimmend sind unsere FT-Ergebnisse mit Studien, die herausfanden, dass es altersbezogene Überaktivierungen im Motorkortex (Abbildungen 20 und 21) bei ausgeführten Bewegungen der Hand gibt (Riecker et al. 2006). Bei den FTMessungen sowie bei der Messung der CPT unter der 40-Hz-Stimulation wurde folglich deutlich, dass sich ältere im Vergleich zu unseren jüngeren Probanden schwieriger anpassten. Ältere Probanden passen sich im Vergleich zu jüngeren in weniger konzentrierten und weniger kleinen, fokal lokalisierten Kortexarealen auf verschiedene motorische oder kognitive Aufgaben sowie WahrnehmungsÄnderungen an. PET-Studien zeigten bei Gedächtnis-Tests und dem Erinnern von Gesichtern einen Abfall von Aktivität, der nicht anders zu kompensieren war bei älteren Probanden (Madden et al. 1999). Die älteren Probanden hatten folglich Schwierigkeiten beim Wiedererkennen. In anderen PET-Studien wurde beim Wiederholen von gesprochenen Sätzen und Wörtern gezeigt, dass ältere Probanden zur Kompensation um das gleiche Outcome zu erhalten wie jüngere, zusätzliche Kortexareale aktivierten (Cabeza et al. 1997). Die älteren Probanden reagierten langsamer und nicht in der gleichen Qualität wie die jüngeren. Sie zeigten bei Sailer et al. (2000) sowie in unserer Studie vermehrte bilaterale Aktivitäten im Vergleich zu den jüngeren Probanden bei motorischer Stimulation (Abbildung 20 und 21). Probanden, die in einem schlechteren psychologischen, psychiatrischen und physischen Allgemeinzustand waren, zeigten eine Verschiebung hin zu anderen Kortexarealen und alternativen kortikalen Systemen im Vergleich zu älteren Probanden, die sich geistig in einem sehr guten Allgemeinzustand befanden. Das Hinzuziehen von zusätzlichen kortikalen Arealen ist bei komplexeren und 
anspruchsvolleren Aufgaben oft nicht ausreichend genug, um das gleiche Ergebnis wie bei jüngeren Probanden zu erzielen. Ist die Aufgabe im Schwierigkeitsgrad zu anspruchsvoll, so ist die Durchführung nicht gleich gut genug im Vergleich zu den jüngeren Probanden. Die altersbezogene Miteinbeziehung zusätzlicher kortikaler Areale wurde in der Vergangenheit oft als eine Art kompensatorischer Mechanismus angesehen, der primäre Areale, die ungenügend aktiviert werden können, unterstützt (Cabeza et al. 1997). Das kann folglich ein gezielter Mechanismus sein, um mit einem positiven Effekt auf die Umsetzung von motorischen Aufgaben einzuwirken oder aber eine ungenügende Strategie, weil inhibitorische Prozesse degenerieren. Die Feststellung, dass die Hinzunahme zusätzlicher Areale bei motorischen Aufgaben im Gegensatz zu Probanden in einem schlechten Zustand vor allem bei Probanden im guten geistigen und körperlichen Allgemeinzustand funktioniert, weist darauf hin, dass es sich nicht unbedingt um eine ineffektive Strategie des alternden ZNS handelt (Sailer et al. 2000). Ältere Probanden, die wie in unseren Messungen zusätzliche Kortexareale aktivieren, müssten dementsprechend Probanden in einem gutem Allgemeinzustand sein (Sailer et al. 2000).

Cabeza (2002) beschreibt in seinen Untersuchungen das HAROLD-Model (HAROLD: Hypothesis of $\underline{H}$ emispheric Asymmetry Reduction on Older Adults). Damit wird der Verlust der Asymmetrie von Aktivierungen im Kortex von älteren Probanden beschrieben. Bei jüngeren Probanden wurde unter gleichen Bedingungen wie bei älteren das Vorhandensein einer stärkeren Asymmetrie beschrieben. Die jüngeren Probanden aktivierten unter bestimmten Aufgaben nicht bilateral ähnliche Kortexareale, sondern unilateral auf eine Hemisphäre beschränkt (siehe contralateraler SI-Kortex). Diese Theorie passt unter anderen zu den Ergebnissen unserer Messungen des SI-Kortex bei passiver N. medianus-Stimulation (Abbildung 24) oder des FT (Abbildungen 20 und 21). Das HAROLD-Modell beschreibt, dass es mit höherem Lebensalter zu vermehrt bilateralen Aktivitäten auf bestimmte Stimuli kommt. Altersbezogene hemisphärische Asymmetrie-Reduktion kann laut HAROLDHypothese ein kompensatorischer Mechanismus sein oder ein UmdifferenzierungsProzess. Die Theorie, dass es im älteren ZNS zu vermehrter Aktivierung von Kortexarealen bei verschiedenen Stimuli und damit einer erhöhten Verbindung und verringerten Inhibition kommt, muss nicht unbedingt als ein Nachteil gewertet werden. Es ist eine Art Hilfsprozess, um kompensatorisch Aushilfe zu verschaffen, 
weil bestimmte neuronale Strukturen nicht mehr adäquat funktionieren (Rowe et al. 2006).

Die Kombination aus dem Verlust von weißer Substanz, Demyelinisierung und Zelluntergang im Ziel-Kortex reduzieren die Effektivität von distanzierten kortikokortikalen Verbindungen von der prämotorischen Seite der Stimulation zu entfernten Arealen (Rowe et al. 2006). Transkallosale Effekte und Verbindungen verändern sich dementsprechend im höheren Lebensalter. Es kommt vermehrt zum Anstieg bilateraler Aktivität und zur Rekrutierung von mehr Kortexarealen im beispielsweise prämotorischen Kortex (vergleiche Abbildungen 20, 21 und 24). Komplexe Faktoren spielen für den Abfall der Motoraktivität im höheren Lebensalter eine Rolle. Dazu zählen Veränderungen in der muskuloskeletalen Architektur (Lexell 1997, Dutta et al. 1997), der peripheren und zentrale Nerven-Leitungen (Dorfman und Bosley 1979), Propriozeption (Kaplan et al. 1985) und neuromuskuläre Verbindungen (Delbono 2003). Es wird oft angenommen, dass es beim normalen Altern im höheren Lebensalter zu einem progressiven Verlust von kortikalen und striatalen Neuronen kommt. Hinweise sprechen jedoch gegen diese Annahme. So gibt es beispielsweise keinen signifikanten Verlust von Neuronen im primären Motorkortex (Haug und Eggers 1991). Allerdings gibt es Hinweise auf die Unterbrechungen der Intaktheit der weißen Substanz und damit der kortiko-kortikalen und kortiko-spinalen Verbindungen (Madden et al. 2004). Zu sehen waren diese Effekte von einfachen motorischen Aufgaben mit wiederholtem FT (Shimoyama et al. 1990), bis hin zu anspruchsvolleren Aufgaben, visuell gesteuerten Tests (Smith CD et al. 1999) sowie somatosensorischen und visuell gesteuerten Kontrollen von Fertigkeiten (Cole et al. 1999). Interessanterweise zeigten Schizophrenie-Patienten mit der Fehlfunktion von interkortikalen Verbindungen (Weinberger 1995, Mattay et al. 1997) ebenfalls bilaterale Aktivitäten des sensomotorischen Kortex wie unsere älteren Probanden und weniger transkallosale Inhibition.

Kommt es zu einer Veränderung der neurovaskulären Kopplung bei beispielsweise arteriellen Stenosen, so ändert sich das Ausmaß der hämodynamischen Antwort. Bei älteren Probanden kann es aufgrund von potenziell unterschiedlichen Gefäßdegenerationsprozessen daraufhin zu variierenden neurovaskulären Schaltungen und damit hämodynamischen Antworten kommen. Rother et al. (2002) 
beschrieben einen Patient der eine verstärkte Schwäche im rechten Arm hatte. Bei genauerer Untersuchung fand man Stenosen beider Carotiden und einer VertebralArterie. Der Abfall der neuralen Aktivität war relativ normal. Aber es kam zu keinem normalen größeren Anstieg des BOLD-Signals, eher zu einer verstärkten relativen desoxy-Hb-Konzentration. Der CBF und das mitfolgende oxy-Hb konnten nicht ausreichend bereitgestellt werden auf Grund der Gefäßstenosen. Bei neurovaskulärer Entkopplung kann es also zu einer Reduktion der Beurteilung und Abschätzung der Signalveränderugen bei bestimmten motorischen Aufgaben kommen. Bei älteren Probanden ist folglich eine realistische Interpretation potenziell schwieriger zu gewährleisten.

Verschiedene Studien befassten sich mit dem Einfluss des Alterns auf den sensomotorischen Kortex. Doch unterschieden sie sich in ihrem Untersuchungsschwerpunkt. Handelt es sich um reduzierte neuronale Aktivität oder in der Tat um eine ineffektive vaskuläre Reaktion bei gleichwertig funktionierenden neuronalen Aktivitäten bei jüngeren und älteren Probanden? Manche Studien berichteten nur über bestimmte Abgrenzungen im Kortex (D’Esposito et al. 1999), andere wiederum konzentrierten sich auf das Ausmaß der Aktivität (Tekes et al. 2005). Weitere legten ihr Hauptaugenmerk auf den primären und sekundären Motorkortex und Aktivitäten bei einfachen Aufgaben. Als einen wichtigen Grund für die von uns gemessenen Unterschiede zwischen jüngeren und älteren Probanden stehen die Feststellungen von O'Sullivan et al. (2001). Sie fanden heraus, dass Altern mit Veränderungen der weißen Substanz zusammenhängt. Es fanden sich mit Diffusions-Spannungs-Bildgebung (DTI: Diffusion Tensor İmaging) Hinweise für eine verminderte Intaktheit der Bahnen und angestiegene Wasser-Diffusion der weißen Substanz von älteren Probanden. Die Autoren schlagen vor, dass sich diese altersbezogenen Veränderungen der Intaktheit der weißen Substanz auf einen Abfall der funktionellen Intaktheit zwischen kortikalen ZNS-Regionen beziehen. Damit wird wie bereits vermutet ein kortikaler Verbindungsabbau beschrieben und folglich auch ein Verlust von mögichen Inhibitionsmechanismen. Das physiologische Ergebnis dieser Veränderungen der weißen Substanz, kann in höherem Lebensalter das nichtselektive Rekrutieren von Verarbeitungs-Regionen bedeuten wie es beispielsweise bei Logan et al. (2002) oder unseren Messungen gezeigt wurde. So kann es möglicherweise zu Kontrollverlusten oder Bewegungs-Einbußen kommen, wie es bei 
motorischen Aufgaben im höheren Lebensalter oft beobachtet wird (Logan et al. 2002).

\section{5.) Zusammenfassung}

Die funktionelle Magnetresonanz-Tomographie, basierend auf dem BOLD-Effekt, beinhaltet typischerweise die Suche nach positiver Kortex-Aktivierung während einer bestimmten Aufgabe zu einem kontrollierten Zustand. Die taktile Stimulation via peripherer elektrischer Nerven-Stimulation ergibt in fMRT-Studien eine messbare Aktivierung des somatosensiblen Systems. Neben den positiven BOLD-SignalÄnderungen im contralateralen, primären Kortex (Abbildung 25) gibt es jedoch zunehmend Hinweise, dass negative BOLD-Antworten während dieser Stimulation ebenfalls auftreten können (Abbildung 26). Obwohl in der Literatur allgemein die Meinung vertreten wird, dass diese neuronalen BOLD-Signal-Änderungen eine Unterdrückung neuronaler Aktivität darstellen, sind direkte Nachweise für diese These in unterschiedlichen Altersgruppen bislang selten. Für das somatosensible System gibt es seit längerer Zeit die Möglichkeit der Durchführung von quantitativen Messungen der sensorischen Funktion während gleichzeitiger Aktivierung und Stimulation. In der Vergangenheit wurde dieses System mit der fMRT-Technik sehr gut charakterisiert und dargestellt.

Es gelang uns mit dieser Studie den Effekt des Alterns auf funktionelle Deaktivierung im somatosensorischen System im Zusammenhang mit ipsilateralen NBR und neuronaler Inhibition während unilateraler sensorischer Stimulation darzustellen. Dabei wählten wir ein jüngeres und älteres Probanden-Kollektiv aus, um mögliche potenzielle Alterungsprozesse zu beobachten und nachvollziehen zu können. Aus diesem Grunde nahmen wir Messungen von positiven und negativen BOLDAntworten im somatosensiblen System sowie von weiteren wichtigen Kortexarealen während unilateraler, elektrischer Stimulation des rechten Nervus medianus mit einer Frequenz von $40 \mathrm{~Hz}$ vor. Zusätzlich ermittelten wir die Wahrnehmungs-Reizschwelle CPT des linken Zeigefingers während rechtsseitiger, elektrischer Stimulation des N. medianus als quantitative Messung der sensorischen Funktion. Des Weiteren ließen wir die Probanden aktiv ihre Finger an der Hand, wo wir zuvor die passiven 
Stimulationen durchführten, beim FT gegenüberstellen. Das FT zeigte, dass die älteren Probanden grundsätzlich keine schlechtere hämodynamische Antwort zeigten, sondern mindestens gleichwertig im Vergleich zu den jüngeren (Abbildungen 18 bis 22). Wie zuvor vermutet wurden deutliche Anstiege des BOLD-Signals (PBR) in contralateralen, primären (Abbildung 25) und bilateralen, sekundären, somatosensorischen Arealen (Abbildung 27) bei den jüngeren und älteren Probanden beobachtet, wohingegen ein Abfall des BOLD-Signals (NBR) im ipsilateralen, primären, somatosensorischen Kortex (Abbildung 26) bei der elektrischen N. medianus-Stimulation stärker hauptsächlich bei den jüngeren Probanden auftrat. Diese NBR korrelierten im Gegensatz zu den älteren Probanden sehr eng mit einem Anstieg der CPT des contralateralen, linken und unstimulierten Zeige-Fingers (Abbildungen 43 und 44). Diese Feststellung unterstützt die These, dass die ipsilaterale NBR eine funktionelle und effektive Inhibition im somatosensiblen System widerspiegelt, welche im höheren Lebensalter zum Großteil verloren geht. Aktiv motorisch induzierte Aufgaben per FT zeigten, dass die älteren Probanden im Vergleich zu den jüngeren nicht unbedingt hämodynamisch weniger, sondern zum Teil deutlicher (Abbildungen 20 und 21), ausbreitender und undifferenzierter Kortexareale mit teilweise höheren Maximalwerten aktivieren. Es erfolgt ein Wechsel von lateralisierten, inhibierten und differenzierten Aktivitäten bei den jüngeren Probanden in hauptsächlich primären und sekundären sensomotorischen Kortexarealen zu einer kompensatorischen Rekrutierung von zusätzlichen Kortexarealen mit einer Verminderung an Inhibition im höheren Lebensalter, um unter kontrollierten Stimulationen möglicherweise das gleiche Outcome wie im jüngeren Alter zu gewährleisten.

Auf Grund unserer Ergebnisse und denen von vorhergegangenen Studien stellen wir die These auf, dass es im höheren Alter bei elektrischer N. medianus-Stimulation zu einer verminderten transkallosalen Inhibition von ipsilateralen sensomotorischen Kortexarealen kommt. Dies deutet darauf hin, dass höheres Alter eine wesentliche Rolle bei Verarbeitungsprozessen im menschlichen Kortex in Bezug auf verschiedene periphere Stimuli spielen kann und bei der Interpretation von fMRTDaten mit BOLD-Signalen vermehrt berücksichtigt werden sollte, insbesondere bei solchen Studien, bei denen eine vermehrte Aktivierung von Kortexarealen bei älteren und Deaktivierung bei jüngeren Probanden festgestellt werden. 


\section{6.) Literaturverzeichnis}

Arthurs OJ, Johansen-Berg H, Matthews PM, Boniface SJ (2004): Attention differentially modulates the coupling of $\mathrm{FMRI} B O L D$ and evoked potential signal amplitudes in the human somatosensory cortex. Exp Brain Res 157, 269-274

Arthurs OJ, Donovan T, Spiegelhalter JD, Pickard JD, Boniface SJ (2007): Intracortically distributed neurovaskular coupling relationships within and between human somatosensory cotices. Cerebral Cortex $\underline{17}$, 661-668

Bandettini PA, Wong EC, Hinks RS, Tikofsky RS, and Hyde JS (1992): Time course EPI of human brain function during task activation. Magn Reson Med 25, 390-397

Baudewig J, Kunkel M, Paulus W: Distribution of cortical activity in motor and sensory tasks focusing on the median nerve: a fMRI study, Vol 1; Proceedings of the ISMRM, Vancouver 1997, 702

Benninghoff A: Anatomie, 13./14. (Bd. 3) und 15. Aufl. (Bd. 2); hrsg. v. Drenckhahn D und Zenker W; Urban und Schwarzenberg, München - Wien - Baltimore 1994

Blankenburg F, Taskin B, Ruben J, Moosmann M, Ritter P, Curio G, Villringer A (2003): Imperceptible stimuli and sensory processing impediment. Science 2999, 1864

Brodmann K: Vergleichende Lokalisationslehre der Großhirnrinde (In ihren Prinzipien dargestellt aufgrund des Zellenbaus); hrsg. v. Barth JA; Leipzig 1909

Büchert M, Schreiber A, Grimm C, Kristeva-Feige R, Otte M, Huppertz HJ, Hennih J, Mergner T, Lücking $\mathrm{CH}$ : Comparison of $\mathrm{fMRI}$ and high resolution EEG imaging with electric sensomotoric stimulation of the median nerve, Vol 1; Proceedings of the ISMRM, Vancouver 1997, 703

Cabeza R (2002): Hemispheric asymmetry reduction in old adults: the HAROLD model. Psychol Aging 17, 85-100 
Cabeza R, Grady CL, Nyberg L, Mclntosh AR, Tulving E, Kapur S, Jennings JM, Houle S, Craik FI (1997): Age-related differences in neural activity during memory encoding and retrieval: a positron emission tomography study. J Neurosci $\underline{17}, 391-$ 400

Chen CC, Tyler CW, Liu CL, Wang YH (2005): Lateral modulation of BOLD activation in unstimulated regions of the human visual cortex. Neuroimage $\underline{24}, 802-809$

Clarey JC, Tweedale R, Calford MB (1996): Interhemispheric modulation of somatosensory receptive fields: evidence for plasticity in primary somatosensory cortex. Cereb Cortex $\underline{6}, 196-206$

Cole KJ, Rotella DL, Harper JG (1999): Mechanisms for age-related changes of fingertip forces during precision gripping and lifting in adults. J Neurosci $\underline{19}$, 32383247

Czisch M, Wehrle R, Kaufmann C, Wetter TC, Holsboer F, Pollmacher T, Auer DP (2004): Functional MRI during sleep: BOLD signal decreases and their electrophysiological correlates. Eur J Neurosci $\underline{20}$, 566-574

Davey NJ, Nowicky AV, Zaman R (2001): Somatopy of perceptual threshold to cutaneous electrical stimulation in man. Exp Physiol 86, 127-130

Davis KD, Wood ML, Crawley AP, Mikulis DJ (1995): fMRI of human somatosensory and cingulate cortex during painful electrical nerve stimulation. NeuroReport $\underline{7}, 321-$ 325

Deisz RA, Prince DA (1989): Frequency-dependent depression of inhibition in guinea-pig neocortex in vitro by GABAB receptor feed-back on GABA release. J Physiol Lond $\underline{412}, 513-541$

Delbono 0 (2003): Neural control of aging skeletal muscle. Aging Cell $\underline{2}, 21-29$ 
D'Esposito M, Zarahn E, Aguirre GK, Rypma B (1999): The effect of normal aging on the coupling of neural activity to the bold hemodynamic response. Neuroimage 10, 6-14

Dorfman LJ, Bosley TM (1979): Age-related changes in peripheral and central nerve conduction in man. Neurology $\underline{29}$, 38-44

Dutta C, Hadley EC, Lexell J (1997): Sarcopenia and physical performance in old age: overview. Muscle Nerve Suppl $\underline{5}, 5-9$

Fabri M, Polonara G, Del Pesce M, Quattrini A, Salvolini U, Manzoni T (2001): Posterior corpus callosum and interhemispheric transfer of somatosensory information: an fMRI and neuropsychological study of a partially callosotomized patient. J Cogn Neurosci 13, 1071-1079

Franceschini MA, Fantini S, Thompson JH, Culver JP, Boas DA (2003): Hemodynamic evoked response of the sensorimotor cortex measured noninvasively with near-infrared optical imaging. Psychophysiology $\underline{40}, 548-560$

Goebel R, Jansma H: Brain Voyager QX, Getting Started Guide, Version 2.0; Brain Innovation B.V., Maastricht 2004, 5-97

Grefkes C, Geyer S, Schormann T, Roland P, Zilles K (2001): Human somatosensory area 2: observer-independent cytoarchitectonic mapping, interindividual variability, and population map. Neurolmage $\underline{14}$, 617-631

Haug H, Eggers R (1991): Morphometry of the human cortex cerebri and corpus striatum during aging. Neurobiol Aging 12, 336-338

Hlushchuk Y und Hari R (2006): Transient suppression of ipsilateral primary somatosensory cortex during tactile finger stimulation. J Neurosci $\underline{26}, 5819-5824$ 
Hummel F, Saur R, Lasogga S, Plewnia C, Erb M, Wildgruber D, Grodd W, Gerloff C (2004): To act or not to act. Neural correlates of executive control of learned motor behavior. Neuroimage 23, 1391-1401

Kakigi R (1994): Somatosensory evoked magnetic fields following median nerve stimulation. Neurosci Res $\underline{20}$, 165-174

Kampe KK, Jones RA, Auer DP (2000): Frequency dependence of the functional MRI response after electrical median nerve stimulation. Hum Brain Mapp $\underline{9}$, 106-114

Kanno A, Nakasato N, Hatanaka K, Yoshimoto T (2003): Ipsilateral area 3b responses to median nerve somatosensory stimulation. Neuolmage $\underline{18}, 169-177$

Kaplan FS, Nixon JE, Reitz M, Rindfleish L, Tucker J (1985): Age-related changes in proprioception and sensation of joint position. Acta Orthop Scand $\underline{56}, 72-74$

Kuhl DE, Metter EJ, Riege WH, Phelps ME (1982): Effects of human aging on patterns of local cerebral glucose utilization determined by the [18F]fluorodeoxyglucose method. J Cereb Blood Flow Metab 2 2, 163-171

Kwong KK, Belliveau JW, Chesler DA, Goldberg IE, Weisskoff RM, Poncelet BP, Kennedy DN, Hoppel BE, Cohen MS, Turner R (1992): Dynamic magnetic resonance imaging of human brain activity during primary sensory stimulation. Proc Natl Acad Sci USA $\underline{89}$, 5675-5679

Leenders KL, Perani D, Lammertsma AA, Heather JD, Buckingham P, Healy MJ, Gibbs JM, Wise RJ, Hatazawa J, Herold S (1990): Cerebral blood flow, blood volume and oxygen utilization. Normal values and effect of age. Brain $\underline{113}, 27-47$

Lexell J (1997): Evidence for nervous system degeneration with advancing age. J Nutr $\underline{127}, 1011-1013$ 
Logan JM, Sanders AL, Snyder AZ, Morris JC, Buckner RL (2002): Underrecruitment and nonselective recruitment: dissociable neural mechanisms associated with aging. Neuron $\underline{33}, 827-840$

Logothetis NK, Wandell BA (2004): Interpreting the BOLD-Signal. Annu Rev Physiol $\underline{66}, 735-769$

Logothetis NK, Pauls J, Augath M, Trinath T, Oeltermann A (2001): Neurophysiological investigation of the basis of the fMRI signal. Nature $\underline{412}, 150-157$

Love RJ, Webb WG: Neurology for the Speech-Language, in: Pathologist; hrsg. von Butterworth-Heinemann; $3^{\text {rd }}$ Edition; Boston 1999, 19

Madden DJ, Gottlob L, Denny L, Turkington T, Provenzale J, Hawk T, Coleman R (1999). Aging and recognition memory: Changes in regional cerebral blood flow associated with components of reaction time distributions. J Cogn Neurosci $\underline{11}$, 511520

Madden DJ, Whiting WL, Huettel SA, White LE, MacFall JR, Provenzale JM (2004): Diffusion tensor imaging of adult age differences in cerebral white matter: relation to response time. Neuroimage $\underline{21}, 1174-1181$

Marchal G, Rioux P, Petit-Taboue MC, Sette G, Travere JM, Le Poec C, Courtheoux P, Derlon JM, Baron JC (1992): Regional cerebral oxygen consumption, blood flow, and blood volume in healthy human aging. Arch Neurol $\underline{49}, 1013-1020$

Mattay VS, Callicott JH, Bertolino A, Santha AK, Tallent KA, Goldberg TE, Frank JA, Weinberger DR (1997): Abnormal functional lateralization of the sensorimotor cortex in patients with schizophrenia. Neuroreport $\underline{8}$, 2977-2984

Mattay VS, Fera F, Tessitore A, Hariri AR, Das S, Callicott JH, Weinberger DR (2002): Neurophysiological correlates of age-related changes in human motor function. Neurology $\underline{58}, 630-635$ 
MR-Forschung: interdisziplinäre Forschungsgruppe der Humanmedizin der GeorgAugust-Universität Göttingen des Bereichs Neurologie und Psychiatrie und des MaxPlanck-Instituts für biophysikalische Chemie in Göttingen, Fragebogen der MRUntersuchungen, Fassung 3/2006, Göttingen 2006

Newton JM, Sunderland A, Gowland PA (2005): fMRI signal decreases in ipsilateral primary motor cortex during unilateral hand movements are related to duration and side of movement. Neuroimage 24, 1080-1087

Nihashi T, Naganawa S, Sato C, Kawai H, Nakamura T, Fukatsu H, Ishigaki T, Aoki I (2005): Contralateral and ipsilateral responses in primary somatosensory cortex following electrical median nerve stimulation - an fMRI study. Clin Neurophysiol $\underline{116}$, 842-848

Ogawa S, Lee TM (1990): Magnetic resonance imaging of blood vessels at high fields: in vivo and in vitro measurements and image simulation. Magn Reson Med $\underline{16}$, $9-18$

Ogawa S, Lee TM, Kay AR, Tank DW (1990). Brain magnetic resonance imaging with contrast dependent on blood oxygenation. Proc Natl Acad Sci USA $\underline{87}, 9868-$ 9872

Ogawa S, Tank DW, Menon R, Ellermann JM, Kim SG, Merkle H, Ugurbil K (1992): Intrinsic signal changes accompanying sensory stimulation: functional brain mapping with magnetic resonance imaging. Proc Natl Acad Sci U S A 89, 5951-5955

O'Sullivan M, Jones DK, Summers PE, Morris RG, Williams SC, Markus HS (2001): Evidence for cortical "disconnection" as a mechanism of age-related cognitive decline. Neurology $\underline{57}, 632-638$

Pluto CP, Chiaia NL, Rhoades RW, Lane RD (2005): Reducing contralateral SI activity reveals hindlimb receptive fields in the SI forelimb-stump representation of neonatally amputated rats. J Neurophysiol $\underline{94}$, 1727-1732 
Poeck K, Hacke W: Neurologie, 11. Auflage; Springer-Verlag, Berlin - Heidelberg New York 2001, 9-11, 625-627

Riecker A, Groschel K, Ackermann H, Steinbrink C, Witte O, Kastrup A (2006): Functional significance of age-related differences in motor activation patterns. Neurolmage $\underline{32}, 1345-1354$

Rosen HJ, Petersen SE, Linenweber MR, Snyder AZ, White DA, Chapman L, Dromerick AW, Fiez JA, Corbetta MD (2000): Neural correlates of recovery from aphasia after damage to left inferior frontal cortex. Neurology $\underline{55}, 1883-1894$

Rother J, Knab R, Hamzei F, Fiehler J, Reichenbach JR, Buchel C, Weiller C (2002): Negative dip in BOLD fMRI is caused by blood flow-oxygen consumption uncoupling in humans. Neuroimage $\underline{15}, 98-102$

Rowe JB, Siebner H, Filipovic SR, Cordivari C, Gerschlager W, Rothwell J, Frackowiak R (2006): Aging is assiciated with contrasting in local distant cortical connectivity in the human motor system. Neurolmage 21, 747-760

Sailer A, Dichgans J, Gerloff C (2000): The influence of normal aging on the cortical processing of a simple motor task. Neurology $\underline{55}, 979-985$

Seyal M, Ro T, Rafal R (1995): Increased sensitivity to ipsilateral cutaneous stimuli following transcranial magnetic stimulation of the parietal lobe. Ann Neurol $\underline{38}, 264-$ 267

Shimoyama I, Ninchoji T, Uemura K (1990): The finger-tapping test. A quantitative analysis. Arch Neurol 47, 681-684

Shmuel A, Yacoub E, Pfeuffer J, Van de Moortele PF, Adriany G, Hu X, Ugurbil K (2002): Sustained negative BOLD, blood flow and oxygen consumption response and its coupling to the positive response in the human brain. Neuron $\underline{36}, 1195-1210$ 
Shmuel A, Augath M, Oeltermann A, Logothetis NK (2006): Negative functional MRI response correlates with decreases in neuronal activity in monkey visual area V1. Nat Neurosci $\underline{9}, 569-577$

Smith AT, Williams AL, Singh KD (2004): Negative BOLD in the visual cortex: evidence against blood stealing. Hum Brain Mapp 21, 213-220

Smith CD, Umberger GH, Manning EL, Slevin JT, Wekstein DR, Schmitt FA, Markesbery WR, Zhang Z, Gerhardt GA, Kryscio RJ (1999): Critical decline in fine motor hand movements in human aging. Neurology $\underline{53}, 1458-1461$

Stefanovic B, Warnking JM, Pike GB (2004): Hemodynamic and metabolic responses to neuronal inhibition. Neuroimage 22, 771-778

Sutherland MT, Tang AC (2006): Reliable detection of bilateral activation in human primary somatosensory cortex by unilateral median nerve stimulation. Neuroimage 33, 1042-1054

Talairach J, Tournoux P: Co-planar stereotaxic atlas of the human brain: 3dimensional proportional system: an approach to cerebral imaging; Thieme Medical Publishers, New York USA, 1988

Tekes A, Mohamed MA, Browner NM, Calhoun VD, Yousem DM (2005): Effect of age on visuomotor functional MR imaging. Acad Radiol $\underline{12}, 739-745$

Thulborn KR, Waterton JC, Matthews PM, Radda GK (1982): Oxygenation dependence of the transverse relaxation time of water protons in whole blood at high field. Biochim Biophys Acta $\underline{714}$, 265-270

Trepel M: Neuroanatomie: Struktur und Funktion, 2. Auflage; Urban und Fischer, München - Stuttgart - Jena - Lübeck - Ulm 1999, 181-231

Van Westen D, Fransson P, Olsrud J, Rosén B, Lundborg G, Larsson EM (2004):

Fingersomatotopy in area 3b: an fMRI-study. BMC Neurosci $\underline{5}, 28$ 
Ward NS, Frackowiak RS (2003): Age-related changes in the neural correlates of motor performance. Brain $\underline{126}, 873-888$

Weinberger D: Schizophrenia as a neurodevelopmental disorder; in: Schizophrenia; edited by Hirsch S, Weinberger D; Blackwells, Oxford 1995, 293-323

Weltärztebund, Deklaration des Weltärztebundes von Helsinki, Ethische Grundsätze für die medizinische Forschung am Menschen, verabschiedet von der Generalversammlung, Helsinki 1964 - Tokio 2004

Yamashita T, Kanaya K, Sekine M, Takebayashi T, Kawaguchi S, Katahira G (2002): A quantitative analysis of sensory function in lumbar radiculopathy using current perception threshold testing. Spine $\underline{27}, 1567-1570$ 


\section{7.) Abkürzungsverzeichnis}

\begin{tabular}{|c|c|}
\hline BA & Brodmann-Areal (brodmann area) \\
\hline BOLD-Effekt & blood-oxygenation-level-dependent-Effekt \\
\hline C5 & zervikales Rückenmarksegment 5 \\
\hline $\mathrm{CBF}$ & lokaler zerebraler Blutfluss (regional cerebral blood flow) \\
\hline CBV & zerebrales Blutvolumen \\
\hline $\mathrm{CMRO}_{2}$ & zerebraler metabolischer Umsatz von Sauerstoff \\
\hline $\mathrm{CO}_{2}$ & Kohlenstoffdioxid \\
\hline CPT & Wahrnehmungsschwelle (current perception threshold) \\
\hline CT & Computertomograph \\
\hline Desoxy-Hb & desoxygeniertes Hämoglobin \\
\hline DTI & Diffusion Tensor Imaging \\
\hline EEG & Elektroenzephalographie \\
\hline EPI & echoplanare Bildgebung (echo planar imaging) \\
\hline FDR & Rate fälschlich entdeckter Voxel (false discovery rate) \\
\hline FLASH & fast low angle shot \\
\hline fMRT & funktionelle Magnetresonanztomographie \\
\hline FT & Finger-Gegenüberstellung (finger tapping) \\
\hline GLM & allgemeines lineares Modell (general linear model) \\
\hline HAROLD & hypothesis of hemispheric asymmetry reduction on older adults \\
\hline $\mathrm{HF}$ & Hochfrequenz \\
\hline HRF & hämodynamische Reizantwort \\
\hline $\mathrm{Hz}$ & Herz \\
\hline IPSP & inhibitorische postsynaptische Potenziale \\
\hline KM & Kontrastmittel \\
\hline MEG & Magnetenzephalographie \\
\hline MI & motorischer Kortex \\
\hline MRT & Magnetresonanztomographie \\
\hline NBR & negative BOLD-Antwort (negative BOLD response) \\
\hline $\mathrm{Ncl}$ & Nucleus \\
\hline NO & Stickstoffmonoxid \\
\hline $\mathrm{O}_{2}$ & Sauerstoff \\
\hline $\mathrm{Oxy}-\mathrm{Hb}$ & oxygeniertes Hämoglobin \\
\hline
\end{tabular}




$\begin{array}{ll}\text { PBR } & \text { positive BOLD-Antwort (positive BOLD response) } \\ \text { PET } & \text { Positronen-Emissions-Tomographie } \\ \text { ROI } & \text { interessierender Bereich (region of interest) } \\ \text { SD } & \text { Standardabweichung (standard deviation) } \\ \text { SI } & \text { primärer somatosensorischer Kortex } \\ \text { SII } & \text { sekundärer somatosensorischer Kortex } \\ \text { T2 } & \text { T2-Wichtung im MRT } \\ \text { TAL } & \text { Talairach } \\ \text { TE } & \text { Echozeit (time of echo) } \\ \text { Th1 } & \text { thorakales Rückenmarkssegment 1 } \\ \text { TI } & \text { Umkehr-Zeit (time of inversion) } \\ \text { TMS } & \text { transkranielle Magnetstimulation } \\ \text { TR } & \text { Repetitionszeit (time of repetition) } \\ \text { V1 } & \text { Visueller Kortex } \\ \text { VPL } & \text { Nucleus ventralis posterolateralis } \\ \text { VPM } & \text { Nucleus ventralis posteromedialis } \\ \text { ZNS } & \text { zentrales Nervensystem }\end{array}$




\section{Danksagung}

Herrn Prof. Dr. med. A. Kastrup danke ich sehr für die Möglichkeit zur Promotion in der Abteilung Neurologie der Medizinischen Fakultät der Georg-August-Universität Göttingen sowie für die wertvollen Anregungen und die Betreuung bei den Messungen und der Ausarbeitung der Dissertation.

Mein besonderer Dank gilt Frau Dr. med. S. Schnaudigel für die engagierte Mitarbeit bei der Auswertung der Arbeit.

Ebenfalls bedanke ich mich sehr bei Herrn Dr. J. Baudewig, Dr. P. Dechent und Frau P. Holz aus der interdisziplinären Forschungsgruppe, MR-Forschung in der Neurologie und Psychiatrie, aus der Humanmedizin der Georg-August-Universität, Göttingen und des Max-Planck-Instituts für biophysikalische Chemie, Göttingen für die Möglichkeit, die fMRT-Technik nutzen zu können und die technische Betreuung bei den Messungen und bei der Auswertung.

Weiterhin danke ich besonders Herrn Cand. med. L. Becker für die sehr gute Zusammenarbeit und Frau G. Ilse für die Organisation von freiwilligen, gesunden und älteren Probanden.

Ein besonderer Dank geht an meine Familie und Freunde, die sich zum Beispiel als Probanden zur Verfügung stellten und mich in dieser Zeit sehr unterstützt haben. 